NASACONTRACTOR REPOR T

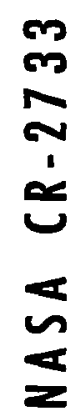

U.S.A. LOAN COPY: FETUEN TO

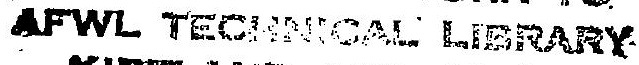
KIRTARE MFE, RE,

\title{
ACOUSTIC RADIATION AND SURFACE PRESSURE CHARACTERISTICS OF AN AIRFOIL DUE TO INCIDENT TURBULENCE
}

Robert W. Paterson and Roy K. Amiet

Prepared by

UNITED TECHNOLOGIES. REȘEARCH CENTER

East Hartford, Conn. 06108

for Langley Researcb Center

and U.S. Army Air Mobility RED Laboratory

NATIONAL aERONAUtics AND SPACE ADMINISTRATION - WASHINGTON, D. C. - SEPTEMBER 1976

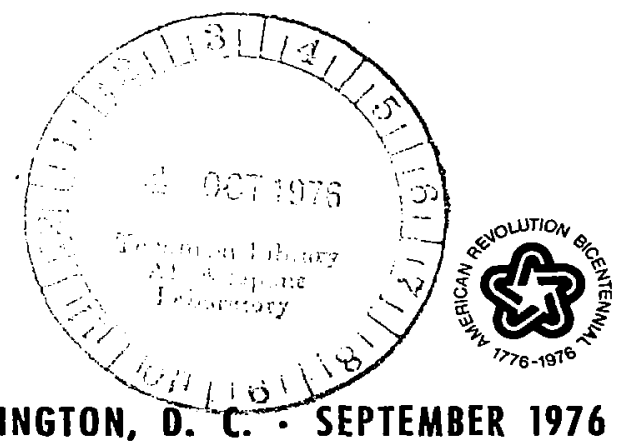




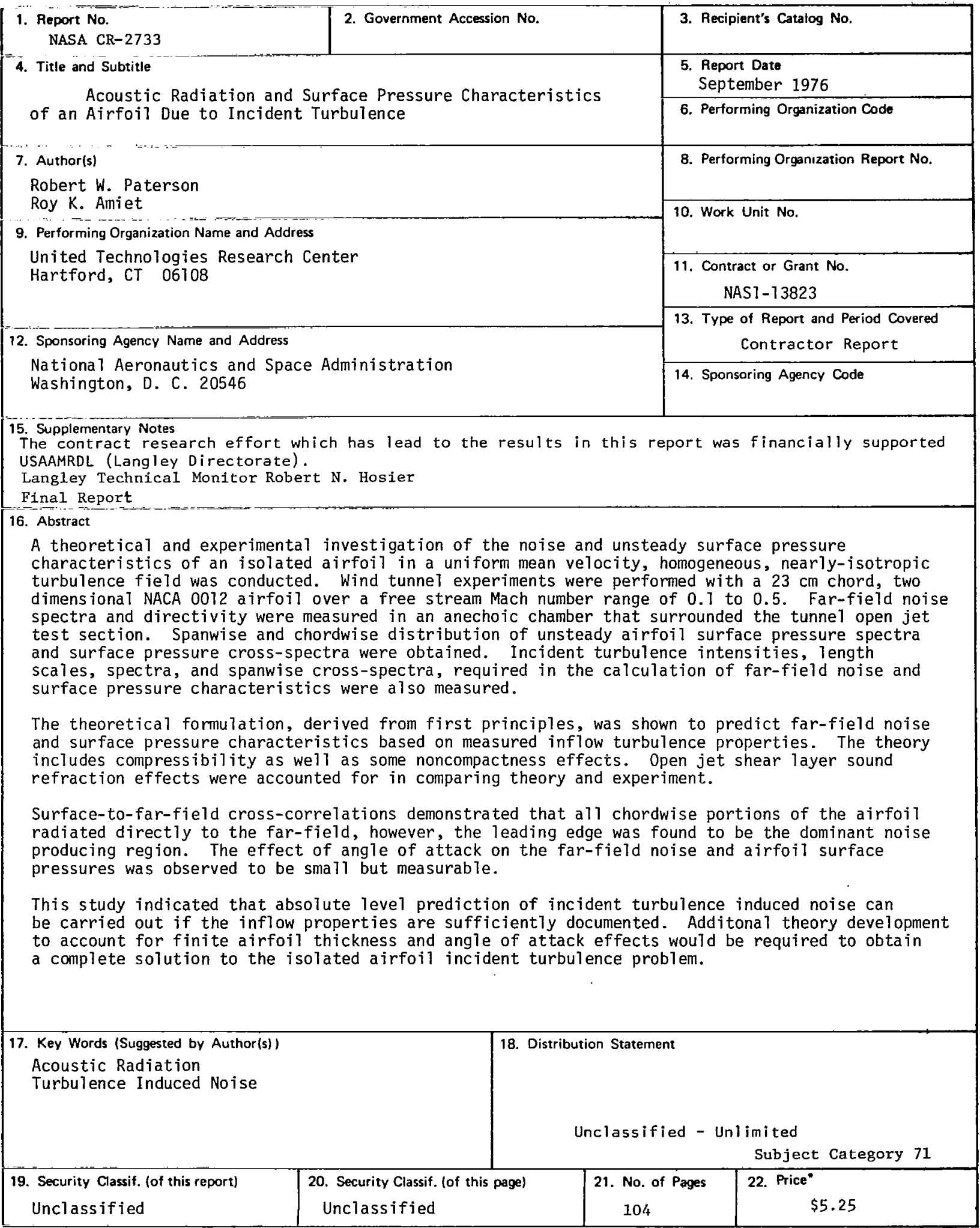

* For sale by the National Technical Information Service, Springfield, Virginia 22161 
TABTE OF CONTENTS

$\underline{\text { Page }}$

SUMMARY . . . . . . . . . . . . . . . . . . 1

INTRODUCTION . . . . . . . . . . . . . . . . . 2

LIST OF SYMBOLS . . . . . . . . . . . . . . . . 4

DESCRIPTION OF THE EXPERIMENT . . . . . . . . . . . . 9

Experimental Arrangement ................ 9

Airfoil Model .................... 11

Instrumentation ................. II

Turbulence Grid Design . . . . . . . . . . . . . 12

Test Program ................... I3

DEFINITION OF THE INCIDENT TURBULENCE FIELD . . . . . . . . . 14

Required Measurements ............... 14

Spanwise Homogeniety ................. . 14

Turbulence Length Scales . . . . . . . . . . . . . 15

Turbulence spectra . . . . . . . . . . . . . . 16

Spanwise Cross-Correlations ............. 16

Spanwise Cross-Spectra .. .............. 16

Summary of Flow Measurement Results . . . . . . . . . 17

FAR FIEID ANALYSES AND RESULTS . . . . . . . . . 18

Far Field Noise Theory ................ 18

Far Field Experimental Results . . . . . . . . . 24

Importance of Incident Turbulence as a Noise Mechanism . . . 27

Approximate Expressions for Far-Field Noise. . . . . . . 28

Far Field Summary . . . . . . . . . . . . 28

SURFACE PRESSURE ANALYSES AND RESULTS . . . . . . . . . . . . 29

Surface Pressure Theory . . . . . . . . . . . 29

Surface Pressure Experimental Results . . . . . . . . 32 
TABIE OF CONTENTS (Cont'd)

Page

ADDITIONAL STUDIES. . . . . . . . . . . . . . . 37

Correlation Studies. . . . . . . . . . . . . . 37

Angle of Attack Effects. . . . . . . . . . . . . . . . 4 I

Prediction of Far-Field Noise from Surface Pressure

Measurements. . . . . . . . . . . . . . 42

CONCLUSIONS . . . . . . . . . . . . . . . . . 47

APPENDIX A. . . . . . . . . . . . . . . . 48

Discission of Previous Investigations. . . . . . . . . . 48

APPENDIX B:.......................5I

Cross-PSD Determination. . . . . . . . . . . . 51

APPENDIX C. . . . . . . . . . . . . . . . . 54

Hot Wire Measurements. . . . . . . . . . . . 54

APPENDIX D. . . . . . . . . . . . . . . . . . . 56

Derivation of the von Kármán Turbulence Functions. . . . . . 56

APPENDIX E. . . . . . . . . . . . . . . . . . 58

Approximate Expressions for Far-Field Noise. . . . . . . . 58

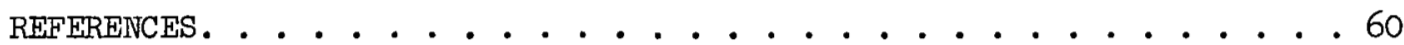

TABLES. . . . . . . . . . . . . . . . 64

FIGURES . . . . . . . . . . . . . . . 66 
Acoustic Radiation and Surface Pressure Characteristics

of An Airfoil Due to Incident Turbulence

By

Robert W. Paterson and Roy K. Amiet

SUMMARY

A theoretical and experimental investigation of the noise and unsteady surface pressure characteristics of an isolated airfoil in a uniform mean velocity, homogeneous, nearly isotropic turbulence field was conducted. Experiments were performed with a $23 \mathrm{~cm}$ chord, two-dimensional NACA 0012 airfoil in the United Technologies Research Center (UTRC) Acoustic Research. Tunnel over a free stream Mach number range of 0.1 to 0.5 . Far-field noise spectra and directivity were measured in an anechoic chamber that surrounded the tunnel open jet test section. Spanwise and chordwise distributions of unsteady airfoil surface pressure spectra and surface pressure cross-spectra were obtained. Incident turbulence intensities, length scales, spectra and spanwise crossspectra required in the calculation of far-field noise and surface pressure characteristics were measured.

When applied to predict far-field noise and surface pressure characteristics from measured inflow turbulence properties the theory showed good agreement with measurement over the dominant frequency range for all Mach numbers investigated. The theoretical formulation represents a first-principles solution providing absolute level prediction without recourse to empirical or adjustable constants. It includes compressibility as well as source noncompactness effects. Open-jet shear layer sound refraction effects were accounted for in comparing theory and experiment. Comparison of theory and directivity data confirms the validity of applied refraction propagation corrections.

Surface-to-far-field cross-correlations demonstrated that all chordwise portions of the airfoil radiated directly to the far-field as opposed to the results which are obtained with other isolated airfoil noise mechanisms such as discrete frequency vortex shedding and stalled airfoil flow. The leading edge, however, was found to be the dominant noise producing region of the airfoil. The effect of angle of attack on far-field noise and surface pressures was observed to be small but measureable. The interaction of incident turbulence of approximately 4 percent intensity with an airfoil was found to be a broadband noise source of high intensity relative to other noise sources such as the turbulent boundary layer and stalled airfoil flow investigated previously. The study indicated that absolute level prediction 
of incident turbulence noise can be carried out if the inflow turbulence properties are sufficiently documented. Additional theory development to account for finite airfoil thickness and angle of attack effects would be required to obtain a complete solution to the isolated airfoil incident turbulence problem.

\section{INTRODUCTION}

Acoustic radiation by an airfoil due to incident turbulence is an important phenomenon since it is both an effective noise generating mechanism and its occurance is widespread. An indication of its effectiveness in creating sound is apparent from the simple experiment of placing a blade in the potential core of a free jet and observing the significant increase in noise as the blade is moved downstream into turbulent regions of greater spanwise extent. This mechanism occurs to some extent in all propulsion devices such as helicopter rotors, propellers and turbofan engines and may also be important relative to airframe noise generation.

For turbofan engine strut and stator vane noise as well as airframe noise, isolated airfoil results are directly applicable to the prediction of broadband noise generation. In rotating devices, isolated airfoil results can be applied in a stripwise manner to predict broadband noise. While quasi-tonal noise generation due to interaction of turbulence with rotating blades is also an important problem, it is more complex. The geometry is more complicated and adequate definition of turbulence inflow statistics, particularly in the problem of ingestion of atmospheric turbulence by a rotor (helicopter or fan) or propeller, is difficult.

The present study was undertaken to treat the stationary, isolated airfoil problem in a rigorous theoretical manner and with an experimental configuration capable of direct assessment of theory. If theory could be validated for this geometry, the basis would be established for extension to more complex cases such as rotating blades.

While the theoretical foundation for studying this problem extends to the early unsteady airfoil theory development of von Kármán and Sears (reference 1) and the acoustic formulation of curle (reference 2), Sharland (reference 3) in 1964 was the first to experimentally assess and attempt to predict incident turbulence noise. He measured the noise of a small airfoil placed in a turbulent jet and with a relatively simple theory estimated the noise at one directivity angle showing reasonable agreement with data. Many approximations and some empiricism were involved. Sharland's study as well as that of subsequent 
investigators are reviewed in detail in Appendix A. With the exception of the recent study of Fink (reference 4), all of these noise investigations were subject to some uncertainty because the mean velocity and incident turbulence statistics were not uniform across the airfoil span and the turbulence statistics required for rigorous prediction of noise were not measured. Fink's study employed sideplates to provide uniform spanwise conditions. The incident turbulence was documented although spanwise cross-spectrum, required in a complete theory, was not obtained.

In addition to these studies directed toward measurement of incident turbulence far-field noise as a function of the relevant parameters, Clark and Ribner (réference 5) and Siddon (reference 6) conducted correlation studies directed toward increased understanding of the fundamental noise generation process. In addition to reviewing previous experimental noise investigations, Appendix A discusses existing theoretical formulations of the incident turbulence problem.

In the present experiment, the simplest geometry of an isolated airfoil in a uniform, spanwise homogeneous, nearly isotropic turbulent field was employed. A large airfoil model ( $23 \mathrm{~cm}$ chord) permitted investigation of the important regime in which the simplifying assumption of acoustical compactness did not apply. All of the turbulence statistical parameters affecting radiated noise were measured. Far-field directivity and spectra as well as airfoil unsteady surface pressure spectra and correlations were obtained. Surface-to-far-field correlations were performed to assist understanding of the noise generation process. Theory was applied to predict far-field noise spectra and directivity as well as surface spectra and cross-spectra. Predictions of theory were compared to experimental measurements. 


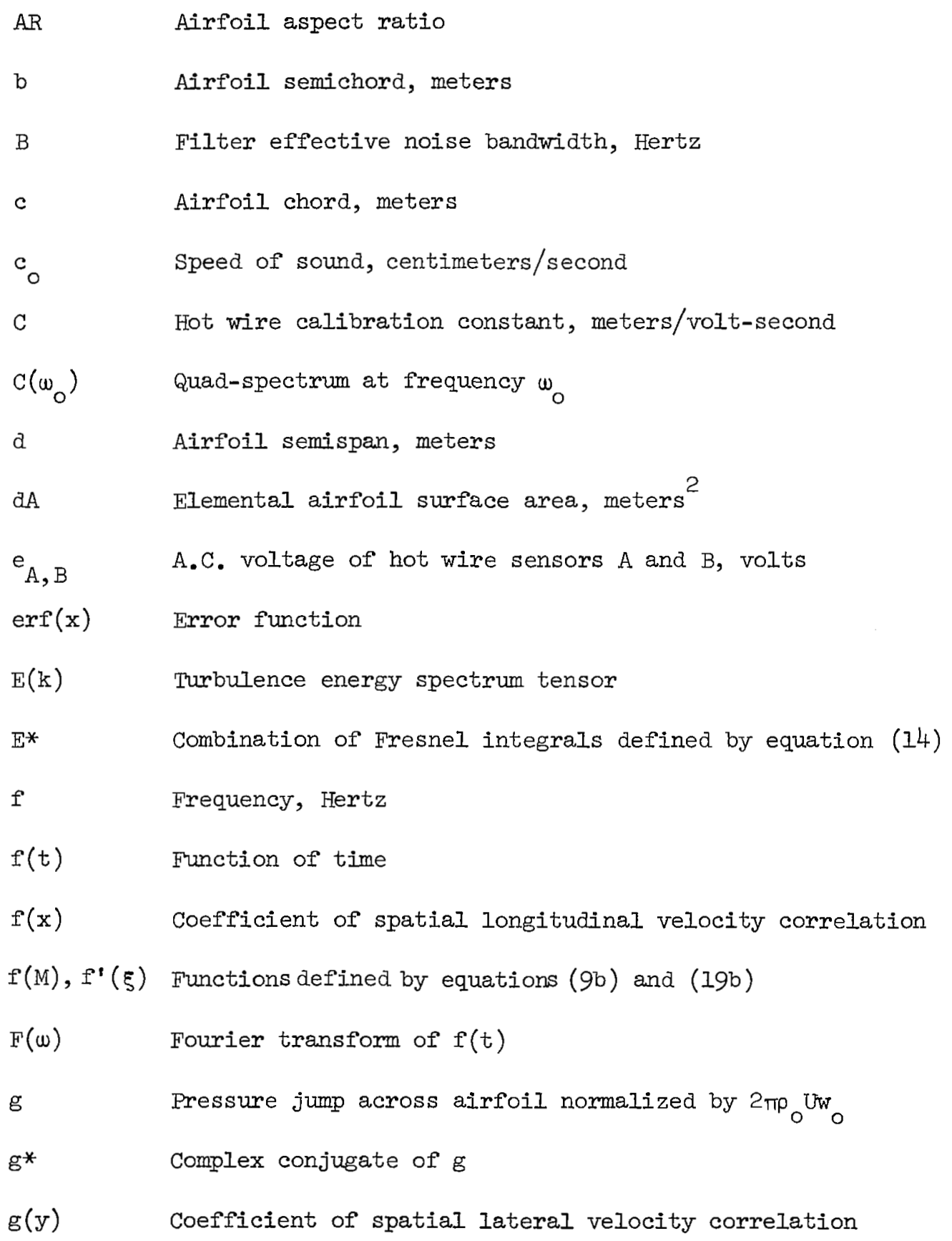


LIST OF SYMBOLS (Cont'd)

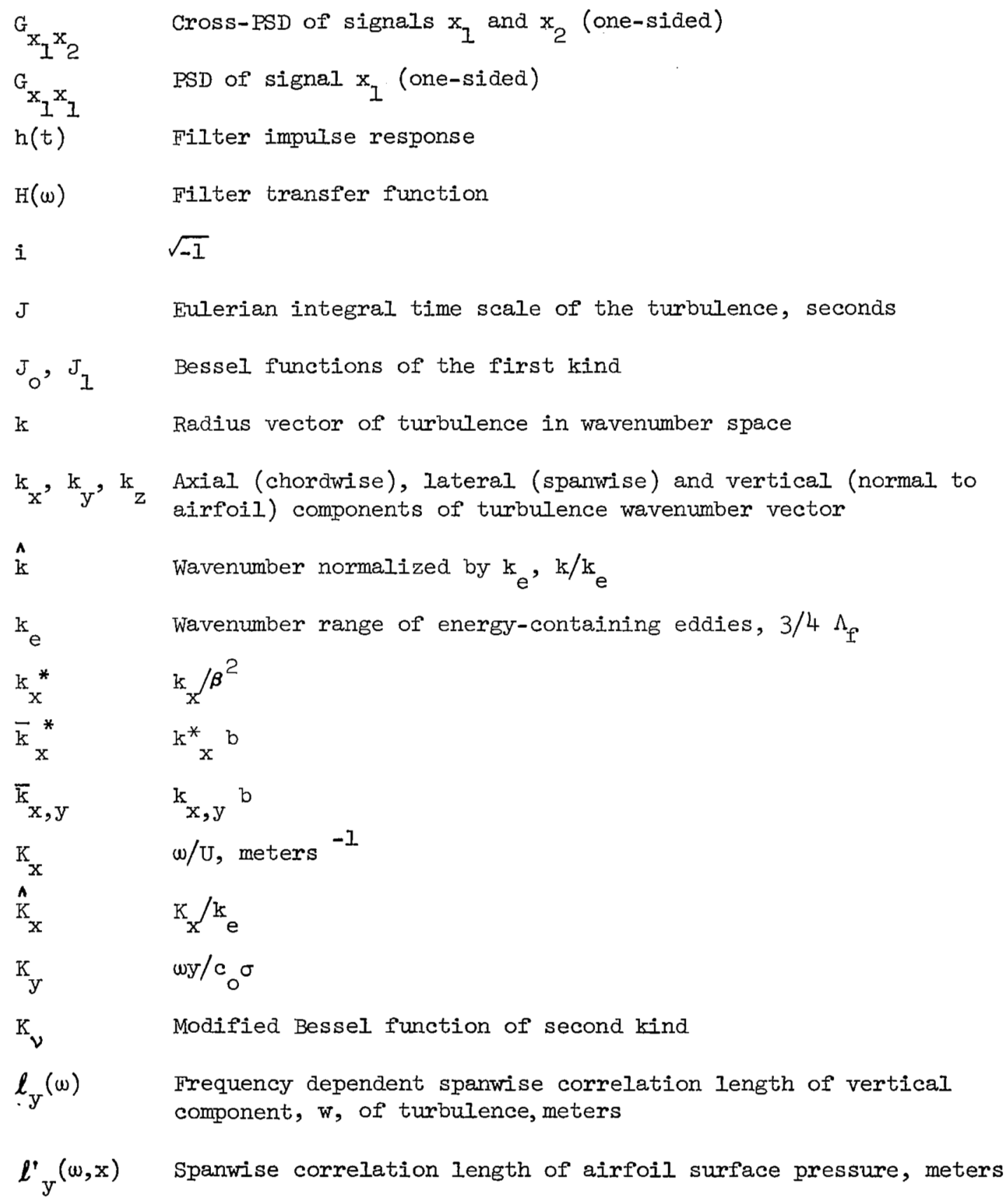




\section{LIST OF SYMBOIS (Cont'd)}

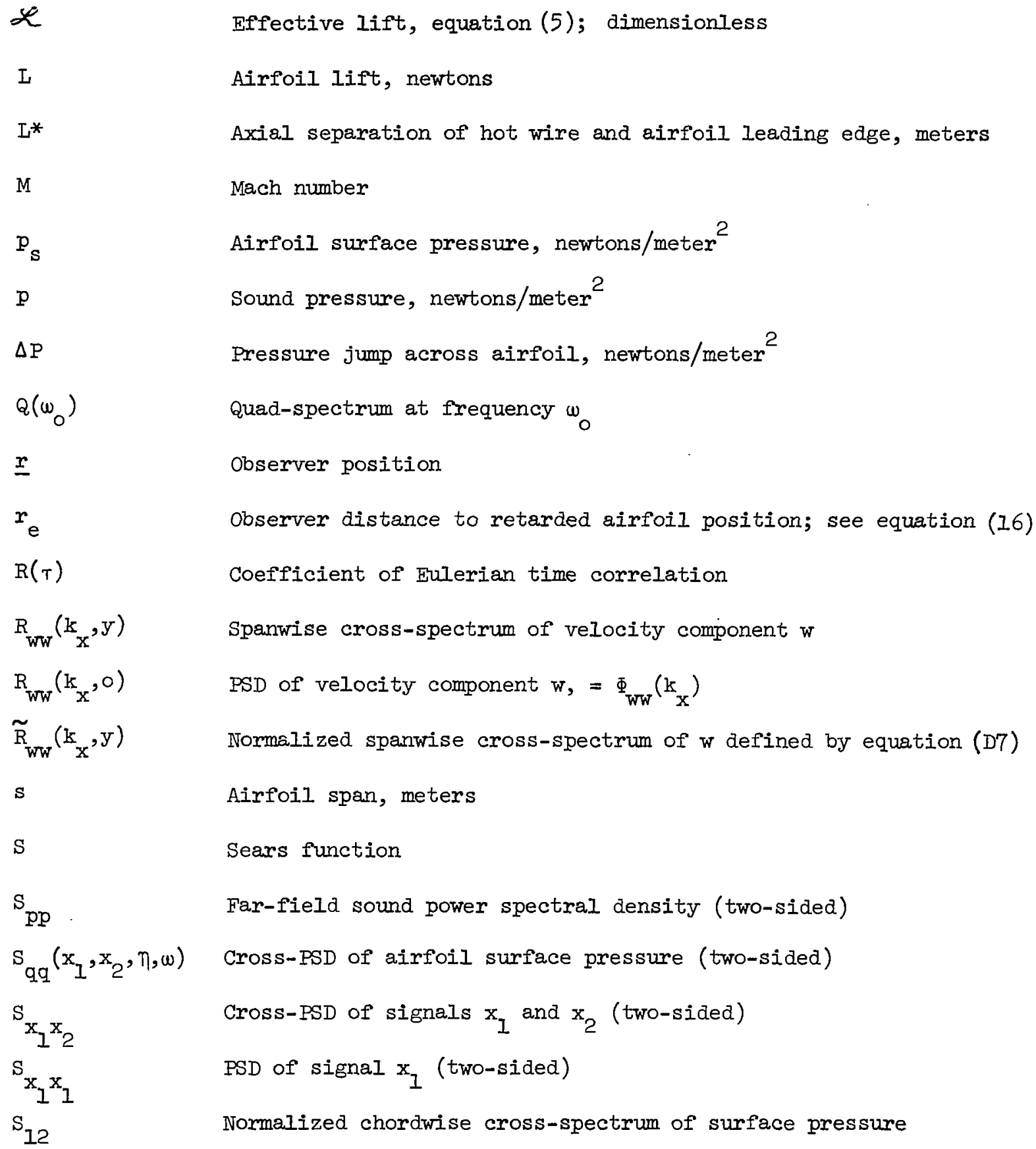


IIST OF SYMBOIS (Cont'd)

$t$

$t_{A}$

$\mathrm{T}$

u

$u^{2}$

$\mathrm{U}$

$\mathrm{U}_{\text {eff }}$

W

$\mathrm{w}_{\mathrm{g}}$

wo

$x$

$\overline{\mathrm{x}}$

y

$\mathrm{y}^{*}$

$\hat{y} *$

z

$z(\tau)$

$\alpha$

$\beta^{2}$

$\Gamma$

$\eta$

$\eta_{0}$

Time, seconds

Airfoil maximum thickness, meters

Horlock function, defined by equation (26)

Axial turbulent velocity component, meters/second

Mean square value of $u$

Mean velocity in axial direction, meters/second

Effective velocity incident on inclined hot wire, meters/second

Vertical (normal to airfoil) turbulent velocity component, meters/second

Gust velocity normal to airfoil, meters/second

Magnitude of gust velocity, meters/second

Axial, Cartesian coordinate

$\mathrm{x} / \mathrm{b}$

Lateral (spanwise) Cartesian coordinate

$y k e^{\sqrt{1+\hat{k}_{x}^{2}}}$

$(4 / 3) y^{*}$

Vertical (normal to airfoil) Cartesian coordinate

Correlator output

Airfoil angle of attack, degrees

$1-M^{2}$

Gamma function

Spanwise separation distance, meters

Dummy variable 


\section{LIST OF SYMBOLS (Cont'd)}

$\theta$

$\theta_{\mathrm{e}}$

$\Theta_{1,2}$

$\lambda$

$\Lambda_{\mathrm{f}}$

$\Lambda_{\mathrm{g}}$

$\mu$

$\xi$

$\xi_{0}$

po

$\sigma^{2}$

T

${ }^{\top} I$

$T_{2}$

$\emptyset_{M}$

$\Phi_{u u}\left(k_{x}\right)$

$\Phi_{W W}\left(k_{x}, k_{y}, k_{z}\right)$

$\Phi_{W W}\left(k_{x}, k_{y}\right)$

$\Phi_{w W}\left(k_{x}\right)$

$\omega$

$w_{0}$
Directivity angle in $\mathrm{x}, \mathrm{z}$ plane measured relative to downstream axis, $\theta=180-\phi$, degrees

Retarded angle, defined by equation (16), degrees

Functions defined by equation (15c)

Acoustic wavelength, meters

Longitudinal space integral scale of turbulence, meters

Lateral space integral scale of turbulence, meters

$\mathrm{MK}_{\mathrm{x}} \mathrm{b} / \beta^{2}$

Dummy variable

Dummy variable

Free stream density, grams/centimeter ${ }^{3}$

$x^{2}+\beta^{2}\left(y^{2}+z^{2}\right)$

Time delay, microseconds

$I^{*} / \mathrm{U}$, microseconds

$r / c_{0}$, microseconds

Directivity angle in $\mathrm{x}, \mathrm{z}$ plane measured relative to upstream axis, $\phi=180-\theta$, degrees

One wavenumber spectrum (PSD) of $u$

Three wavenumber spectrum of vertical velocity component, w

Two wavenumber spectrum of $\mathrm{w}$

One wavenumber spectrum (PSD) of w

Circular frequency, radians/second

Filter center frequency, radians/second 


\section{DESCRIPTION OF THE EXXPERTMENT}

\section{Experimental Arrangement}

This study was conducted in the UTRC Acoustic Research Tunnel. The tunnel, shown schematically in figure 1 and described in detail in references 7 and 8 , is a controlled turbulence level, open-jet, open-circuit wind tunnel designed specifically for aerodynamic noise research. Models placed within the test section generate noise which propagates through the open-jet velocity field to microphones located in the quiescent region of the sealed chamber that surrounds the open-jet test section. This chamber is lined with $0.3 \mathrm{~m}$ depth, two-dimensional fiber glass wedges and has been demonstrated through acoustic calibrations to provide an anechoic test environment for broadband noise at sound frequencies above $200 \mathrm{~Hz}$ (reference 7). Since isolated airfoil, incident turbulence noise is broadband in nature, microphones located in the geometric and acoustic far-field of the model detect airfoil far-field noise under reflection-free conditions above $200 \mathrm{~Hz}$. Propagation of airfoil noise through the open-jet shear layer causes refraction of sound wavefronts which must be accounted for in data interpretation.

In the absence of turbulence generators in the tunnel inlet, the combined effect of an inlet honeycomb, five screens and a 16.5 area ratio inlet contraction is to provide a test section total turbulence level of approximately 0.2 percent and a mean velocity spatially uniform to within 0.5 percent (reference 7). The test section flow is collected at the diffuser entrance by a jet collector lined with fiber glass to reduce sound reflection. An extensive noise muffling section located downstream of the diffuser reduces tunnel fan noise propagation to the test section. Measurements indicate that tunnel background noise is dominated by impingement of the open-jet flow on the jet collector for frequencies above which the chamber is anechoic. A $1500 \mathrm{hp}$ variable speed centrifugal fan drives the tunnel. The test section velocity is temporally steady thereby producing a statistically stationary airfoil noise signal.

Figure 2 displays the anechoic chamber test section arrangement employed in this study. The test airfoil was mounted horizontally between two vertical sideplates. The airfoil support permitted angle of attack variations. The sideplates extended $1.23 \mathrm{~m}$ downstream from the $0.53 \mathrm{~m}$ (wide) by $0.79 \mathrm{~m}$ (high) inlet. The sideplates provided two-dimensional flow conditions and eliminated the need to extend the airfoil through the open jet shear layer that would have existed in their absence. Noise generated by impingement of the highly turbulent shear layer on the airfoil would have produced significant extraneous noise that could not have been subtracted from the test 
data in a rigorous manner. This extraneous noise has existed in previous studies such as those described in reference 9. The sideplate turbulent boundary layer is thin (order $0.18 \mathrm{~cm}$ displacement thickness) and is not a significant source of noise since tunnel background noise with and without sideplates is approximately the same.

Figure 2(a) shows a side elevation of the anechoic chamber. The leading edge of the airfoil was located $0.24 \mathrm{~m}$ downstream of the inlet. Far-field microphones were located on a $2.25 \mathrm{~m}$ radius arc relative to the airfoil centerline in a vertical plane on the tunnel centerline. A total of six far-field microphones were employed with angles relative to the upstream direction of $70,90,105,120,130$ and $140 \mathrm{deg}$. Line-of-sight interference between the airfoil and far-field microphones by the tunnel inlet occurs at angles of 50 deg and less. The forward quadrant angle of 70 deg was considered the minimum achievable for which inlet reflection and diffraction effects would be expected to be negligible. To provide maximum test section length, the jet collector was located on the aft chamber wall. This yielded a $3.3 \mathrm{~m}$ long test section with unobstructed sound propagation to the aft quadrant microphones. The presence of acousticaliy untreated sideplates, however, raises the question of data validity due to potential reflection and diffraction effects. At frequencies greater than about $700 \mathrm{~Hz}$, such that the sound wavelength is smaller than the sideplate separation distance $(0.53 \mathrm{~m})$, ray acoustics arguments are applicable. Assuming specular reflection from the sideplates, the reflection effect as viewed by the far-field microphone is one of an effective increase in airfoil span by an amount equal to the mirror image of the airfoil in the sideplate. Since the angle between the reflected ray that propagates to the far-field microphone and the sideplate is small, the overall reflection effect would also be expected to be small. At lower frequencies such arguments are not applicable and some uncertainty exists. Based on the observed good agreement between theoretical prediction and experiment at low frequency as well as frequencies above $700 \mathrm{~Hz}$, as discussed in the section entitled "Far-Field Experimental Results", sideplate effects were not considered to be important. While the sideplates introduced some uncertainty, the advantages accruing from their use in providing two-dimensional flow conditions and eliminating the need to extend the airfoil or supports through a turbulent shear layer, outweighed the potential disadvantages.

Figure 2(b) shows an end view of the test arrangement looking upstream. The turbulence grid seen in this view was employed to generate turbulence incident on the test airfoil. The grid was located $1.22 \mathrm{~m}$ upstream of the inlet nozzle lip in a $1.07 \mathrm{~m}$ dia section of the inlet contraction. Between the grid and nozzle lip the inlet changed in cross-section from circular to rectangular and contracted in area by a factor of 2.2. The grid design is later discussed in the section "Turbulence Grid Design." 


\section{Airfoil Model}

The test airfoil shown in figure 2 was a $0.23 \mathrm{~m}$ chord, $0.53 \mathrm{~m}$ span, NACA 0012 airfoil. The model was instmmented with an array of four fixed and one moveable $0.635 \mathrm{~cm}$ dia flush-mounted condenser microphones on the airfoil upper surface. Four fixed microphones were located at one-third span and $15,38,50$ and 70 percent chord, respectively. A microphone at 30 percent chord was housed in a slider capable of traverse over the complete span of the airfoil. This array permitted measurement of the local fluctuating surface pressure in both the span and chord directions. The microphone protective grids were removed and the diaphragms optically aligned with the airfoil surface to minimize interference with surface pressure measurements by wakes from upstream microphones. This microphone arrangement had been successfully employed in two previous investigations of isolated airfoil noise (references 10 and 11 ).

\section{Instrumentation}

Far-field and unsteady surface pressures were measured with $0.635 \mathrm{~cm}$ dia condenser microphones with protective grids removed. The frequency response of these microphones was flat for the range relevant to this study (200 to $5000 \mathrm{~Hz}$ ). Since the correlation lengths associated with turbulenceinduced surface pressure fluctuations were found to be large compared to the microphone diaphragm diameter, correction of data for finite microphone size effects were not required. This differs from wall measurements of boundary layer pressure fluctuations where the ratio of correlation length to transducer diameter is not small and significant corrections are required at high frequency. Atmospheric attenuation of far-field sound was calculated to be negligible for the low frequencies and the small sound propagation distance of the experiment and corrections were not applied.

Spectrum analysis was conducted with a 500 line, narrow bandwidth, realtime spectrum analyzer-ensemble averager. By operating with an averaging time of $25 \mathrm{sec}, 512$ statistical degrees of freedom were associated with the resultant power spectrum estimates. The 80 percent confidence limits corresponding to this value are approximately $\pm 0.3 \mathrm{~dB}$ (reference 12 ).

The spectrum analyzer employs quasi-peak detection resulting in a 1.1 $\mathrm{dB}$ higher response to random than pure tone inputs. A negative amplitude correction of $1.1 \mathrm{~dB}$ was therefore applied to data to account for the difference in response to the pure tone calibration signal and the broadband test data. Operating. with an analysis range of 0 to $10 \mathrm{KHz}$, the effective noise bandwidth of the analyzer filter was measured to be $55.7 \mathrm{~Hz}$. To convert to 
$1 \mathrm{~Hz}$ bandwidth, the amplitude of test data was reduced an additional $17.5 \mathrm{~dB}$. In this report, amplitudes of noise and surface pressure spectra are presented in terms of "spectrum level" which is defined as the sound pressure level in decibels referred to $0.0002 \mu$ bar based on a $1 \mathrm{~Hz}$ bandwidth analysis. For broadband noise such as encountered in this experiment, spectrum level obtained as discussed above is equivalent to power spectral density (PSD).

Correlations were performed with a 100 line, real-time correlation and probability analyzer. To obtain cross-power spectral density (cross-PSD) information, input signals were filtered prior to cross-correlation with bandpass filters tuned to the center frequency for which cross-PSD estimates were required. This method for determining cross-PSD is discussed in Appendix $B$.

Turbulence data were acquired with single and crossed-wire hot wire probes of $0.005 \mathrm{~mm}$ dia operating in conjunction with a four channel, linearized, constant temperature anemometer system. A sum and difference network was employed to determine axial and vertical turbulence velocity component information from crossed-wire probes. The probes were calibrated in the tunnel test section. Hot wire measurements are discussed in more detail in Appendix C.

Tunnel speed was determined by measuring the difference between total pressure in the tunnel inlet (downstream of the tunnel inlet screens but upstream of the turbulence generating grid) and the chamber static pressure. Since the grid introduces a total pressure loss between these two stations, this loss was determined experimentally and subtracted from the measured upstream total pressure. These pressure data in conjunction with inlet total temperature permit determination of test section velocity without introducing a probe into the test section. Such a probe would have been a source of extraneous vortex shedding noise.

\section{Turbulence Grid Design}

The grid employed in this study was designed based on the data of Baines and Peterson (reference 13). The grid was bi-planar consisting of bars with a dimension of $2.54 \mathrm{~cm}$ transverse to the stream and $1.91 \mathrm{~cm}$ parallel to the stream. The grid mesh was square with a mesh size (center-to-center bar spacing) of $13.3 \mathrm{~cm}$ and the solidity was 0.35 . As noted in reference 14 , turbulence downstream of square-mesh grids with this mesh-to-bar size ratio becomes practically isotropic in an axial distance of 10 to 15 mesh lengths. In the present experiment the axial separation of grid and airfoil centerline was 11.7. The grid Reynolds number based on mean velocity (through the mesh) and bar size was approximately 50,000 for the lowest tunnel speed employed in this study $(40 \mathrm{~m} / \mathrm{sec})$. 
Turbulence generated by a grid has been found to be anisotropic with the axial component of higher intensity than the transverse components. Since stream contraction causes relative amplification of the transverse components (references 15 and 16), isotropy can be improved by providing a contraction between the grid and test section. Reference 17 recommends a contraction with an area ratio of about 1.3. In the present experiment this ratio was 2.2. As discussed subsequently, test section turbulence at the airfoil location was found to be nearly isotropic with an intensity on the order of 4 to 5 percent.

\section{Test Program}

Far-field noise and surface pressure spectra were measured at tunnel velocities of $40,60,90,120$ and $165 \mathrm{~m} / \mathrm{sec}$ for airfoil geometric angles of attack of both 0 and $8 \mathrm{deg}$. These velocities correspond to free stream Mach numbers of approximately $0.12,0.18,0.27,0.36$ and 0.50 . Cross-correlations were performed between surface mounted microphones to define surface pressure cross-PSD and correlation lengths as a function of frequency. Cross-correlations were performed between surface mounted microphones and far-field microphones to assist in determining the chordwise distribution of noise sources. Cross-correlations were also performed between hot wire turbulence signals and surface and far-field microphones. Spectral and correlation characteristics of the horizontal and vertical components of turbulence incident on the airfoil were also determined. 
DEFTNITION OF THE INCIDENT TURBULENCE FTELD

\section{Required Measurements}

Acoustic radiation by an airfoil at an angle of attack of zero in the presence of inflow turbulence is associated with unsteady lift caused by the transverse turbulence component that is normal to both span and chord (in this experiment, the vertical component). At angle of attack, the axial component also affects lift. For the zero angle of attack case, noise generation is dependent upon the distribution of intensity and axial length scale across the airfoil span as well as the extent to which the vertical velocity component is correlated in the spanwise direction. Measurements were therefore required to establish the extent of spanwise uniformity (lateral. homogeniety), spectra, axial length scale and the cross-PSD of the vertical velocity component in the spanwise direction. While prediction of overall sound pressure level would require only the overall spanwise cross-correlation of the vertical component, prediction of spectra requires knowledge of the spanwise cross-correlation as a function of frequency (i.e., spanwise cross-PSD). Measurement of axial component properties was also required to assess the turbulence model employed in the analysis and to consider angle of attack effects.

The turbulence measurements discussed below were obtained with the airfoil removed and, for convenience, at an axial location corresponding to the airfoil mid-chord. Previous measurements (reference 18) obtained with the grid employed in this study demonstrated that turbulence properties have negligible variation over an axial distance of one airfoil half-chord $(11.4 \mathrm{~cm})$, hence, the measurements can be considered to apply to the airfoil leading edge position.

\section{Spanwise Homogeniety}

The turbulence field sufficiently far downstream of grids such as those employed in this study is approximately homogeneous in planes normal to the tunnel axis; that is, statistical properties of the turbulence do not depend on absolute position in the two transverse directions (vertical and lateral). Figure 3 displays measurements of the axial and vertical turbulence component intensities for various velocities as a function of test section spanwise distance that were taken to assess the degree of turbulence homogeniety in the spanwise direction. The spanwise distribution is shown to be relatively uniform with the maximum deviation from the span average being in the range from 5 to 9 percent of the span average intensity for all eight tranverses. 
The axial and vertical component span average intensities were approximately the same, differing in absolute percentage by 0.14 to 0.44 percent. As a percentage of the intensities this represents a range from about 3 to 10 percent. For the purpose of noise prediction the turbulence was assumed to be homogeneous in the spanwise direction with intensities given by the figure 3 span average values.

\section{Turbulence Iength Scales}

Shown in figure 4 are autocorrelations of the axial and vertical components of turbulence as a function of delay time for various test velocities. The autocorrelations have been normalized by the zero delay time values. The functions are observed to decrease with delay time at a faster rate for increased test velocity as would be expected. These functions represent Eulerian correlations of the turbulence components with respect to time, $R(\tau)$. The Eulerian integral time scale of the turbulence, $J$, is given by

$$
J=\int_{0}^{\infty} R(\tau) d \tau
$$

Although figure 4 only displays $R(\tau)$ values at $100 \mu \mathrm{sec}$ increments, $J$ was calculated by graphical integration of the actual correlator output curves. These values of $\mathrm{J}$ are shown in the figure. By invoking Taylor's frozenflow hypothesis (reference 14) it is possible to calculate the longitudinal, $\Lambda_{f}$, and lateral, $\Lambda_{g}$, space integral scales from the mean velocity, $U$, and Eulerian time scales

$$
\begin{aligned}
& \Lambda_{f}=U J_{u x} \\
& \Lambda_{g}=U J_{w x}
\end{aligned}
$$

As shown in figure 4, the space integral scales are relatively independent of test velocity as would be anticipated. The ratio of lateral to longitudinal scales measured in this manner is closer to 0.8 than the value of 0.5 expected for isotropic, homogeneous turbulence.

In figure 5, these autocorrelation data are plotted in the form of spatial longitudinal, $f(x)$, and lateral, $g(x)$, velocity correlation coefficients. Taylor's hypothesis has been applied to convert from a temporal to spatial frame. While the axial component shows good agreement with the von Kármán correlation, the vertical component deviates at large axial distance. 


\section{Turbulence Spectra}

Figure 6 compares measured wavenumber spectra of the axial and vertical turbulence components at various test velocities to the empirical isotropic turbulence spectrum of Liepmann and the spectrum derived from the von Kármán interpolation formula (reference 14). In this figure, $k_{x}$ is axial wavenumber related to frequency, $f$, by $k_{x}=2 \pi f / U$ and $k_{e}$ is the wavenumber range of energy-containing eddies which is related to the axial (longitudinal) integral scale $\Lambda_{\mathrm{f}}$ by $k_{e}=3 / 4 \Lambda_{\mathrm{f}}$. The data show better agreement with the von Kármán spectrum including the $-5 / 3$ power decrease at high frequency than the Iiepmann spectrum. This would be expected due to the large Reynolds number associated with the turbulence generating grid flow. Similar behavior at large Reynolds number is shown in figure 3-13 of reference 14. As in the case of the autocorrelations discussed above, the axial component is observed to agree more closely with the von Kármán spectrum than the vertical component.

Spanwise Cross-Correlations

In figure 7, correlation coefficients of the axial and vertical components obtained by cross-correlation of two hot wire probes separated in span are shown. For the axial component, the ratio of lateral to longitudinal scale is approximately one-half as would be expected for isotropic turbulence while the vertical component ratio is less than one-half.

\section{Spanwise Cross-Spectra}

Shown in figure 8 are measurements of the spanwise cross-spectrum of the vertical turbulence velocity component for various test velocities and frequencies. This function describes the degree of spanwise correlation of the vertical velocity component as a function of frequency and is the primary input to the far-field noise spectrum prediction and surface pressure crosspower spectral density prediction method employed in this study. Shown in figure 8 as solid lines is the expression for the normalized spanwise crossspectrum that can be derived from the von Kármán interpolation formula for isotropic turbulence.

$$
\tilde{R}_{w w}\left(k_{x}, y\right)=\frac{2^{1 / 6}}{\Gamma\left(\frac{5}{6}\right)}\left(y^{*}\right)^{5 / 6}\left[K_{5 / 6}\left(y^{*}\right)-\frac{3 y^{*}}{3+8 \hat{k}_{x}^{2}} K_{1 / 6}\left(y^{*}\right)\right]
$$


In this expression, derived in Appendix $\mathrm{D}$, the $\mathrm{K}^{\prime} \mathrm{s}$ denote modified Bessel functions of the second kind and fractional order, $\hat{k}_{\mathrm{x}}$ is the ratio of axial wavenumber, $\mathrm{k}_{\mathrm{X}}$, to the wavenumber range of energy-containing eddies, $\mathrm{k}_{e}$, and $\mathrm{y}^{*}$ is normalized spanwise separation distance.

The data are well represented by the von Kármán expression provided that the normalized separation is replaced by $\hat{y}^{*}=\frac{4}{3} \mathrm{y}^{*}$. This means that the spanwise separation, $y^{*}$, is made nondimensional by an axial length scale that is only three-quarters of the value used to nondimensionalize the frequency. This relative contraction of the spanwise length scale was found necessary in previous experiments reported in reference 19. The need for such a contraction and consequent indication of slight anisotropy is of no practical importance since as noted in reference 19, the isotropy of the turbulence is irrelevant provided the required turbulence statistics can be suitably described. Based on these results it was concluded that the von Kármán expression employing $\hat{y}^{*}$ as the nondimensional separation constituted the appropriate spanwise cross-spectrum for use in the analytical prediction of surface pressures and far-field noise.

\section{Summary of Flow Measurement Results}

The incident turbulence was found to be approximately homogeneous in the spanwise direction and somewhat anisotropic. Good agreement was obtained with the von Kármán powex spectrum and also with the corresponding spanwise crossspectrum if the spanwise distance was contracted by a factor of 1.3. Based on these results, the von Kármán formulation (with contraction) was employed in the derivation of the equations for noise and surface pressure prediction. Although the noise and surface pressure prediction method is general with regard to the nature of the incident turbulence (isotropic, homogeneous turbulence is not required) and can be applied if sufficient experimental documentation of the turbulence is obtained, the applicability of the von Kármán expressions in this case eliminated inaccuracies associated with curve fitting of experimental turbulence data and simplified calculations. 
FAR FIELD ANALYSES AND RESULTS

Far Field Noise Theory

Theoretical Approach. - The present approach is based on the theoretical development of reference 20. The turbulence is assumed to be frozen and represented in terms of its spectral wavenumber components, $k_{X}$ and $\mathrm{k}_{\mathrm{y}}$. The airfoil is assumed to be a flat plate of zero thickness, and linearized theory is assumed so that the wavenumber associated with the $\mathrm{z}$ coordinate normal to the airfoil (at zero angle of attack) does not enter. End effects are ignored in calculating the airfoil response (i.e., the airfoil surface pressure is calculated as if the airfoil were infinite).

Effects of compressibility in the airfoil response function and noncompactness effects in calculation of the far-field sound are included. Compressibility effects are shown to be significant at high frequency, and their inclusion represents one of the significant improvements relative to previous theories. Finally, the assumption of large span allows simplification of the expression for far-field sound. The result for the overall lift under this approximation agrees with an expression given previously by other authors (e.g., references 19 and 21), being designated in those papers as "strip theory". However, it was not clearly pointed out in these references that the result is mathematically rigorous in the large span limit and does not require the "strip theory" approximation. A similar approximation was also made by Goldstein (reference 22). Further discussion is given in the following section relative to the meaning of the term "large span", but in general, it is a limit well suited to the problem of incident turbulence noise generation.

Theoretical Formulation. - An airfoil of chord $2 b$ and span $2 d$ is placed in a turbulent fluid with a mean flow $U$ in the axial (chordwise) direction, $x$ as shown in the sketch below. The $y$ coordinate extends in the lateral (spanwise) direction, the $z$ axis is vertical (normal to the airfoil), the origin of the coordinate system is at the center of the airfoil and the observer is in the far-field. With this notation, reference 20 shows that use of established theory and the assumption that infinite span airfoil response functions can be applied to the finite span airfoil problem, results in the following expression for the far-field sound power spectral density (PSD)

$$
S_{p p}(r, w)=\left(\frac{\omega Z P_{0} b}{c_{0} \sigma^{2}}\right) \pi u d \int_{-\infty}^{\infty}\left|\mathcal{L}\left(I_{1} k_{x}, k_{y}\right)\right|^{2} \Phi_{w w}\left(k_{x}, k_{y}\right)\left[\frac{\sin ^{2}\left(\left(k_{y}+k_{y}\right) d\right)}{\pi d\left(k_{y}+k_{y}\right)^{2}}\right] d k_{y}
$$




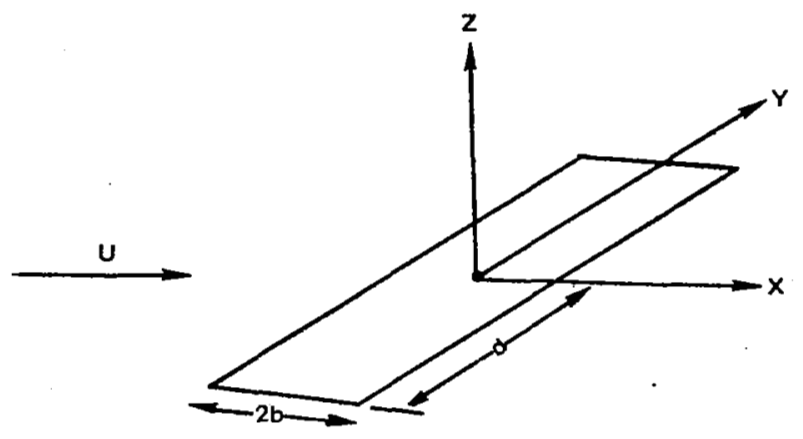

OBȘERVER where $\Phi_{\text {wW }}\left(\mathrm{k}_{\mathrm{x}}, \mathrm{k}_{\mathrm{y}}\right)$ is the two wavenumber spectrum and the effective lift,
$\mathcal{L}$, is given by:

$$
\mathcal{L}\left(\boldsymbol{I}, K_{x}, k_{y}\right)=\int_{-1}^{1} g\left(\xi, K_{x}, k_{y}\right) e^{-i \mu \xi(m-x /)} d \xi
$$

The normalized pressure jump across the airfoil, g, is defined as

$$
\Delta P\left(x_{i}, y, f_{i} k_{x}, k_{y}\right)=2 \pi P_{0} U w_{0} g\left(x, k_{x}, k_{y}\right) e^{i\left(k_{x} u t-k_{y} y\right)}
$$

where $\Delta \mathrm{P}$ is the pressure jump produced by a normal gust velocity

$$
w_{g}=w_{0} e^{i\left(k_{x}(U t-x)-k_{y} y\right)}
$$

Also, the following quantities are defined:

$$
\begin{aligned}
& K_{x}=\omega / U, K_{y}=\omega y / C_{0} \sigma \\
& \sigma^{2}=x^{2}+\beta^{2}\left(y^{2}+z^{2}\right), \mu=M K_{x} b / B^{2}, \beta^{2}=1-M^{2}
\end{aligned}
$$

In these expressions $\mathrm{k}_{\mathrm{x}}$ and $\mathrm{k}_{\mathrm{y}}$ are the axial (chordwise) and lateral (spanwise) wavenumbers of the turbulence, respectively. Additional quantities are defined in the List of Symbols. It should be noted that equations $(6 a-6 c)$ are the complex conjugates of the corresponding equations given in reference 20. This results in a positive value $K_{X}=\omega / U$ rather than the negative value $K_{X}=-\omega / U$ of reference 20 when the time Fourier transform is defined as

$$
F(\omega)=\frac{1}{2 \pi} \int_{-\infty}^{\infty} f(t) e^{-i \omega t} d t
$$


The function $\left(\sin ^{2} \xi \mathrm{d}\right) /\left(\xi^{2} \pi \mathrm{d}\right)$ in equation (4) behaves like a delta function of $\xi$ for large d. As was shown in reference 20 , when the acoustic wavelength, $\lambda$, is much smaller than the airfoil semispan, $d$, equation (4) can be simplified to

$$
S_{p p}(r, \omega)=\left(\frac{\omega z P_{0} b}{C_{0} \sigma^{2}}\right)^{2} \pi \cup d\left|\mathcal{L}\left(\underline{r}, K_{x}, K_{y}\right)\right|^{2} \Phi_{w w}\left(K_{x}, K_{y}\right)
$$

Under this limitation (which can be written $\mathrm{MK}_{\mathrm{X}} \mathrm{d} \gg \mathrm{I}$ ) the airfoil loading becomes concentrated within the order of a wavelength from the airfoil leading edge and finite span effects are limited to a distance on the order of a wavelength from the ends. Thus, under the limit $\lambda \ll d$, it is not necessary to assume large airfoil aspect ratio for equation (7) to hold. When the aspect ratio, $A R$, is large, the restriction $M_{X} d \gg I$ can be relaxed to $\mathrm{K}_{\mathrm{x}} \mathrm{d} \gg 1$. Under this restriction, end effects are limited to within a chordlength from the ends and can again be neglected. Equation (7) is thus exact for both the limits

and

$$
\operatorname{MK}_{\mathrm{x}} d \rightarrow \infty
$$

$$
K_{x} d-\infty \text { with } A R \rightarrow \infty
$$

The airfoil response functions used to calculated $\mathcal{L}$ will be approximations, one for the low frequency regime and one for the high frequency regime. Together they give good approximations to the airfoil response for all frequency, as discussed in reference 23.

For the low frequency regime, $\mu<0.4$, the solution of Amiet (reference $24)$ is used. This small $\mu$ solution is correct to $O(\mu)$ and neglects terms $O\left(\mu^{2}\right)$ and higher. It is similar to the solution of osborne (reference 25) which had neglected a term of $O(\mu)$. Further discussion of the solution is given in references 23,26 and 27 . The solution for the pressure distribution produced by a gust given by equation ( $6 \mathrm{~b})$ with $k_{\mathrm{y}}=0$ on an airfoil situated between $-1 \leq \bar{x} \leq 1$ is shown in reference 24 to be

$$
g\left(x, k_{x}, 0\right)=\frac{1}{\pi \beta} \sqrt{\frac{1-\bar{x}}{1+\bar{x}}} S\left(\bar{k}_{x}^{*}\right) e^{i \bar{x}_{x}^{*}\left(m^{2} \bar{x}+f(M)\right)}, \mu<0.4
$$

where

$$
f(M)=(1-\beta) \ln M+\beta \ln (1+\beta)-\ln 2
$$


and $S$ is the classical Sears function (reference 1 ). The overbar indicates a variable nondimensionalized by the semichord $b$. The result for non-zero $\mathrm{k}_{\mathrm{y}}$, needed when the observer is not in the $\mathrm{y}=0$ plane, can be obtained using the similarity rules of Graham (reference 28). Introducing equation

(9) into equation (5) gives

$$
\mathcal{L}\left(I, k_{x}, 0\right)=\frac{1}{B} S\left(\bar{k}_{x}^{*}\right)\left[J_{0}(\mu x / \sigma)-i J_{1}(\mu x / \sigma)\right] e^{i k_{x}^{*}(m)}, \mu<0.4
$$

Since only the absolute value is needed, this can be simplified. Equation (10) already ignores terms of $O\left(\mu^{2}\right)$. To be consistent, the absolute value of $J_{0}-i J_{I}$ can be approximated by 1 , giving

$$
|\mathcal{L}|=\frac{1}{\beta}\left|s\left(\bar{k}_{x}^{*}\right)\right|
$$

A simple, but accurate, approximation to the sears function which was used in the calculations is

$$
s\left(\bar{k}_{x}\right)=\left(\frac{1}{1+2.4 \bar{k}_{x}}+2 \pi \bar{k}_{x}\right)-1 / 2
$$

Although this approximation loses the logarithmic behavior of the Sears function for small $k_{x}$, the accuracy is quite good.

For high frequency the solution used is that of Adamczyk which was derived by an iteration procedure similar to that of references 29-30. It consists of a series of corrections alternately to the leading and trailing edges. The first two terms given by Adamczyk (reference 3l) will be used here. An alternate derivation and further comparison with numerical results is given in references 23 and 32. The first term alone can be used for $\mu>$ 0.75 . Here the first two corrections will be used which extends the range to $\mu>0.4$; this is the changeover between the high and low frequency two-dimensional solutions used in the present calculations. The first two terms of the Adamczyk solution for the pressure jump, $g$, of an airfoil situated between $-1 \leq x \leq l$ are given in references 23,31 and 32 as (presented here are the $k_{\mathrm{y}}=0$ Iimits and the complex conjugate of the solutions given in reference 31. Also, both references 23 and 31 use different definitions of $\mathrm{E}$ ).

$$
g_{1}\left(x, k_{x}, 0\right)=\frac{1}{\pi \sqrt{\pi(1+M) \bar{k}_{x}(1+\bar{x})}} e^{-i\left[\mu(1-M)(1+\bar{x})+\pi / 4-\bar{k}_{x}\right]}
$$

$$
g_{2}\left(x, k_{x}, 0\right) \approx \frac{1}{\pi \sqrt{2 \pi(1+M) k_{x}}}\left[(1+i) E^{*}(2 \mu(1-\bar{x}))-1\right] \mathrm{e}^{-i\left[\mu(1-M)(1+\bar{x})+\pi / 4-\bar{k}_{x}\right]}
$$


where

$$
E^{*}(x)=\frac{1}{\sqrt{2 \pi}} \int_{0}^{x} e^{-i \xi} \frac{d \xi}{\sqrt{\xi}}
$$

is a combination of Fresnel integrals.

Introducing equations ( $13 a$ and $b$ ) into equation (5) gives

$$
\begin{gathered}
\mathcal{L}_{1}\left(r, k_{x}, 0\right)=\frac{1}{\pi} \sqrt{\frac{2}{(1+M) k_{x} \Theta_{1}}} E^{*}\left(2 \oplus_{1}\right) e^{i \oplus 2} \\
\mathcal{L}_{2}\left(I, k_{x}, 0\right) \approx \frac{1}{\pi \oplus_{1} \sqrt{2 \pi(1+M) \bar{k}_{x}}} e^{i \oplus_{2}}\left\{i \left(1-e^{\left.-i 2 \Theta_{1}\right)}\right.\right. \\
+(1-i)\left[E^{*}(4 \mu)-\sqrt{\frac{2}{1+x / \sigma}} e^{\left.\left.-i 2 \oplus_{1} E^{*}\left(2 \mu\left(1+\frac{x}{\sigma}\right)\right)\right]\right\}}\right. \\
\ell=L_{1}+\mathcal{L}_{2}
\end{gathered}
$$

where $\mathbb{Q}_{1} \equiv \mu(1-x / \sigma)$ and $\mathbb{B}_{2} \equiv \bar{k}_{x}^{*}(1-M x / \sigma)-\pi / 4$

For use in equation (7), equations ( $15 \mathrm{a}$ and $15 \mathrm{~b}$ ) are first added, and then the absolute value is taken whereupon the function $\Theta_{2}$ drops out.

Equations (6), (11), (12), (14) and (15) in conjunction with the two wavenumber spectrum of the vertical velocity component of the turbulence, $\Phi_{W W}\left(k_{x}, k_{y}\right)$ (given as equation (D3) of Appendix D) are those used in the present study to predict far-field noise in the $y=0$ plane. In comparing with experimental data the physically realizable one-sided PSD, defined for positive frequencies only, $G_{p p}=2 S_{p p}$, is employed and a factor of $2 \pi$ is accounted for in expressing results in terms of unit frequency rather than unit circular frequency.

Shear Layer Refraction Effects. - To compare with data, a correction must be applied to account for refraction by the tunnel shear layer. For this purpose the open jet wind tunnel refraction corrections of Amiet (reference 33) were used. This necessitates both a directivity angle and an amplitude correction. Rather than correct the data, however, the data were left "as measured" and the corrections were applied to the theory in order to find predicted sound levels in the presence of the shear layer. The corrections given in reference 33 assume a plane, zero-thickness shear layer. The shear 
layer correction is a function of observer angle, Mach number and the ratio of the normal distance between source and shear layer and the sourceobserver distance. The correction, however, is independent of frequency. Plots of the corrections which were applied to the theoretical results are shown in figure 9. Applying the corrections in this manner transforms the predictions to correspond to sound measured in the presence of the tunnel shear layer. Thus, the corrections were used in the inverse manner relative to their normal use, i.e., to transform measured data to values which would be measured with a free stream extending to infinity. This allowed the experimental data to be presented "as measured" with no shear layer correction.

Airfoil Response Function Compressibility Effects. - The importance of using airfoil response functions that include the effect of compressibility is shown in figure 10. This figure shows the results of a calculation using the airfoil response functions that include compressibility compared with the same calculations using the incompressible airfoil response function (the classical sears function) times the Prandtl-Glauert factor, l/B. The results agree closely at low frequency as would be expected, but at high frequency the difference can be significant. This is because the sears function behaves as $k_{\mathrm{x}}-1 / 2$ for large $k_{\mathrm{x}}$ while the compressible response function behaves as $k_{\mathrm{x}}^{-1}$

Sound Directivity Considerations. - It is of interest to examine the expression for the far-field sound in the limits of low and high frequency (small and large $\mu$ ). The retarded coordinates, denoted by the subscript, e, are useful in expressing these results; $r_{\mathrm{e}}$ is the retarded distance and $\theta_{e}$ the retarded angle measured relative to the positive $x$ axis (downstream direction). Retarded coordinates are discussed more fully in Appendix $\mathrm{E}$ and reference 20 . The relations between the retarded coordinates and the real time coordinates are

$$
\begin{gathered}
\cos \theta_{e}=\sqrt{1-M^{2} \sin ^{2} \theta} \cos \theta-M \sin ^{2} \theta \\
r e \sin \theta_{e}=r \sin \theta
\end{gathered}
$$

In the low frequency limit the airfoil response $|\mathfrak{L}|$ given by equation (11) reduces to $1 / \beta$. For an observer in the $y=0$ plane, equation ( 7 ) then becomes

$$
\operatorname{Spp}(r, \omega)=\left(\frac{\omega_{b} \rho_{0}}{\beta c_{0} r_{e}}\right)^{2} \pi u d \frac{\sin ^{2} \theta_{e}}{\left(1+M \cos \theta_{e}\right)^{4}} \Phi_{w w}\left(K_{x}, 0\right)
$$

The directivity pattern, $\sin ^{2} \theta_{e} /\left(1+M \cos \theta_{e}\right)^{4}$, for this case is exactly that which would be obtained for a compact dipole moving at Mach number $\mathrm{M}$. 
For the high frequency limit the function $\mathcal{L}_{1}$ given by equation (15a) is much greater than $\mathfrak{L}_{2}$ (except for an observer near the positive $\mathrm{x}$ axis) so that $\mathcal{S}_{2}$ can be ignored. If $\mathcal{L}_{1}$ (for an observer in the $y=0$ plane) is expanded for large $\mu$, equation (7) becomes

$$
S_{P P}(\underline{r}, \omega)=\frac{2 P_{0}^{2} U d M}{\pi r_{e}^{2}} \frac{\cos ^{2}\left(\theta_{e} / 2\right)}{\left(1+M \cos \theta_{e}\right)^{3}} \Phi_{w w}\left(K_{x}, 0\right)
$$

These two expressions are plotted in figure 11. The curves have been normalized to 1 for the 90 degree angle. It will be noted that there is a significant difference between the two curves, showing the importance of accounting for noncompactness effects at high frequency. (Because of the approximation used in deriving equation (17), the high frequency directivity in figure 11 is that for an airfoil with a semi-infinite chord. Thus, a finite sound level is predicted for $\theta \rightarrow 0$ whereas a finite chord airfoil would be predicted to have zero sound there. This affects the curve only over a small angle around $\theta=0$.)

The velocity dependence of equations ( $17 \mathrm{a}$ and $\mathrm{b}$ ) is also of interest. If the percent turbulence level and the axial wavenumber $K_{X}(=\omega / U)$ are kept fixed as $U$ is varied, and $M$ is small, the acoustic energy in a fixed percent frequency bandwidth behaves as $U^{6}$ at low frequency and $U^{5}$ at high frequency, as noted from equations ( $17 \mathrm{a}$ and $\mathrm{b}$ ). (Note that since $\mathrm{K}_{\mathrm{X}}$ is kept fixed, w must vary as U.) These results are similar to those found elsewhere (see e.g., references 4 and 34). At non-negligible Mach number other factors in equation (17), such as $\left(1+M \cos \theta_{e}\right)$, become important and alter the velocity dependence from the simple fifth and sixth powers. The parameter $\mu$, critical in deciding whether the low or high frequency limits are applicable, is proportional to chord, $c$, divided by acoustic wavelength, $\lambda$. Thus, when $\lambda \gg c$ the low frequency limit can be used, while if $\lambda \ll c$ the high frequency limit is applicable.

Far Field Experimental Results

Typical Results. - Far-field noise spectra were obtained at free stream velocities of $40,60,90,120$ and $165 \mathrm{~m} / \mathrm{sec}$ at angles relative to the upstream direction of $70,90,105,120,130$ and $140 \mathrm{deg}$ for both zero and $8 \mathrm{deg}$ geometric angle of attack. Shown in figure 12 are the results for $90 \mathrm{deg}$. The ordinate is "spectrum level" obtained as discussed in the section entitled "Instrumentation." The data signals were filtered prior to analysis by a $150 \mathrm{~Hz}$ high pass filter. Data are presented for an empty test section and for airfoil angles of attack of zero and $8 \mathrm{deg}$. The empty test section data 
represent noise from other sources such as the tunnel free-jet collector and shear layer and constitute the background noise for the experiment. In figure 12, background noise has not been subtracted from measured airfoil noise. The turbulence induced airfoil noise is observed to be broadband and of high intensity, standing out as much as $15 \mathrm{~dB}$ above background at the low frequencies where the noise is most intense. Increase of airfoil angle of attack from zero to $8 \mathrm{deg}$ is seen to cause a small but measurable increase in noise over the mid-to-high frequency range.

Comparison of Theoretical and Experimental Spectral Results. - Shown in figure 13 is a comparison between theory and experiment for the noise spectra directly above the airfoil at an airfoil angle of attack of zero. The directivity angle, $\phi_{\mathbb{M}}$, is the angle relative to the upstream direction and is therefore $90 \mathrm{deg}$ for this case $(\phi=180-\theta)$. The data represent measurement at $90 \mathrm{deg}$ in the presence of the tunnel shear layer. The theoretical predictions shown by solid lines include the shear layer refraction correction discussed in the previous section entitled "Shear Layer Refraction Effects" and can therefore be compared directly to measured data acquired in the presence of the tunnel shear layer.

The experimental data points have been obtained by subtracting measured tunnel background noise from the measured spectra. Flagged symbols denote data points for which the difference between airfoil and background noise was between $4.3 \mathrm{~dB}$ and $2.2 \mathrm{~dB}$ thus requiring corrections of 2 to $4 \mathrm{~dB}$ in measured airfoil levels. These data are subject to greater uncertainty than the unflagged points for which corrections of less than $2 \mathrm{~dB}$ were required. Data requiring corrections greater than $4 \mathrm{~dB}$ have not been plotted since an uncertainty greater than one or two decibels in absolute level could exist.

The agreement between theory and experiment is considered good, particularly for the low-frequency noise that dominates the spectra and for the high Mach numbers most relevant to helicopter rotor, propeller and turbomachinery noise. The average absolute difference between the theoretical curves and data shown in figure 13 (excluding flagged symbols) is $0.5 \mathrm{~dB}$ for $165 \mathrm{~m} / \mathrm{sec}, 1.5 \mathrm{~dB}$ at $120 \mathrm{~m} / \mathrm{sec}, 3 \mathrm{~dB}$ at $90 \mathrm{~m} / \mathrm{sec} .1 .5 \mathrm{~dB}$ at $60 \mathrm{~m} / \mathrm{sec}$ and $4 \mathrm{~dB}$ at $40 \mathrm{~m} / \mathrm{sec}$. Significant deviations between theory and experiment are generally observed at high frequency with the disagreement greatest at low velocity. Such behavior would be expected due to finite airfoil thickness effects not accounted for by the theory. When the gust wavelength decreases to a length comparable to the airfoil thickness in the vicinity of the leading edge, significant errors would be anticipated. Since gust wavelength 
is given by the ratio of mean velocity to frequency, an order of magnitude criterion for breakdown of theory can be expressed as $U / f^{t} t_{A} \leq l$ where $f$ is sound frequency, $U$ the velocity and, $t_{A}$, the airfoil thiciness. The observed disagreement is approximately $5 \mathrm{~dB}$ when the above equality is satisfied. Although a rigorous explanation for this breakdown cannot be given, an eddy small in comparison with a leading edge thickness dimension would not be expected to produce the airfoil lift fluctuation that would obtain if the airfoil had zero thickness and consequently appeared to the eddy as a knife edge. An implication of this result is that noise reductions greater than those predicted by theory may be achieved by reducing the ratio of turbulence scale to thickness. Experimental confirmation of this anticipated result would be of interest. Improvement of noise prediction theory by extending airfoil lift theories to include finite thickness would also be useful.

Comparison of Theoretical and Experimental Directivity Results. - Shown in figure 14 is a comparison between theory and experiment for the far-field airfoil noise directivity as a function of frequency at an airfoil angle of attack of zero. All data that were at least $2.2 \mathrm{~dB}$ above tunnel background noise have been plotted. As in the case of the 90 deg spectral comparisons above, the theoretical predictions include a refraction correction to permit direct comparison with measurements obtained outside the wind tunnel shear layer. The directivity angle, $\phi_{\mathrm{M}}$, is measured relative to the upstream axis (i.e., $\phi_{\mathrm{M}}=180-\theta$ ). The levels have been corrected to account for background noise and the symbol flags have the same meaning as above. The agreement is observed to be good except for low values of U/ft $\mathrm{A}_{\mathrm{A}}$, as in the case of the $90 \mathrm{deg}$ spectral comparison. Of interest is the theoretical prediction of a progression from a smooth directivity pattern nearly symmetrical with $90 \mathrm{deg}$ at low frequency to an aft quadrant dominated, wavy pattern at high frequency. This is partially confirmed by the data.

Both of the above effects are attributed to source noncompactness at high frequency. At low frequency (large gust wavelength) the airfoil chordwise pressure distribution is in phase. This distribution can be modeled in the far-field as a point dipole which has a symmetrical directivity pattern about $90 \mathrm{deg}$ (sin behavior). With increased frequency, the airfoil leading edge dominates and the pattern tends toward the $\sin \phi / 2$ behavior associated with edge radiation from a semi-infinite plate. Waviness in both frequency spectra and directivity patterns at high frequency are believed to be associated with the cancellation of waves from negative and positive portions of the airfoil chordwise pressure distribution. 
In addition to supporting the airfoil turbulence noise theory employed here, the data provide indirect substantiation for the wind tunnel shear layer refraction correction applied to the theoretical directivity predictions. As shown in figure 14, the agreement at angles other than 90 deg is similar to that obtained at $90 \mathrm{deg}$ where refraction effects are small. There exist other experimental data which tend to support the refraction correction applied here in a more direct manner (reference 33).

A dipole radiation pattern is often assumed in predicting directivity patterns associated with incident turbulence noise. The good agreement between prediction and experiment shown in figure 14 in conjunction with the significant deviation between the classic dipole pattern and the predictions of theory at high frequency (figure 11) demonstrate the limitations of this dipole pattern assumption. At the higher frequencies of most importance to perceived noise, an edge type radiation pattern may be more relevant in the prediction of turbulence induced noise associated with full-scale helicopter rotors, propellers, and turbofan engines.

\section{Importance of Incident Turbulence as a Noise Mechanism}

Based on the results of the present study and previous noise data obtained in the UTRC Acoustic Tunnel with the same airfoil, isolated airfoil noise mechanisms can be ranked in terms of their relative importance. For a twodimensional isolated airfoil, the operative noise mechanisms are (1) incident turbulence, (2) the turbulent boundary layer, (3) stalled airfoil flow and (4) discrete frequency vortex shedding.

Incident turbulence of approximately 4 percent intensity and 15 percent chord length scale was observed in the present study to cause far-field noise with an intensity as much as $15 \mathrm{~dB}$ above tunnel background noise. The sum of direct radiation from an attached turbulent boundary layer and its interaction with the airfoil trailing edge (attached flow trailing edge noise) obtained under low turbulence inflow conditions, however, was found (reference 10) to be undetectable above tunnel background noise. The turbulent boundary layer is therefore a much weaker noise source.

In stalled airfoil flow studies (reference 11), an increase of several decibels above tunnel background noise was found. The operative noise mechanism was demonstrated to be interaction of stall-generated eddies with the airfoil trailing edge. This mechanism is therefore much stronger than boundary layer noise but an order of magnitude weaker than the incident turbulence considered here. The final isolated airfoil mechanism is vortex 
shedding noise associated with a trailing edge laminar boundary layer (reference 10). While comparable in intensity to the present incident turbulence noise, the mechanism disappears when both suction and pressure surface boundary layers are turbulent at the trailing edge. Since this situation obtains in almost all full-scale applications, this mechanism is not of practical importance.

Based on these consideration, incident turbulence is concluded to be an important isolated airfoil noise mechanism. For the inflow turbulence properties considered here, it strongly dominates the other practically important mechanisms. In full-scale applications, its relative importance would depend upon the intensity and scale of the incident turbulence flow field. The theory discussed above can be used to estimate the turbulence induced noise in such situations.

\section{Approximate Expressions for Far-Field Noise}

While the complete solutions for far-field noise given in this section are relatively easy to program and must be used where high accuracy or general directivity angle predictions are required, Appendix E provides approximate expressions for the noise at $\phi_{\mathrm{m}}=90$ deg suitable for estimation purposes. Predicted levels are given in third-octave bandwidth. Appendix E also discusses application of these expressions to rotating blade noise prediction and acoustic wind tunnel measurements.

Far Field Summary

For the low frequencies that dominate the airfoil noise spectra and high Mach numbers most relevant to practical applications, good agreement in both spectra and directivity (typically on the order of 1 or $2 \mathrm{~dB}$ ) was observed between experiment and a theory capable of absolute level prediction without empirical constants. Significant disagreement was noted where finite airfoil thickness effects not accounted for by theory would be expected to become important. The good agreement provided indirect support for the shear layer refraction corrections applied to the data. 
SURFACE PRESSURE ANTALYSES AND RESULTS

Surface Pressure Theory

The airfoil surface pressure for any frequency can be found by summing the airfoil response to all the spectral gust components contributing to that frequency. As given by equation(11) of reference 20, the cross-PSD of the surface pressure $\mathrm{S}_{\mathrm{qq}}$ is

$$
S_{q q}\left(x_{1}, x_{2}, \eta, \omega\right)=2 U\left(\pi \rho_{0}\right)^{2} \int_{0}^{\infty} g^{*}\left(x_{1}, k_{x}, k_{y}\right) g\left(x_{2}, k_{x}, k_{y}\right) \Phi_{w w}\left(k_{x}, k_{y}\right) \cos \left(k_{y} \eta\right) d k_{y}
$$

(Note that in reference $20, S_{Q Q}$ referred to pressure jump across the airfoil, not surface pressure, hence an additional factor of $1 / 4$ was introduced here.)

For the calculation of far-field noise it was possible to simplify the $k$ integral in equation (4). This is due to the fact that an integral over the span was taken, resulting in a cancelling effect for all $\mathrm{k}$ spectral components except the one giving the entire noise contribution at that particular observer position. In calculating unsteady surface pressure, the absence of an integral over span means that the $k$ integral cannot be simplified. Thus, the integration in equation(18) was carried out numerically to obtain the predictions of surface pressure cross-PSD.

For large spanwise separation, $\eta$, the integrand in equation (18) oscillates rapidly, and is thus difficult to evaluate numerically. For this reason Filon's method (e.g., reference 35) which is directed toward this type of integral, was used.

Whereas for the calculation of far-field noise only the airfoil response for the parallel gust was needed, for calculating surface pressure, results for both the parallel compressible gust and the skewed incompressible gust are needed. The approximate results used for the parallel compressible gust are the same as those used in the calculation of far-field noise. As for the parallel gust case, the calculation for the skewed incompressible gust was divided into two regimes: small $\mathrm{k}$ and large $\mathrm{k}_{\mathrm{y}}$.

The solution for small $\mathrm{k}_{\mathrm{y}}$ is that of Amiet (reference 36), which gives for the normalized pressure jump on an airfoil between $-1 \leq \bar{x} \leq 1$

$$
g\left(x, k_{x}, k_{y}\right)=\frac{1}{\pi} S\left(\bar{k}_{x}\right) \sqrt{\frac{1-\bar{x}}{1+\bar{x}}} e^{i \bar{k}_{x} f^{\prime}\left(k_{y} / k_{x}\right)} \quad \bar{k}_{y}<0.3
$$


where

$$
f^{\prime}(\xi)=\left(\sqrt{1+\xi^{2}}-1\right)(i \pi / 2-\ln \xi)+\sqrt{1+\xi^{2}} \ln \left(1+\sqrt{1+\xi^{2}}\right)-\ln 2
$$

This approximate solution was derived from the corresponding solution of Amiet (equation(9)) for a parallel compressible gust using the Graham (reference 28) similarity rules.

The solution for large $k_{y}$, as for the parallel gust case, was obtained by Adamczyk using the aforementioned iteration technique. This solution is, in fact, mothematically related to the result for the parallel compressible gust case through the similarity rules of Graham (reference 28), just as the above small $\mathrm{k}_{\mathrm{y}}$ solution is related to the small $\mu$ solution. The first two terms given by Adamczyk (reference 3I) were shown in reference 32 to be quite accurate for $\mathrm{k}_{\mathrm{y}}>0.25$ when compared with the numerical results of Graham (reference 37). For the present calculations, the two-term solution was used over the range $k_{y}>0.3$. For an airfoil situated between $-1 \leq \bar{x} \leq 1$, the solution is

$$
\begin{gathered}
g_{1}\left(x, k_{x}, k_{y}\right)=\frac{1}{\pi \sqrt{\pi\left(\bar{k}_{y}+i \bar{k}_{x}\right)(1+\bar{x})}} e^{-\bar{x} \bar{k}_{y}+\bar{k}_{x}} \\
g_{2}\left(x, k_{x}, k_{y}\right)=\frac{1}{\pi \sqrt{2 \pi\left(\bar{k}_{y}+i \bar{k}_{x}\right)}}\left[\operatorname{erf}\left(\sqrt{2 \bar{k}_{y}(1-\bar{x})}\right)-1\right] e^{-\bar{x} \bar{k}_{y}+\bar{i}_{x}}
\end{gathered}
$$

where

$$
\operatorname{erf}(x)=\frac{2}{\sqrt{\pi}} \int_{0}^{x} e^{-t^{2}} d t
$$

is the error function.

Although Adamczyk gives directly the response function for a skewed compressible gust, this was broken down into the parallel compressible gust result (equations (13)) and the skewed incompressible gust result (equations (20)) since these were the limiting cases which were checked against numerical results. Graham's similarity rules (reference 28) were then used to relate the general skewed compressible gust case to one of these two simpler results. This gives a more precise understanding of the accuracy to be expected of the general solution. The approximate solutions used herein are compared with exact numerical results in Table I showing that the approximate solutions are accurate to within a few percent of the exact solutions. 
Introducing these airfoil solutions into equation (18) along with equation (D3) for the turbulence allows calculation of the cross-spectrum of the surface pressure. Figure 15 shows the calculated cross-spectrum magnitude as a function of spanwise separation. From this figure it can be seen that the correlation length near the leading edge can be significantly smallex than that near the trailing edge. Defining the spanwise correlation length, $\ell^{\prime}$, on the airfoil surface to be the distance at which the cross-spectrum at a given frequency falls to $1 / e$ of its value for zero separation, gives the plot of spanwise correlation length as a function of chordwise position shown in figure 16(a). Near the trailing edge the correlation length, $l^{\prime}$, is slightly greater than the chord, $c$, whereas near the leading edge $l_{\mathrm{y}}^{i}$ is less than $c / 5$, thereby illustrating the significant variation in $h_{y}^{\prime}$ which ${ }^{\mathrm{y}}$ can take place over the chord. Figure 16(b) shows the predicted spectrum level for zero separation as a function of chordwise position. This is the level that was used to nondimensionalize the results shown in figure 15.

It is interesting to note that the correlation length $h_{y}^{\prime}(\omega, x)$ on the airfoil surface, defined as above, becomes comparable at the airfoil leading edge to the correlation length $\ell_{\mathrm{y}}(\omega)$ for the turbulence, found by integration of the turbulence cross-spectrum over spanwise separation. For the von Kármán spectrum, the result given in reference 20 is

$$
\ell_{y}(\omega)=\frac{8}{3}\left[\frac{\Gamma(1 / 3)}{\Gamma(5 / 6)}\right]^{2} \Lambda_{f} \frac{\hat{K}_{x}^{2}}{\left(3+8 \hat{k}_{x}{ }^{2}\right) \sqrt{1+\hat{R}_{x}^{2}}}
$$

For a turbulence length scale, $\Lambda_{\mathrm{f}}=3.0 \mathrm{~cm}$, and frequency, $f=400 \mathrm{~Hz}$, $b \mathrm{y}$ is found to be $2.5 \mathrm{~cm}$, while $h_{y}^{\prime}$ at the leading edge is shown by figure 16(a) to be $3.9 \mathrm{~cm}$. (Note the slight diffexence in the definitions of $l_{y}$ and $l_{y}^{\prime}: b_{y}$ is found by integration over spanwise separation whereas $b_{y}^{\prime}$ is the spanwise separation at which the cross-correlation falls by a factor of I/e.) The surface pressure correlation length behavior described above is in agreement with intuition. At the leading edge the turbulence correlation length is $l_{\mathrm{y}}(\omega)$. Surface pressures near the leading edge would be expected to have a similar length scale because they arise from the turbulence - leading edge interaction. Since the airfoil lift adjustment occurs by propagation of acoustic waves, the influence of an eddy would be expected to be extended over a greater spanwise extent at aft locations.

Figure 17 shows that the correlation length on the airfoil is a function of frequency. (This is true as well for the turbulence correlation length $\ell_{y}$ given by equation (22).) It is interesting to note from figure 17 that the cross-PSD for a given frequency and separation can be 180 degrees out of phase with the value for zero separation. This is shown by the several humps in the 
$2000 \mathrm{~Hz}$ curve. This effect can lead to overestimation of the far-field noise when calculated from surface measurements if the point where the curve falls to $I / e$ of its value is used to define $l_{\mathrm{y}}^{\prime}$ : i.e., the contribution to the noise produced by the first hump in the curve is partially cancelled by the second hump. This problem of oscillating correlation function behavior is discussed in reference 14 relative to turbulence theory.

The magnitude of the cross-correlation shown in figure 17 goes to zero at certain spanwise positions only because the two points at which the crosscorrelation is made are at the same chordwise position. This causes the crossspectrum to be a real function. When the points are at different chordwise positions, the cross-spectrum will have both a real and an imaginary part, both of which do not generally become zero simultaneously. In this case, rather than switching in phase from zero to 180 degrees instantaneously as in figure 17, the phase makes a gradual change. The cross-spectrum curve plotted versus spanwise separation is still oscillatory in behavior, however, with the second hump tending to cancel the first as in figure 17.

Figure 18 shows the normalized cross-spectrum $\mathrm{s}_{12} / \sqrt{\mathrm{S}_{11} \mathrm{~S}_{22}}$ with finite chordwise separation but zero spanwise separation for points 1 and 2 . As expected, the cross-PSD decreases with chordwise separation.

The above discussion shows the complicated manner in which the crossspectrum of the surface pressure can behave. These complexities are therefore inherent in a far-field noise prediction procedure dependent upon surface presure measurements. A conclusion of this report is that the surface pressure approach is less desirable than one which proceeds directly from the incident turbulence statistics. Not only is the latter easier to carry out, but it represents a complete solution to the problem. Further comments on this subject are contained in the section entitled, "Prediction of Far-Field INoise from Surface Pressure Measurements".

In the comparisons with data given in the next section, theoretical surface pressure PSD and cross-PSD are expressed in terms of one-sided frequency functions and amplitudes were calculated in terms of unit frequency. To calculate surface pressure spectra (PSD) rather than cross-PSD, $x_{1}$ is taken equal to $x_{2}$ and $\eta=0$ in equation (18).

\section{Surface Pressure Experimental Results}

Typical Spectral Results. - Surface pressure spectra were obtained at free stream velocities of $40,60,90,120$ and $165 \mathrm{~m} / \mathrm{sec}$ at positions of $15,30,38$, 50 and 70 percent chord on the airfoil upper surface for both zero and 8 deg angle of attack. Shown in figure 19 are typical spectra for zero angle of 
attack as a function of velocity. At the two highest velocities, 15 percent chord levels were significantly higher than those at aft positions for aII frequency while at the lower velocities aft microphone levels exceeded 15 percent chord levels at high frequency. This latter behavior is believed due to phenomena other than incident turbulence as discussed below.

Measurement of unsteady surface pressures associated with turbulence induced lift is subject to greater uncertainty than far-field noise since surface pressure measurements are also influenced by transitional or turbulent boundary layer pressure fluctuations. The airfoil boundary layer pressure fluctuations are of high intensity but their far-field contribution is small (at unstalled airfoil conditions) due to the relatively small correlation lengths associated with such fluctuations. This was evident in previous low turbulence inflow experiments (references 10 and 11). High unsteady surface pressures were measured in these experiments when the boundary layer was turbulent and attached, but airfoil noise could not be detected above tunnel background. In the present experiment, higher levels of the 30 percent chord microphone were observed at lower test Reynolds numbers when the microphone was offset in span relative to the 15 percent chord microphone than when the two microphones were in-line. Higher levels were also observed at the 30 percent in-line position when the 15 percent chord microphone was removed and the surface faired. It was concluded that the small surface discontinuities associated with installation of the 15 percent chord microphone tripped the boundary layer from transitional to turbulent. These results indicate that pressure fluctuations associated with a fully turbulent boundary layer are lower than those obtained with a transitional boundary layer. The agreement between off-set and in-line 30 percent microphone spectra at higher test Reynolds numbers where transition would occur further forward on the airfoil tends to confirm these conclusions. Additional support is offered by the observed agreement of off-set and in-line 30 percent chord spectra at angle of attack where the adverse suction surface pressure gradient causes transition to occur at a more forward location.

The spectral cross-overs in figures $19(a)$ and (b) are believed to be caused by the presence of a laminar boundary layer at 15 percent chord and a turbulent boundary layer at other measuring stations. The high frequency regions of the plots are probably dominated by boundary layer rather than lift-associated pressure flucturations. The frequencies at which this occurs for various chordwise stations and test velocities are unknown. This demonstrates a fundamental problem in relying on blade pressure measurements to 
predict far-field noise when the phenomenon under investigation is broadband in nature rather than harmonic. In this situation, pressure fluctuations responsible for significant far-field noise are not readily separated from those associated with the boundary layer. To accomplish such separation, detailed surface pressure cross-correlations are required. In full-scale rotating blade studies, such detailed measurements are impractical.

At test velocities such as $90 \mathrm{~m} / \mathrm{sec}$ and higher, humps were noted in some of the surface pressure spectra as shown in figure 19. These are believed to be associated with airfoil vibration. While small in magnitude, airfoil vibration can affect the response of surface mounted condenser microphones. Such harmonically related vibration spikes were clearly apparent in measurements conducted at the highest test velocity of $165 \mathrm{~m} / \mathrm{sec}$.

For the lower frequencies at which far-field noise was detectable above tunnel background (figure 13) the surface spectra do show a monotonic decrease in level with increasing chord as would be expected from theory. In the next section, theoretical and experiment results are compared for the frequency ranges at which far-field noise was detectable above tunnel background noise. These ranges varied with test velocity.

Comparison of Theoretical and Experimental Spectral Results. - Shown in figure 20 is a comparison between theory and experiment for the airfoil chordwise unsteady surface pressure distribution as a function of frequency at an airfoil angle of attack of zero. The theoretical predictions shown by solid lines indicate a strong increase in unsteady pressure near the leading edge, suggesting (but not proving) that this is the dominant noise producing region of the airfoil. This strong increase near the leading edge is confirmed by the data subject to the limitation that measurement forward of 15 percent chord was precluded by inadequate airfoil thickness to house microphone instrumentation. The agreement between theory and experiment, typically within several $d B$, is considered good. considering the absolute level nature of the prediction method. The agreement is less favorable than that obtained in farfield comparisons discussed previously. The extent to which the measurement uncertainties discussed above are responsible for the less favorable agreement is not known. The significant disagreement at 30 percent chord noted at several frequencies is believed related to transitional boundary layer phenomena.

Typical Cross-Spectrum Results. - For random phenomena such as airfoil noise due to incident turbulence, prediction of airfoil far-field noise spectra from surface pressure measurements requires knowledge of the cross-power spectral density between any two points on the airfoil surface. Measurements of cross-spectra were carried out to assess the practicality of this method of calculating far-field noise. The theory applied in this study, which predicts 
far-field noise directly from the incident turbulence properties without requiring such measurements, also predicts surface cross-spectra as an intermediate step. The measurements were also taken to assess these theoretical predictions.

Since the surface pressure power spectra discussed above and surface pressure far-field cross-correlations described subsequently indicated that the leading edge region of the airfoil was the dominant noise source, spanwise cross-correlations were conducted between the two most forward instrumented locations ( 15 percent and 30 percent chord). The 15 percent chord microphone was fixed at one-third span and the 30 percent microphone was traversed in span to yield spanwise separation distances of $0,1.9,3.8,7.6,15.2$ and 22.9 $\mathrm{cm}$. Narrow bandwidth prefiltered cross-correlations were performed at each separation distance for filter center frequencies of $200,400,800$ and $1200 \mathrm{~Hz}$ and tunnel speeds of $40,60,90,120$ and $165 \mathrm{~m} / \mathrm{sec}$. Cross-correlations between microphones separated in the chordwise direction were also performed but were of less interest since the leading edge region dominated the noise generation process.

The 15 percent - 30 percent chord spanwise cross-correlations included a chordwise separation distance of $3.4 \mathrm{~cm}$. The original intent in the experiment design was to cross-correlate the 30 and 38 percent chord microphones where this chordwise separation would have been smaller $(1.83 \mathrm{~cm})$. Based on the observed strong increase in surface pressure level near the leading edge, the 15 percent location was substituted for the 38 percent chord position. Since theory is capable of predicting the cross-spectrum for arbitrary chord and span separation distances, comparison of experiment and theory was not adversely affected by the $3.4 \mathrm{~cm}$ chordwise separation. In future experiments employing blade pressure correlation data for the prediction of incident turbulence induced far-field noise, more forward locations of both microphones should be considered. As discussed subsequently, a conclusion of this study is, however, that direct prediction of noise from incident turbulence properties is to be preferred to the blade pressure measurement approach.

Figure 21 shows typical narrow bandwidth auto and cross-correlations obtained with the 15 and 30 percent chord microphones for three spanwise separation distances of $\eta=0,7.6$ and $15 \mathrm{~cm}$. The filter center frequency was $200 \mathrm{~Hz}$. Decay of the peak of the cross-correlation function (occurring near zero time delay) relative to the peaks of the autocorrelation functions (occurring at zero time delay) with increasing spanwise separation is apparent. The three correlation plots within each part of the figure were obtained with the same correlator gain and summation settings although settings vaxied among parts (a), (b), and (c). Since the zero time delay values of the autocorrelation functions are proportional to the power spectral densities at $200 \mathrm{~Hz}$ and the PSD's did not vary with $\eta$, the relative decrease of the cross-correlation 
function peaks with $\eta$ represented measurement of the decrease in spanwise cross-spectrum with increasing span. At higher frequencies the decay with $\eta$ was more rapid and the cross-correlation function could not be detected above random noise at large separation distances such as $15 \mathrm{~cm}$. The method for extracting cross-spectrum magnitude from such plots is described in more detail in Appendix $B$.

Comparison of Theoretical and Experimental Cross-Spectrum Results. - Shown in figure 22 is a comparison between theory and experiment for the airfoil surface pressure spanwise cross-spectrum as a function of frequency at an airfoil angle of attack of zero. Comparisons are given for the frequency ranges at which far-field noise was detectable above tunnel background noise. These ranges varied with velocity. Low velocity data at the two highest frequencies are not plotted since the weak cross-correlations were not detectable above random noise.

The agreement between theory and experiment is reasonable considering the absolute level nature of the predictions. The agreement is less favorable than that obtained in far-field comparisons discussed previously. The average absolute difference between the curves and data was $3.3 \mathrm{~dB}$. The theory and data confirm the expected trend of decreased spanwise correlation with increased frequency. Noise at high frequency is associated with eddies of small axial extent. Since this also means a smaller spanwise eddy length scale, the spanwise extent over which the airfoil response is correlated would be expected to be decreased accordingly. 


\title{
ADDITIONAI STUDIES
}

\author{
Correlation Studies
}

Cross-Correlation of Surface Pressures and Far-Field Noise. - In previous noise investigations with this airfoil in the UTRC Acoustic Research Tunnel, cross-correlation techniques were found to be useful in understanding fundamental noise generation processes under study. In an investigation of airfoil noise in the presence of a low turbulence inflow (reference 10), discrete frequency radiation was observed when at least one of the airfoil surface boundary layers remained laminar to the trailing edge. Cross-correlations among chordwise distributed surface pressure microphones demonstrated that the origin of the noise was the airfoil trailing edge. In another study, (reference II), surface-to-far-field rather than surface-to-surface cross-correlations were employed to demonstrate that stalled airfoil noise arises from the interaction of stall-generated eddies with the airfoil trailing edge. In the latter experiment, the delay time at the cross-correlation zero-crossing centered on the peak of the correlation region of the functions (which represents the time required for the disturbance measured by the surface microphone to manifest itself as noise in the far-field) decreased monotonically with increased chordwise position.

As shown in figure 23, significantly different behavior was observed in the present study. The figure shows typical auto and cross-correlation functions for the far-field microphone located directly above the airfoil (90 deg position) and the five surface microphones arrayed in the chordwise direction at a velocity of $120 \mathrm{~m} / \mathrm{sec}$. In the cross-correlations shown in this figure, positive delay time corresponds to delay of the surface microphone signal with respect to the far-field microphone signal. Both signals were filtered prior to correlation by a $150 \mathrm{~Hz}$ high pass filter to eliminate low frequency noise below the chamber cutoff frequency (200 Hz) associated with the tunnel fan. As shown in detail in reference $I l$ and discussed above, the zero-crossing time delay centered on the peak correlation region represents the time between a surface pressure fluctuation (cause) and its manifestation as far-field noise (effect). This results since the far-field sound is proportional to the time derivative of the lift as noted by Clark and Ribner (reference 5) in the first application of the lift-far-field cross-correlation technique.

Figure 23 shows that cross-correlations between the far-field position and all chordwise microphones display a zero crossing between 6440 and 6520 usec which is close to the calculated acoustic propagation time from the center of the airfoil to the far-field microphone of $6475 \mu \mathrm{sec}$. This demonstrated that each of the measured chordwise positions was radiating noise directIy to the far-field. Similar experimental results and this conclusion were reported 
by Fink (reference 4 ) in incident turbulence studies conducted with a flat plate. Siddon (reference 6), in prior experiments with a small disc-shaped plate ( $5.1 \mathrm{~cm} \mathrm{dia)} \mathrm{placed} \mathrm{in} \mathrm{a} \mathrm{turbulent} \mathrm{jet} \mathrm{shear} \mathrm{layer,} \mathrm{also} \mathrm{obtained} \mathrm{iden-}$ tical cross-correlation zero crossings for various surface measurement locations.

Determination of the zero-crossing time delay is subject to some uncertainty since the plotted correlation functions represent only estimates. This obtains . since the correlator input signals are random and finite averaging time is employed. For this reason it is believed that no physical significance can be attached to the small variations in zero crossing delay time among correlations conducted at various chordwise positions.

In addition to these time delay arguments, surface-to-far-field correlations can be employed to obtain quantitative information on the chordwise distribution of noise source strengths as shown by Siddon (reference 6). Starting with Curle's solution, Siddon shows, with few assumptions, that the fraction of mean square far-field sound pressure, $\overline{\mathrm{p}^{2}}$, associated with unit airfoil surface area, $d A$, is directly proportional to the slope of the surface-tofar-field cross-correlation function at the time delay corresponding to acoustic propagation from the airfoil to the far-field (i.e., at the zero crossing).

$$
\left.\overline{\frac{d p^{2}}{d A} \sim\left[\frac{\partial}{\partial \tau}\left(\overline{p_{S} p(\tau)}\right]\right.}\right]_{\tau=\frac{r}{c_{0}}}
$$

In this expression $p_{s}$ and $p$ are the surface and far-field pressures, respectively, $r$ is the surface to far-field separation distance and, $c_{0}$ is the speed of sound. This function varies with surface position and is indicative of the importance of a given elemental area in contributing to the overall noise. Siddon termed this "surface dipole strength" which is somewhat confusing in that dipole strength in unsteady lift theory is related to the local pressure jump across the airfoil and not the combined effect of local pressure jump and correlation area relevant to noise production.

Using this method, the ratio of mean square sound pressure per unit area at 30 percent, 38 percent, 50 percent and 70 percent chord to that at 15 percent chord was determined and is plotted in figure $24(\mathrm{~b})$ as a function of test velocity. Data points with leaders indicate identical values. This figure demonstrates that the dominant source of noise is the airfoil leading edge region, confirming expectations based only on surface pressure measurements. The contribution per unit area increases rapidly between 30 percent and 15 percent chord and would be expected to increase further at more forward locations where measurements were not available. The slopes shown in the correlation function plots of figure 23 do not give the appearance of following the 
above trend. However, these plots were obtained with different correlator input attenuator settings and with microphones of different sensitivity, both. of which affect the scale of the vertical axis. When properly calibrated, the slopes were found to differ by factors as large as three.

Figure $24(a)$ is a qualitatively similar plot in which the ratio of rootmean-square surface pressure at various chordwise positions to that at 15 percent chord is also shown to increase rapidly as the leading edge is approached. For incident turbulence noise, therefore, unsteady surface pressure distributions are indicative of the local noise source strength although this conclusion does not apply to phenomena such as airfoil vortex shedding discrete frequency radiation (reference 10) or stall generated noise (reference 11). The above results are in agreement with the prior findings of Siddon (reference 6) in experimentation with a small disc in a jet shear layer. Both the noise contribution per unit area and unsteady surface pressure were found to peak at the extreme upstream edge of the disk.

Siddon, in reference 6 , proceeds further to develop means of calculating correlation area and spectrum per unit area from cross-correlation information. These require experimental determination of the second derivative of far-field and surface pressure autocorrelations and a Fouriex transform of the measured correlation functions, respectively. These further calculations were not pursued here due to the difficulty of measuring these quantities with sufficient accuracy. Siddon did carry out these calculations and concluded that the correlation area was fairly invariant over the surface. This is in disagreement with the theoretical predictions of the current study as discussed in the section entitled, "Surface Pressure Theory".

The conclusion resulting from the correlation and surface pressure measurements reported here is that the leading edge region is the dominant source of airfoil noise although an equality of zero-crossing time delays demonstrated that all chordwise locations radiated directly to the far-field. This behavior is significantly different from that which obtains in the case of other isolated airfoil noise mechanisms such as discrete frequency vortex shedding and stalled flow.

Cross-Correlation of Incident Turbulence and Far-Field Noise. - To assist in understanding the incident turbulence noise generation process, a crossedwire hot wire probe was mounted $16.8 \mathrm{~cm}$ upstream of the airfoil leading edge. The probe support was normal to the sideplate on the tunnel centerline and the sensors projected approximately $15 \mathrm{~cm}$ into the stream. The wake of the thick portion of the support $(0.63 \mathrm{~cm}$ dia) therefore impinged on a spanwise section of the airfoil of length approximately $12 \mathrm{~cm}$ (or 23 percent of the total-airfoil span). This wake impingement was a potential source of additional farfield noise. This noise, however, would not affect cross-correlations between 
the hot wire and far-field noise since the hot wire sensed incident grid turbulence which is not correlated with the probe support wake turbulence.

Shown in figure 25(a) are cross-correlations between the vertical incident turbulence component and the $90 \mathrm{deg}$ far-field microphone at an airfoil angle of attack of zero. Positive time delay corresponds to delay of the hot wire signal relative to that of the far-field signal. The normalized crosscorrelation amplitudes, $\widetilde{R}$, (where hot wire and far-field zero time delay autocorrelation values have been used in the normalization) are relatively small, but measureable. Of particular interest is the dependence on time delay. Arrows indicate time delay values equal to the sum of the calculated convection time of turbulence from the hot wire to the airfoil leading edge $\left(\tau_{I}=L^{*} / U\right)$, where $I^{*}=16.8 \mathrm{~cm}$, and acoustic propagation time from the leading edge to the far-field microphone $\left(\tau_{2}=r / c_{0}\right)$. Although this calculation is approximate, first peaks in the cross-correlation functions are observed at time delays near these values. The arrows and first data peaks shift to the left with increasing velocity. corresponding to decreased convection time. This establishes cause and effect relative to the vertical turbulence component.

In figure 25(b), the axial component is observed to have no measurable correlation at zero deg angle of attack (the indicated correlation of $\widetilde{R}=0.02$ at the time delay of $11,000 \mu \mathrm{sec}$ represents measurement uncertainty as indicated by the random excursions of the other functions about zero amplitude). Such behavior would be anticipated since the axial component does not contribute to lift and is uncorrelated with respect to the vertical component. At angle of attack, however, the axial component does contribute to lift and measurable correlation is observed in the second curve of figure 25(b). While the normalized cross-correlation amplitudes are not of direct use, the time delay behavior of the functions observed in these correlation experiments is in agreement with expected trends.

Cross-Correlation of Incident Turbulence and Airfoil Surface Pressures. The hot wire probe discussed above was also employed in cross-correlation measurements with the chordwise array of surface microphones. The hot wire sensed the vertical turbulence component and the airfoil angle of attack was zero. The sensor was offset in span by a distance of $1.3 \mathrm{~cm}$ relative to the array to eliminate hot wire probe or probe support wake impingement onto the surface microphones.

Shown in figure 26(a) are hot wire cross-correlations with the 15 percent chord microphone as a function of tunnel speed. The peak correlation is observed to occur near the time delay, $\tau_{7}$, (shown by arrows) corresponding to the approximate convection time of turbulence from the hot wire to the airfoil leading edge. This is the expected result. Comparison of the correlation curves at a velocity of $40 \mathrm{~m} / \mathrm{sec}$ for a 15 percent chord location (figure 26(a)) and 50 and 70 
percent chord locations (figure 26(b)), shows that the lift response to the incident turbulence was sensed at the various chordwise positions at approximately the same time. In theory, the lift adjustment occurs at a greater time delay at aft Iocations corresponding to the propagation time of a wave from the airfoil leading edge traveling at the speed of sound in the moving stream. This propagation time was too small to be resolved in the measurements.

\section{Angle of Attack Effects}

Increase of airfoil geometric angle of attack from 0 to 8 deg was found to cause a small increase in far-field noise 90 deg data at some frequencies ( 1 or $2 \mathrm{~dB}$ ) and a somewhat larger increase in surface pressure level. Due to open jet wind tunnel aerodynamic corrections the effective angle of attack corresponding to $8 \mathrm{deg}$ was approximately $6 \mathrm{deg}$. Although a previous limited study conducted by Pater son and Hanson (reference 38 ) with a $10 \mathrm{~cm}$ chord airfoil had shown similar small angle of attack effects on far-field notse at $90 \mathrm{deg}$, increases on the order of 3 to $4 \mathrm{~dB}$ were observed at $130 \mathrm{deg}$. In the present study, angle of attack effects were within $2 \mathrm{~dB}$ at all test velocities and all angles between 70 and $130 \mathrm{deg}$. The reason for this difference is unknown. The present study indicates that angle of attack is of secondary importance. Dean (reference 9) and Clark (reference 39) also reported little or no dependence on angle of attack. Although a rigorous theoretical treatment of the angle of attack problem is not presentIy available, the theoretical considerations discussed below suggest that angle of attack effects on noise and surface pressures should be relatively small for a single airfoil.

The noise prediction discussed previously in this report was based on airfoil response functions which assume linearized flow. In this approximation, airfoil angle of attack has no effect on noise generation. Since linearized theory would be expected to provide reasonable results for small angle of attack, the effect on noise generation when the airfoil is unstalled would also be expected to be small. Accurate determination of the effect of angle of attack could be made if the airfoil response to a gust were known. At present, however, there is no theory which adequately accounts for the effects of finite angle of attack on the unsteady response of an airfoil in compressible flow.

For incompressible flow, however, the theory of Horlock (reference 40) can be applied to give some indication of the effect of finite angle of attack. Because it assumes incompressible flow, this theory is not applicable when the acoustic wavelength becomes comparable to the chord. Also, since this theory ignores the distorting effects of the flow on the turbulence, it could become inaccurate for gusts of small scale. Horlock's result shows that for incompressible flow, a finite steady angle of attack couples with a horizontal gust to give a fluctuating lift. Thus, for the parallel gust problem, in addition to the Sears lift 


$$
L_{S}=2 \pi \rho_{0} b U w_{0} S\left(\check{k}_{x}\right) e^{i \omega t}
$$

for the vertical gust, there is in addition a lift

$$
L_{H}=2 \pi \rho_{0} b U u_{0} \alpha_{S} T\left(\bar{k}_{x}\right) e^{i \omega t}
$$

due to a horizontal gust, where

$$
T\left(\bar{k}_{x}\right)=s\left(\bar{k}_{x}\right)+J_{0}\left(\bar{k}_{x}\right)+i J_{1}\left(\bar{k}_{x}\right)
$$

The total lift due to both $u$ and $w$ gusts is found by taking the sum of equations (24) and (25). Since the $u$ and $w$ gust components are statistically independent and equal for isotropic turbulence, the mean square lift required by equation (7) is given by

$$
|L|^{2}=\left(2 \pi \rho_{0} b u w_{0}\right)^{2}\left[\left|S\left(\bar{k}_{x}\right)\right|^{2}+\alpha_{s}^{2}\left|T\left(\bar{k}_{x}\right)\right|^{2}\right]
$$

The function, $T$, is of the order of 35 so that

$$
|L|^{2} \approx\left(2 \pi \rho_{0} b u w_{0}\right)^{2}\left|s\left(\bar{k}_{x}\right)\right|^{2}\left(1+9 \alpha_{s}^{2}\right)
$$

Thus, even for an angle of attack of $10 \mathrm{deg}(\sim \mathrm{I} / 6$ radian), the Horlock contribution is expected to increase the noise over that predicted by sears theory by only a factor of 1.25 , or approximately $1 \mathrm{~dB}$.

The above calculation indicates little effect of angle of attack on noise or surface pressure due to incident turbulence. Before this conclusion can be drawn conclusively, further work is needed in determining the unsteady response of an airfoil at angle of attack to a gust in compressible flow. The data, however, tend to support the conclusion that angle of attack effects are small for the case of an isolated airfoil.

Prediction of Far-Field Noise from Surface Pressure Measurements

In previous sections, good agreement with experiment has been shown in predicting both far-field noise and surface pressure statistics from measured incident turbulence properties. Since prediction of far-field noise from surface pressure statistics can be considered an intermediate step in the far-field 
calculation (the mathematical formulation can be separated into two distinct steps of: (1) turbulence to surface prediction and (2) surface to far-field prediction), prediction of far-field noise from surface pressure statistics can be considered as accomplished. Of interest here, however, is the approach that must be taken if incident turbulence statistics are unknown. In this case, limited surface pressure data must be used in conjunction with a number of assumptions to derive far-field results. The following discussion explores this approach giving an indication of the assumptions required as well as guidance in the selection of surface pressure transducer locations most useful for such surface-to-far-field prediction. For a rigorous calculation, measurement of cross-power spectral density between any two points on the airfoil surface is required. This, of course, is experimentally impractical.

The use of surface pressure measurements to predict far-field noise is actually more difficult than prediction based on turbulence characteristics and the airfoil response function. There are two main reasons for this. First, it is easier to measure the required fluctuating velocity statistics in a flow than the required surface pressure statistics. Hot wires used for velocity measurements can be placed wherever desired. Once surface microphones are fixed in the airfoil surface, however, it is difficult to move them elsewhere. Since the number of surface transducers is always limited to some extent, carefur selection of transducer locations in advance of the test is required. The second difficulty is that many more measurements are needed to charactexize the unsteady surface pressure properties adequately than are needed to characterize the incident turbulent velocity field. For velocity fluctuations, all that is needed in a far-field spectrum prediction are the turbulence intensity, length scale and the spanwi.se cross-correlation of the normal component of velocity as a function of frequency (spanwise cross-PSD). For the surface pressure method to be accurate, one needs cross-correlations as a function of frequency in both the spanwise and chordwise directions. The correlation lengths defined by spanwise and chordwise cross-correlations are a function of the chordwise position on the airfoil, as noted previously. The following gives an approximate procedure for calculating far-field noise from limited surface pressure information.

Since the loading is peaked near the leading edge as shown in figures $16(b)$ and 20 , it would be expected that the leading edge region is the most important in producing noise. Because of this, a strip theory approximation will be used to calculate the loading from the surface pressure measurements. That is, the correlated area will be assumed to be a strip of spanwise width $l_{y}^{\prime}(\omega,-b)$ (where $\ell_{y}^{\prime}(\omega,-b)$ is evaluated at the leading edge) extending over the entire chord. Because of the variation in spanwise correlation length shown in figure 16(a), which is being neglected here, this should give an underestimation of the far-field noise. However, the increase in correlation length, $\ell_{y}^{\prime}$, with chord is counterbalanced somewhat by the decrease in 
chordwise correlation with the leading edge as one moves toward the trailing edge as shown in figure 18. As can be shown from figure 16(b), the chordwise distribution of the loading at that particular frequency and Mach number is reasonably well approximated by the loading for incompressible flow, $\sqrt{(1-\bar{x}) /(1+\bar{x})}$. As found by setting $x=M \sigma$ and $y=0$ in equation (13) of reference 20 , the relation between surface loading and far-field pressure for an observer overhead of the retarded position of the airfoil (or an observer overhead of the airfoil in an acoustic tunnel) is

$$
S_{P P}(M \sigma, 0, Z, \omega)=\left(\frac{\omega}{2 \pi C_{0} Z}\right)^{2} 2 d \iiint S_{q q}\left(x_{1}, x_{2}, \eta, \omega\right) d x, d x_{2} d \eta
$$

(A factor of 4 was introduced since $\mathrm{S}_{\mathrm{qq}}$ is the square of the surface pressure rather than the pressure jump used in $\left.\frac{\mathrm{qq}}{\mathrm{r} e f e x e n c e} 20.\right)$ Making the assumption

$$
s_{q q}\left(x_{1}, x_{2}, \eta \omega\right)=\sqrt{\frac{1-\bar{x}_{1}}{1+\bar{x}_{1}}} \sqrt{\frac{1-\bar{x}_{2}}{1+\bar{x}_{2}}} S_{q q}(\eta, \omega)
$$

and using the integral

$$
\int_{-1}^{1} \sqrt{\frac{1-\xi}{1+\xi}} d \xi=\pi
$$

gives for an observer overhead of the airfoil in an acoustic tunnel

$$
S_{P P}(M \sigma, O, Z, \omega)=\left(\frac{\omega b}{C_{0} Z}\right)^{2} d l_{y}{ }^{\prime}(\omega,-b) S_{q q}(0, \omega)
$$

If the measurements made by the 30 percent microphone are used to determine the loading amplitude, $\mathrm{S}_{\mathrm{qq}}(0, \omega)$, equation (30) gives

$$
s_{q q}(-0.4,-0.4,0, \omega)=\frac{1.4}{0.6} s_{q q}(0, \omega) \approx \frac{\pi}{2} s_{q q}(0, \omega)
$$

Thus, equation (32) becomes

$$
l_{y}^{\prime}(\omega,-b)=\left(\frac{C_{0} Z}{4 b d f}\right)^{2} d \frac{S_{p p}(M \sigma, 0, Z, \omega)}{S_{q q}(-0.4,-0.4,0, \omega)}
$$


For $M=0.488$ and $f=400 \mathrm{~Hz}$, the 30 percent chord surface and $90 \mathrm{deg}$ far-field pressure levels were found to be respectively $111 \mathrm{~dB}$ and $80 \mathrm{~dB}$. Equation (34) then gives the value $\ell_{\mathrm{y}}^{\prime}(w,-b) / c=0.23$ which is comparable to the value of 0.18 show in figure $16(a)$ at $x / c=0$. This means that knowledge of the 30 percent chord surface pressure level of $111 \mathrm{~dB}$ in conjunction with a theoretical calculation of $h_{y}^{\prime}$ would predict a far-field level of 81 aB which is close to the measured $80 \mathrm{~dB}$.

It should be emphasized that equation (34) is not a general equation but is limited to the lower frequencies since the incompressible loading distribution was used. Even at these lower frequencies the result contains several approximations not necessary for the result which uses the turbulence parameters as input. The close agreement in the above sample calculation cannot, therefore, be considered an indication of the accuracy of this approach in general.

The above calculation employed measured surface pressure PSD at 30 percent chord but a theoretical calculation of correlation length. The following considers the problem of far-field noise prediction based solely upon measured surface statistics. In this approach the measured 15 percent chord - 30 percent chord spanwise cross-spectrum measurements shown in figure 22 are employed to derive a spanwise correlation length. This length is taken equal to the spanwise separation at which the cross-spectrum magnitude falls to l/e of its zero spanwise separation value $(-4.3 \mathrm{~dB})$. This length and the measured 30 percent chord PSD is then substituted into equation (34) to predict the farfield PSD at $90 \mathrm{deg}, \mathrm{S}_{\mathrm{pp}}$.

The results of this calculational procedure are compared to measured $\mathrm{S}_{\mathrm{pp}}$ values in Table II. The agreement in many cases is poor, as would be expected, based on the number of approximations involved. Accurate determination of $l_{y}^{\prime}$ from figure 22 would require about twice as many spanwise measurements. Also, the use of the 30 percent chord - 15 percent chord spanwise correlation length in equation (34) is arbitrary in that theory shows that the spanwise correlation length is a strong function of chordwise position (e.g., figure I6(a)). In addition, equation (34) assumes an incompressible chordwise loading distribution. Several estimates in Table II were reasonably close to measured values, particularly at the lower frequencies where compressibility effects are smaller. This method can therefore be employed for order of magnitude estimates. The procedure, however, is not rigorous and additional assumptions would be required to obtain directivity estimates.

Based on the above considerations several recommendations can be given relative to future use of this surface pressure statistics approach. For the incident turbulence problem, transducer locations near the leading edge are 
most important. A spanwise array (or slider capable of traverse in span) is required to define the leading edge spanwise cross-PSD. For incident isotropic turbulence, guidance in the selection of transducer spacing and maximum separation distance is offered by equation (22) for $b_{y}(\omega)$ since the surface pressure correlation length, $\ell^{\prime}$, has been shown to be the same order of magnitude. Knowledge of the longitudinal turbulence scale in equation (22) permits estimation of $\mathrm{l}_{\mathrm{y}}(w)$. Having determined this length, transducers should be spaced over a maximum distance of several $\ell_{y}(\omega)$ with a transducer spacing that provides sufficient resolution to permit integration of the cross-PSD curve to determine $l_{y}^{s}(\omega)$.

A Iimitation to this approach is that knowledge of the turbulence longitudinal scale, $\Lambda_{f}$ is required. If such detailed turbulence information were available there would be no need for surface measurements since direct prediction of far-field and surface pressures could be performed by the method described in this report. A second limitation is that the turbulence may not be isotropic in which case equation (22) cannot be applied to estimate $\ell^{\prime} \mathrm{y}^{*}$ The above comments have been concerned with the incident turbulence problem. For other sources such as stalled flow (reference 1I), discrete vortex shedding (reference 10) or the turbulent boundary layer, transducer locations at the trailing edge rather than the leading edge would be required. The above considerations indicate the complications inherent in the surface pressure transducer approach to far-field noise estimation in the general case where multiple noise generation processes associated with unsteady loading occur concurrently. 
CONCLUSIONS

1. Incident turbulence is an important airfoil broadband noise mechanism. For the approximate 4 percent turbulence intensity and 15 percent chord length scale investigated experimentally, incident turbulence noise was an order of magnitude higher in amplitude than that associated with the other isolated airfoil full-scale Reynolds number noise mechanisms of the turbulent boundary layer and stalled airfoil flow. Its relative importance in full-scale applications would depend upon the intensity and scale of the incident turbulence flow field.

2. A theory capable of absolute level prediction of airfoil far-field noise spectra, directivity characteristics, surface pressure spectra and surface cross-spectra from incident turbulence properties, without use of empirical or adjustable constants, has been validated by experimental data. Knowledge' of the spanwise cross-spectrum of the turbulence velocity component normal to the airfoil is necessary for accurate prediction.

3. The airfoil chordwise unsteady surface pressure distribution in incident turbulence is strongly peaked toward the leading edge. While all chordwise positions radiate directly to the far field, the leading edge region is the dominant source of noise.

4. The effect of angle of attack on turbulence induced far-field noise and airfoil surface pressures is small but measurable. Improvement of unsteady airfoil response theory would be required if accurate prediction of this small effect were desired.

5. Inclusion of compressibility and source noncompactness effects in the theoretical formulation is necessary to obtain accurate amplitude and directivity predictions. Finite airfoil thickness effects are important at high frequency and low velocity.

6. An existing open-jet wind tunnel shear layer refraction correction procedure appears to accurately account for refraction effects on sound propagation.

7. Direct prediction of far-field noise from inflow turbulence properties can be carried out with less uncertainty than prediction based on measurement of airfoil surface pressure properties. Direct prediction is also more desirable since it constitutes a complete rather than partial solution to the problem of noise prediction. 


\section{APPENDIX A}

\section{Discussion of Previous Investigations}

Experimental Studies. - Sharland in 1964 (reference 3) measured the noise of a small isolated flat plate $(2.5 \mathrm{~cm}$ chord by $6.4 \mathrm{~cm}$ span) placed at various axial positions in a very small $(2.5 \mathrm{~cm}$ dia) nozzle exhaust. Measurements at 8 diameters downstream in the fully turbulent jet were about $15 \mathrm{~dB}$ higher than those at one diameter where the turbulence was confined to the mixing region outside the jet potential core, thus demonstrating the importance of incident turbulence as a noise mechanism. A relatively simple noise calculation procedure employing the concept of unsteady surface pressure correlation area (not measured in the experiment) and containing a number of assumptions provided good agreement with experimental data. Incident turbulence properties were not measured. Based on the uncertainties involved in the calculation, agreement between experiment and theory must be viewed with reserve. Potter in 1968 (reference 41 ) measured the sound power of a series of $5.08 \mathrm{~cm}$ chord, $15.2 \mathrm{~cm}$ span blades immersed in the fully developed region of a jet as a function of velocity. overall power levels were predicted within 3 to $5 \mathrm{~dB}$ using Sharland's formulation. This agreement, again, should be viewed with reserve. Incident turbulence properties were not measured in Potter's investigation. Clark in 1969 (reference 39 ) measured the noise spectra of a $2.54 \mathrm{~cm}$ chord circular arc airfoil in various axial positions of a turbulent jet. The airfoil was supported by tubes although it is unclear whether these contributed to noise measured in the experiment. The vertical component of turbulence incident on the airfoil was measured, as well as autocorrelations in an attempt to categorize the turbulence length scale. A dipole far-field radiation pattern was observed. A relatively simple theory that neglected turbulence length scale was applied to predict far-field noise spectra. The agreement with data was within $5 \mathrm{~dB}$. Little dependence of noise on airfoil angle of attack was found.

Dean in 1971 (reference 9) improved on previous experiments by measuring the unsteady surface pressure at one location as well as the spectrum of the vertical component of incident turbulence. An approximate axial correlation length was calculated from the spectra. Again a free jet configuration was employed with airfoils of $3.8 \mathrm{~cm}$ chord and $7.6 \mathrm{~cm}$ span extending through the jet shear layer. A turbulence generator was used to produce a turbulence intensity of approximately 8 percent. Far-field measurements were obtained at $90 \mathrm{deg}$ relative to the upstream direction. Unsteady pressures on each surface of the airfoil were found to be $180 \mathrm{deg}$ out of phase and far-field noise independent of angle of attack. Approximate methods were applied to permit back-calculation of airfoil surface pressure correlation lengths. 
Some uncertainty existed due to the extension of the airfoil through the jet shear layer where intensities on the order of 15 percent were measured.

In reference 22, Goldstein reported previously unpublished data of Olsen giving the overall noise of a $2.8 \mathrm{~cm}$ chord strut as a function of directivity angle. The strut was located in the turbulent mixing region of a round jet. Incident turbulence properties were not measured. Theoretical prediction of overall noise level showed good agreement with experimental data although certain assumptions, discussed below, were employed. A more recent study by Olsen is reported in reference 42 . In reference 43, an approximate dipole radiation pattern was reported for an airfoil in incident turbulence.

There were several important limitations to these previous studies. First, the mean velocity and incident turbulence properties varied in the spanwise direction requiring a degree of arbitrariness in assigning a single spanwise average value for the purpose of calculation. In these studies the incident turbulence properties were either not measured or their documentation was incomplete. For example, power spectral density or axial length scale were not measured. In no case was the spanwise cross-power spectral density of the normal turbulence component measured. The extent of spanwise correlation is directly related to the far-field noise intensity as shown in this study.

Fink's recent study (reference 4) removed all of these experimental limitations with the exception that the spanwise cross-spectrum of the incident turbulence was not determined. Fink measured the noise of a $46 \mathrm{~cm}$ chord, $53 \mathrm{~cm}$ span flat plate in the presence of grid generated turbulence. Sideplates were employed as in the present study. In addition to far-field and surface pressure measurements, surface-to-far field cross-correlations were performed producing results similar to those observed in the present study.

Theoretical Studies. - There have been several previous studies which have considered the problem of the response of an airfoil to turbulence. Earlier papers dealt mainly with the problem of airfoil lift response and only recently has the problem of noise generation been attacked although the two problems are closely related.

One of the earliest papers was that of Liepmann (reference 2l). This paper laid much of the base for the treatment of airfoil-turbulence interaction problems. It was concerned with calculation of the mean square lift and based on the concept of indicial admittance functions. The indicial admittance is the response of an airfoil to a very narrow (in the spanwise direction) impulsive gust impinging on the airfoil. 
The study of Diederich (reference 44) followed along the same lines as that of Liepmann. Ribner (reference 45) studied the problem using a spectral approach in which the airfoil response to gusts varying sinusoidally in the chordwise, $x$, and spanwise, $y$, dimensions was considered. This approach is closely related to the indicial admittance method by Fourier transformations in the spanwise and time dimensions.

Because these earlier papers were concerned with airfoil lift response rather than the noise generation problem, they were limited in the extent to which they could be used to calculate noise generation. When the frequency of interest is such that the acoustic wavelength is comparable to or less than the airfoil span, noncompactness effects in the spanwise dimension preclude representing the sound by a compact dipole of strength equal to the lift for an observer not in the $\mathrm{y}=0$ plane. When the acoustic wavelength is comparable to or less than the chord, the observer must be directly above the retarded airfoil position (or nearly so) for the compact dipole representation to be used.

Some of the more recent treatments of the problem are discussed below. References 19 and 46 are concerned with calculation of the airfoil lift response for which the airfoil response function in incompressible flow are used, thus limiting the frequency range that can be considered. References 9 and 47 consider far-field sound generation, but again are limited to low frequency by the use of an airfoil response function for incompressible flow. The study by Fink (reference 4) does not have the low frequency limitation but is somewhat empirical compared to the approach used here. Kaji (reference 48), although not directly concerned with the problem of an airfoil in turbulent flow, does treat the problem of the acoustic radiation produced by the airfoil response to a discrete gust, including noncompactness effects. Goldstein (reference 22) derives results which are similar in many respects to those used here. However, by assuming compactness in the chordwise direction and using response functions for incompressible flow, that approach is limited to acoustic wavelengths which are significantly greater than a chord. 
The two-sided cross-PSD, $S_{x_{1} x_{2}}(\omega)$, of two signals $x_{1}$ and $x_{2}$ is the Fourier transform of the cross-correlation function, $R_{x_{1} x_{2}}(\tau)$

$$
S_{x_{1} x_{2}}(\omega)=\frac{1}{2 \pi} \int_{-\infty}^{+\infty} R_{x_{1} x_{2}}(\tau) e^{-i \omega \tau} d \tau
$$

which can be determined by the instrumentation arrangement show schematically in figure 27. As discussed in reference 12, the filter output in figure 27 is given by

$$
y(t)=-\frac{1}{2 \pi} \int_{-\infty}^{+\infty} x\left(t-\eta_{0}\right) h\left(\eta_{0}\right) d \eta_{0}
$$

where $h(\tau)$ is the filter impulse response related to the filter transfer function, $\mathrm{H}(\omega)$, by

$$
h(t)=\int_{-\infty}^{+\infty} H(\omega) e^{i \omega t} d \omega
$$

The correlator output $\mathrm{z}(\tau)$ is then given by

$$
\begin{aligned}
z(\tau)= & \overline{y_{1}(t) y_{2}(t+\tau)}=\frac{1}{4 \pi^{2}} \int_{-\infty}^{+\infty} d \eta_{0} \int_{-\infty}^{+\infty} d \xi_{0} x_{1}\left(t-\eta_{0}\right) h_{1}\left(\eta_{0}\right) x_{2}\left(t+\tau-\xi_{0}\right) h_{2}\left(\xi_{0}\right) \\
& =\frac{1}{4 \pi^{2}} \int_{-\infty}^{+\infty} d \eta_{0} \int_{-\infty}^{+\infty} d \xi_{0} h_{2}\left(\xi_{0}\right) \int_{-\infty}^{+\infty} H_{1}(\omega) e^{i \omega \eta_{0}} R_{x_{1} x_{2}}\left(\eta_{0}-\xi_{0}+\tau\right) d \omega \\
& =\int_{-\infty}^{+\infty}|H(\omega)|^{2} e^{i \omega \tau} s_{x_{1} x_{2}}(\omega) d \omega
\end{aligned}
$$

on the assurmption that the filters are matched (i.e., $\mathrm{H}_{7}(w)=\mathrm{H}_{2}(w)$ ). The filter bandwidth is defined as $B==_{i}^{1} \int_{-\infty}^{+\infty} d \omega|H(\omega)|^{2}$. If $s_{x_{1} x_{2}}(\omega)$ is a slowly varying function such that it can be assumed constant bandwidth, $B$, the correlator output for a filter center frequency of $\omega_{0}$ is (since $S$ is an even function) 


$$
z(\tau)=B\left[C\left(\omega_{0}\right) \cos \omega_{0 \tau}-Q\left(\omega_{0}\right) \sin \omega_{0}\right]
$$

where $\mathrm{C}$ and $\mathrm{Q}$ are the co and quad-spectrum, respectively, related to $\mathrm{S}$ by $S_{x_{7} x_{2}}=\frac{C+i Q}{2}$. The magnitude of $S_{x_{1} x_{2}}$ at frequency $\omega_{0}$ is therefore given by the peak value of the correlator plot, $z(\tau)$, divided by $2 \mathrm{~B}$.

In practice the $x_{1}$ and $x_{2}$ signal autocorrelations were obtained at each condition, in which case (for signal $x_{1}$ )

$$
z(\tau)=\int_{-\infty}^{+\infty}|H(\omega)|^{2} S_{x_{1} x_{1}}(\omega) \cos \omega \tau d \omega
$$

Evaluation at $\tau=0$ and the assumption of slorly varying functions yields the power spectral density, $\mathrm{S}_{\mathrm{x}_{1} \mathrm{x}_{1}}$

$$
z(0) \approx 2 B S_{x_{1} x_{1}}\left(\omega_{0}\right)
$$

and similarly for $\mathrm{S}_{\mathrm{x}_{2} \mathrm{x}_{2}}$. The physically realizable one-sided cross-ISD, $\mathrm{G}_{\mathrm{X}_{1} \mathrm{x}_{2}}$, or $P S D^{\prime} \mathrm{s}, \mathrm{G}_{\mathrm{X}_{1} \mathrm{X}_{1}}$ and $\mathrm{G}_{\mathrm{X}_{2} \mathrm{x}_{2}}$, are related to the two-sided functions
(reference 12 ) by $\mathrm{G}^{2}=2 \mathrm{~s}$.

By dividing the peak correlator output obtained during cross-correlation by the product of the square roots of the autocorrelation zero time delay amplitudes, a normalized cross-PSD magnitude is obtained

$$
\frac{\left|G_{x_{1} x_{2}}\right|}{\sqrt{G_{x_{1} x_{1}} G_{x_{2} x_{2}}}}
$$

This is the square root of the coherence function. Rather than operating the correlator with calibrated inputs, the above function was obtained at each condition vith input gains and attenuations set the same for the crosscorrelation and two autocorrelation measurements. Having determined this function, the absolute cross-PSD magnitude was recovered by employing the PSD's at the relevant frequency obtained from spectral analysis. The phase of the cross-PSD was given by the phase shift of the sinusoidal crosscorrelation function relative to the autocorrelation functions which peak at $\tau=0$. 
In conducting filtered cross-correlations, band-pass filters were operated in the maximally flat or Butterworth mode with both high and low pass filters tuned to the same frequency. Figure 27 shows the magnitude of the transfer function for this condition in which an insertion loss of $6 \mathrm{~dB}$ occurred at the tuned frequency and the $-3 \mathrm{~dB}$ cutoff frequencies occurred at 0.8 and 1.25 times the center frequency, $\omega$. The insertion loss canceled out in the measurement of cross-PSD since it also occurred in the PSD measurements which were used to normalize the cross-PSD function.

The filters were purchased as matched filters and the equality of their transfer functions experimentally verified. Since the filters had finite bandwidth, the measured cross-spectra represented estimates. The filter roll-off, however, was relatively sharp as shown in figure 27 . For the broadband, slowly varying spectra considered in this study, the filter bandwidth characteristics were sufficiently narrow. 


\section{APPENDIX C}

\section{Hot Wire Measurements}

Neglecting terms of order turbulence component velocity squared, the effective velocity incident on a hot wire aligned at $\pm 45 \mathrm{deg}$ to the mean velocity, $U$, is (reference 14)

$$
u_{\text {eff. }}=\frac{(\bar{U}+u \pm w)}{\sqrt{2}}
$$

For a linearized hot wire system (such as that employed here) in which the proportionality constant between voltage and velocity, $\mathrm{C}$, is the same for both wires of a crossed-wire probe, axial (u) and vertical (w) turbulence components are given by

$$
\begin{aligned}
& u=0.707 c\left(e_{A}+e_{B}\right) \\
& w=0.707 c\left(e_{A}-e_{B}\right)
\end{aligned}
$$

where $e_{A}$ and $e_{B}$ are the A.C. voltages of sensors $A$ and $B$ aligned at +45 and $-45 \mathrm{deg}$ to the free stream, respectively. A sum and difference network was employed to determine $u$ and $w$ according to equation(C2). Probe sensitivity could be set with an anemometer span adjustment. Although the recommended procedure is to align both wires perpendicular to the mean flow sequentially (reference 49) and adjust to equal sensitivity, the wake of the outer wire probe support convects onto the inner wire when the outer wire is horizontal causing significant error in not only the inner wire A.C. response but also in the D.C. response that constitutes the wire calibration. In this study, hot wire D.C. voltages were set equal with the probes in their inclined position producing zero D.C. voltage when passed through the differencing network. In this position there was no wake interference.

Axial component data were acquired with a single wire probe oriented normal to the stream. Vertical component magnitudes were determined from these data in conjunction with the relative $u$ and $w$ component magnitudes obtained in crossed-wire measurements.

A recurring experimental problem was the occurrance of spikes in the high frequency portion $(f>5000 \mathrm{~Hz}$ ) of the spectra of some hot wires. These affected, and in many cases dominated, overall rms levels. The cause was found to be vibration of the two supports that hold the hot wire in tension. These vibrations were suppressed by packing clay into the junction between the supports and the body of the probe. The importance of carefully 
monitoring the output spectra of hot wire probes during use is evident. This problem and the method of solution were previously noted by clark (reference 39). 


\section{APPENDIX D}

Derivation of the von Kármán Turbulence Functions

Referring to the coordinate geometry of the sketch below, calculation of the cross-spectrum (cross power spectral density) of the vertical component of turbulence, $w$, in the spanwise (lateral) direction, $R_{w w}\left(k_{x}, y\right)$, is required.

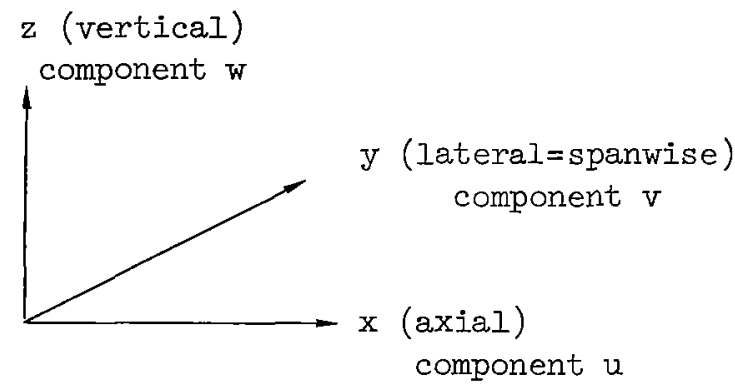

This is a function of axial wavenumber, $k_{X}$, and spanwise separation distance $\mathrm{y}$ (here $\mathrm{y}=0$ is taken as one spanwise position so that $\mathrm{y}$ equals the spanwise separation distance of the two points considered in the cross-spectrum). From equation (3-64) of Hinze (reference 14), the general expression for the energy-spectrum tensor yields for the three wavenumber spectrum of the vertical velocity component

$$
\Phi_{w w}\left(k_{x}, k_{y}, k_{z}\right)=\frac{E(k)}{4 \pi k^{2}}\left(1-\frac{k_{z}^{2}}{k^{2}}\right)
$$

Adopting the empirical von Kármán interpolation formula equation (3-130) of reference 14, the three-dimensional energy spectrum function of the turbulence, $\mathrm{E}(\mathrm{k})$, is

$$
E(k)=\left(\frac{55}{9}\right) \frac{\Gamma(5 / 6)}{\sqrt{\pi} \Gamma(1 / 3)} \frac{\overline{u^{2}}}{k_{e}} \frac{\left(\frac{k}{k_{e}}\right)^{4}}{\left[1+\left(\frac{k}{k_{e}}\right)^{2}\right]^{17 / 6}}
$$

where $k_{e}$ is the wavenumber range of energy-containing eddies. Integration of equation (DI) over wavenumber $k_{\mathrm{z}}$ then yields the two wavenumber spectrum of the vertical velocity component 


$$
\Phi_{w w}\left(k_{x}, k_{y}\right)=\int_{-\infty}^{+\infty} \Phi_{w w} d k_{z}=\frac{4 \overline{u^{2}}}{9 \pi k_{e}^{2}} \frac{\left(\hat{k}_{x}^{2}+\hat{k}_{y}^{2}\right)}{\left(1+\hat{k}_{x}^{2}+\hat{k}_{y}^{2}\right)^{7 / 3}}
$$

where $\hat{k}=\frac{k}{k}$. This is the spectrum function employed in the theory for farfield noise and unsteady surface pressure prediction. To assess the validity of the von Kármán turbulence model in this application, derivation of the PSD and cross-PSD of the vertical component is also required. The PSD of the $w$ component (one wavenumber spectrum) is found by integration of equation (D3) over wavenumber $\mathrm{k}_{\mathrm{y}}$ yielding

$$
\Phi_{w w}\left(k_{x}\right)=\frac{\overline{u^{2}} \Lambda_{f}}{2 \pi} \frac{\left(1+8 / 3 \hat{k}_{x}^{2}\right)}{\left(1+\hat{k}_{x}^{2}\right)^{11 / 6}}
$$

The spanwise cross-spectrum of $\mathrm{w}$ is given by the Fourier transform of equation (D3)

$$
\begin{aligned}
& R_{w w}\left(k_{x}, y\right)=\int_{-\infty}^{+\infty} \Phi_{w w}\left(k_{x}, k_{y}\right) e^{i k_{y} y} d k_{y} \\
& =\frac{2^{1 / 6}}{\Gamma\left(\frac{5}{6}\right)}\left(y^{*}\right)^{5 / 6}\left[k_{5 / 6}\left(y^{*}\right)-\frac{3 y^{*}}{3+8 \hat{k}_{x}^{2}} k_{1 / 6}\left(y^{*}\right)\right] R_{w w}\left(k_{x}, 0\right)
\end{aligned}
$$

where $\mathrm{y}^{*}=\mathrm{y} \mathrm{k}_{\mathrm{e}} \sqrt{1+\hat{\mathrm{k}}_{\mathrm{x}}{ }^{2}}$ and

$$
R_{w w}\left(k_{x}, 0\right)=\frac{\Lambda_{f} \overline{u^{2}}}{6 \pi} \frac{\left(3+8 \hat{k}_{x}^{2}\right)}{\left(1+\hat{k}_{x}^{2}\right)^{11 / 6}}
$$

The normalized spanwise cross-spectrum, $\widetilde{R}_{\text {ww }}$ is given by

$$
\operatorname{mim}_{w w}\left(k_{x}, y\right)=\frac{R_{w w}\left(k_{x}, y\right)}{R_{w w}\left(k_{x}, 0\right)}
$$

This cross-spectrum was previously quoted by Jackson, et al, reference 19 and Amiet reference 20. 


\section{APPENDIX E}

\section{Approximate Expressions for Far-Field Noise}

This appendix provides approximate expressions for incident turbulence far-field noise based on the full theoretical development given in the text. These expressions, while not exact, facilitate engineering calculations. Noise levels are given in terms of third-octave bandwidth Sound Pressure Level $\left(\mathrm{SPL}_{I} / 3\right)$.

The expressions apply to a directivity angle of 90 deg relative to the retarded source position. For a wing in rectilinear motion, the retarded source position is a distance aft of the present (visual) wing position given by the product of wing translation speed and acoustic propagation time from the retarded source position to the observer. The predicted spectrum applies to an observer moving with the wing translation speed. For a stationary observer, a Doppler correction is required.

The solutions can be applied directly to estimate on-axis far-field noise from a spanwise segment of a rotor since in that situation the observer is always directly above the retarded source position and there is no Doppler correction. As discussed more fully in reference (20), the solutions also apply directly to the noise produced by a stationary airfoil in the open-jet of an acoustic wind tunnel as measured by a stationary observer outside the open-jet shear layer and directly above the airfoil $\left(\phi_{\mathrm{m}}=90 \mathrm{deg}\right)$.

Two approximate expressions are given; one for high frequency and one for low frequency. The parameter which determines whether the low or high frequency expression applies is $\mu=\mathrm{MK}_{\mathrm{X}} \mathrm{b} / \beta^{2}$.

For $\mu<\frac{\pi}{4}$, the following low frequency solution applies:

$$
S P L_{\frac{1}{3}}=10 \log \left[S^{2}\left(\frac{\bar{K}_{x}}{\beta^{2}}\right) \frac{M^{6}}{\beta^{2}} \bar{K}_{x}^{2} \frac{\Delta f d}{z^{2}} \frac{\overline{u^{2}}}{U^{2}} \rho_{0}^{2} c_{0}^{4} \frac{\hat{K}_{x}^{3}}{\left(1+\hat{K}_{x}^{2}\right)^{7 / 3}}\right]+68.4
$$

where $\mathrm{S}$ is the Sears function (a function of $\overline{\mathrm{K}}_{\mathrm{X}} / \mathrm{s}^{2}$ ) which can be approximated by:

$$
S^{2}\left(\frac{\bar{K}_{x}}{\beta^{2}}\right)=\frac{1}{1+2.4 \frac{\bar{K}_{x}}{\beta^{2}}}+2 \pi \frac{\bar{K}_{x}}{\beta^{2}}
$$


The above results follow from equations (7), (11) and (12) noting also that $S_{p p}$ in equation ( 7$)$ must be multiplied by $4 \pi(0.232) f$ to convert from a one-sided $\operatorname{PSD}\left(\mathrm{S}_{\mathrm{pp}}\right)$ to third-octave bandwidth SPI.

$$
S P L_{\frac{1}{3}}=10 \log \frac{4 \pi(0.232) f S_{p p}}{\left(2 \times 10^{-4}\right)^{2}}
$$

In equation (EI), $\rho_{0}$ is in units of $\mathrm{g} / \mathrm{cm}^{3}$ and $c_{0}$ in $\mathrm{cm} / \mathrm{sec}$. Such that fSpp in equation (E3) is in units of dynes $2 / \mathrm{cm}^{4}$ appropriate to the reference mean square pressure of $\left(2 \times 10^{-4}\right)^{2}$ dynes $2 / \mathrm{cm}^{4}$. All other quantities can be in a set of consistent units since they enter as non-dimensional factors.

For higher frequencies such than $\mu>\frac{\pi}{4}$, the solution given in the text is not easily simplified. The high frequency asymptote of this solution, however, is given as equation (28) of reference 20. This equation can be generalized to include arbitrary $\rho_{0}$ and $c_{o}$ as follows

$$
S P L_{\frac{1}{3}}=10 \log _{10}\left[\frac{\Lambda_{f d}}{z^{2}} M^{5} \frac{\overline{u^{2}}}{u^{2}} \cdot \frac{\hat{K}_{x}^{3}}{\left(1+\hat{K}_{x}^{2}\right)^{7 / 3}} \rho_{0}^{2} c_{0}^{4}\right]+58.4
$$

where $\rho_{0}$ is in units of $\mathrm{g} / \mathrm{cm}^{3}$ and $c_{0}$ in $\mathrm{cm} / \mathrm{sec}$. Since equation ( $\mathrm{E} 4$ ) is the high frequency asymptote to the complete high frequency solution it is difficult to precisely define the lower frequency limit of its applicability. It can be considered an approximate solution for $\mu>\pi / 4$. 
REF'ERENCES

1. von Kármán, T. and Sears, W. R.: Airfoil Theory for Non-Uniform Motion. J. Aero. Sci., vol 5, 1938, pp. 379-390.

2. Curle, S. N.: The Influence of Solid Boundaries Upon Aerodynamic Sound. Proc. Roy. Soc. A, vol. 231, 1955, pp. 505-514.

3. Sharland, I. J.: Sources of Noise in Axial Flow Fans. J. Sound Vib., vol. 1 , no. 3, 1964, pp. 302-322.

4. Fink, M. R.: Investigation of Scrubbing and Impingement Noise. NASA $\mathrm{CR}-134762,1975$.

5. Clark, P. J. F. and Ribner, H. S.: Direct Correlation of Fluctuating Lift With Radiated Sound for an Airfoil in Turbulent Flow. J. Acoust. Soc. Amer., vol. 46, 1969, pp. 802-805.

6. Siddon, T. E.: Surface Dipole Strength by Cross-Correlation Method. J. Acoust. Soc. Am., vol. 53, no. 2 February 1973, pp. 619-633.

7. Paterson, R. W., Vogt, P. G. and Foley W. M.: Design and Development of the United Aircraft Research Laboratories Acoustic Research Tunnel. J. Aircraft, vol. 10, no. 7, 1973, pp. 427-433.

8. Foley, W. M. and Paterson, R. W.: Development of the United Technologies Research Center Acoustic Research Tunnel and Associated Test Techniques. Paper No. 7, AGARD Flight Mechanics Panel Symposium on Flight/Ground Testing Facilities Correlation (Valloire, Savoie, France), June 9-12, 1975.

9. Dean, I. W.: Broadband Noise Generated by Airfoils in Turbulent Flow. Paper 71-587, AIAA, June 1971.

10. Paterson, R. W., Vogt, P. G., Fink, M. R. and Munch, C. L.: Vortex Noise of Isolated Airfoils. J. Aircraft, vol. 10, no. 5, May 1973, pp 296-302.

11. Paterson, R. W., Amiet, R. K. and Munch, C. L.: Isolated Airfoil-Tip Vortex Interaction Noise. J. Aircraft, vol. 12, no. 1, January 1975, pp. 34-40.

12. Crandall, S. H.: Random Vibration. Vol. II, M.I.T. Press, 1963. 
REFERENCES (Cont'd)

13. Baines, W. D. and Peterson, E. G.: An Investigation of Flow Through Screens. ASME Trans., July 1951, pp. 467-480.

14. Hinze, J. O.: Turbulence. McGraw-Hill Book Co., Inc., 1959.

15. PrandtI, I.: Attaining a Steady Air Stream in Wind Tunnels. NACA TM $726,1933$.

16. Batchelor, G. K.: Theory of Homogeneous Turbulence. Cambridge University Press, 1953.

17. Compte-Bellot, G. and Corrsin S.: The Use of a Contraction to Improve the Isotropy of Grid-Generated Turbulence. J. Fluid Mech., vol. 25, part 4, 1966, pp. 657-682.

18. Paterson, R. W.: Acoustic Research Thunnel Measurements of Grid-Generated Turbulence. Report UAR-N87, United Aircraft Research Laboratories, June $14,1974$.

19. Jackson, R., Graham, J. M. R. and Maul1, D. J.: The Iift on a Wing in a Turbulent Flow. Aero. Quart., August 1973, pp. 155-166.

20. Amiet, R. K.: Acoustic Radiation from an Airfoil in a Turbulent Stream. J. Sound Vib., vol. 41, 1975, pp. 407-420.

21. Liepmann, H. W.: Extension of the Statistical Approach to Buffeting and Gust Response of Wings of Finite Span. J. Aero. Sci., vol. 22, 1955, pp. 197-200.

22. Goldstein, M. E.: Aeroacoustics, NASA SP-346, pp. 209-219, 1974.

23. Amiet, R. K.: Effects of Compressibility in Unsteady Airfoil Iift Theories. In Unsteady Aerodynamics, University of Arizona/AFOSR Symposium, March 18-20, Tucson, Arizona (ed. R. B. Kinney), pp. 631-653.

24. Amiet, R. K.: Compressibility Effects in Unsteady Thin-Airfoil Theory. AIAA Journal, vol. 12, 1974, pp. 252-255.

25. Osborne, C.: Unsteady Thin Airfoil Theory for Subsonic Flow. AIAA Journal, vol. 11, 1973, pp. 205-209.

26. Amiet, R. K.: Low Frequency Approximations in Unsteady SmaIl-Perturbation Subsonic Flows, J. Fluid Mech. To be published. 


\section{REFERENCES (Cont'd)}

27. Kemp, N. H. and Homicz, G.: Approximate Unsteady Thin-Airfoil Theory for Subsonic Flow, AIAA Journal. To be published.

28. Graham, J. M. R.: Similarity Rules for Thin Aerofoils in Nonstationary Flows, J. Fluid Mech., vol. 43, 1970, pp. 753-756.

29. Schwartzschild, K.: Die Bengung and Polarisation des Lichts Durch Einen Spalt - I, Math. Ann., vol. 55, 1901, pp. 177-247.

30. Iandahl, M.: Unsteady Transonic Flow, Pergamon Press, 1961.

31. Adamczyk, J. J.: The Passage of an Infinite Swept Airfoil Through an Oblique Gust. J. Aircraft, vol. 11, 1974, pp. 281-287.

32. Amiet, R. K.: High Frequency Thin-Airfoil Theory for Subsonic Flow, AIAA Journal. To be published.

33. Amiet, R. K.: Correction of Open Jet Wind Tunnel Measurements for Shear Layer Refraction. AIAA Paper No. 75-532, March 1975.

34. Ffowcs Williams, J. E. and Hall, I. H.: Aerodynamic Sound Generation by Turbulent Flow in the Vicinity of a Scattering Half Plane. J. Fluid Mech. vol. 40, 1970, pp. 657-670.

35. Hamming, R. W.: Numerical Methods for Scientists and Engineers. McGrawHill Book Co., Inc., 1962.

36. Amiet, R. K.: Airfoil Response to an Incompressible Skewed Gust of Small Spanwise Wavenumber. AIAA Journal. To be published.

37. Graham, J. M. R.: Lifting Surface Theory for the Problem of an Arbitrarily Yawed Sinusoidal Gust Incident on a Thin Airfoil in Incompressible Flow. Aero. Quart., vol. 21, 1970, pp. 182-198.

38. Paterson, R. W. and Hanson, D. B.: Effect of Angle of Attack on the Noise of an Airfoil in Turbulent Flow. Rep. UIRC75-35, United Technologies Research Center, May 23, 1975.

39. Clark, L. T.: Results of an Experimental Investigation Relating Sound Generation by a Single Airfoil in a Turbulent Flow. Rep. D6-23274, The Boeing Co., 1969. 
40. Horlock, J. H.: Fluctuating Lift Forces on Airfoils Moving Through Transverse and Chordwise Gusts. Trans ASME, J. Basic Eng.., Vol. 90, 1969, pp. 494-500.

41. Potter, R. C.: An Experiment to Examine the Effect of Porous Trailing Edges on the Sound Generated by Blades in an Airflow. NASA CR-66565, 1968.

42. Olsen, W.: Noise Generated by Flow Impingement on Airfoils, Cylinders, Splitters and Other Flow Obstructions; With Comparisons to Theories. ATAA Paper 76-504, July 1976.

43. Hersh, A. S. and Meecham, W. C.: Sound Directivity Pattern Radiated from Small Airfoils. J. Acoust. Soc. Amer., vol. 53, no. 2, 1973, pp. 602606 .

44. Diederich, F. W.: The Dynamic Response of a Large Airplane to Continuous Random Atmospheric Disturbances. J. Aero. Sci., vol. 23, 1956, pp. 917930.

45. Ribner, H. S.: Spectral Theory of Buffeting and Gust Response: Unification and Extension. J. Aero. Sci., Dec. 1956, pp. 1075-1077, 1118.

46. Filotas, L. T.: Theory of Airfoil Response in a Gusty Atmosphere. Part II - Response to Discrete Gusts or Continuous Turbulence. Univ. Toronto Institute Aerospace Studies, Report 141, 1969

47. Mugridge, B. D.: Sound Radiation from Airfoils in Turbulent Flow. J. Sound Vib., vol. 13, no. 3, Nov. 1970, pp. 362-363.

48. Kaji, S.: Noncompact Source Effect on the Prediction of Tone Noise from a Fan Rotor. AIAA Paper No. 75-446.

49. Bradshaw, P.: An Introduction to Turbulence and its Measurement. Pergamon Press, 1971. 
TABIE I - COMPARISON OF APPROXIMATE AND EXACT NUMERICAL LIFT RESULTS

Airfoil Lift Magnitude for Non-skewed Gust in Compressible Flow; $M=0.3^{(a)}$

\begin{tabular}{lccc}
\hline $\begin{array}{l}\text { Reduced } \\
\text { Frequency } \\
\overline{\mathrm{k}}_{\mathrm{x}}\end{array}$ & $\begin{array}{c}\text { Graham } \\
(\text { ref.28) }\end{array}$ & $\begin{array}{c}\text { Equations } \\
(11) \text { and (12) }\end{array}$ & $\begin{array}{c}\text { Equations } \\
\text { (15a) and (15b) }\end{array}$ \\
\hline
\end{tabular}

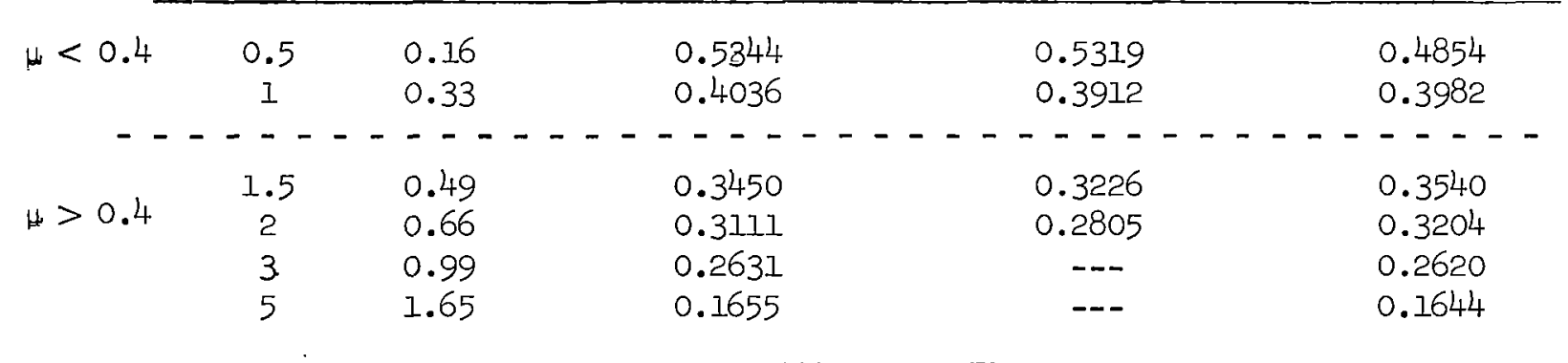

Airfoil Lift Magnitude for Skewed Gust $\left(\overline{\mathrm{k}}_{\mathrm{y}} \neq 0\right)$ in Incompressible Flow; $\overline{\mathrm{x}}_{\mathrm{x}}=\mathrm{M}=\mathrm{O}^{(\mathrm{b})}$

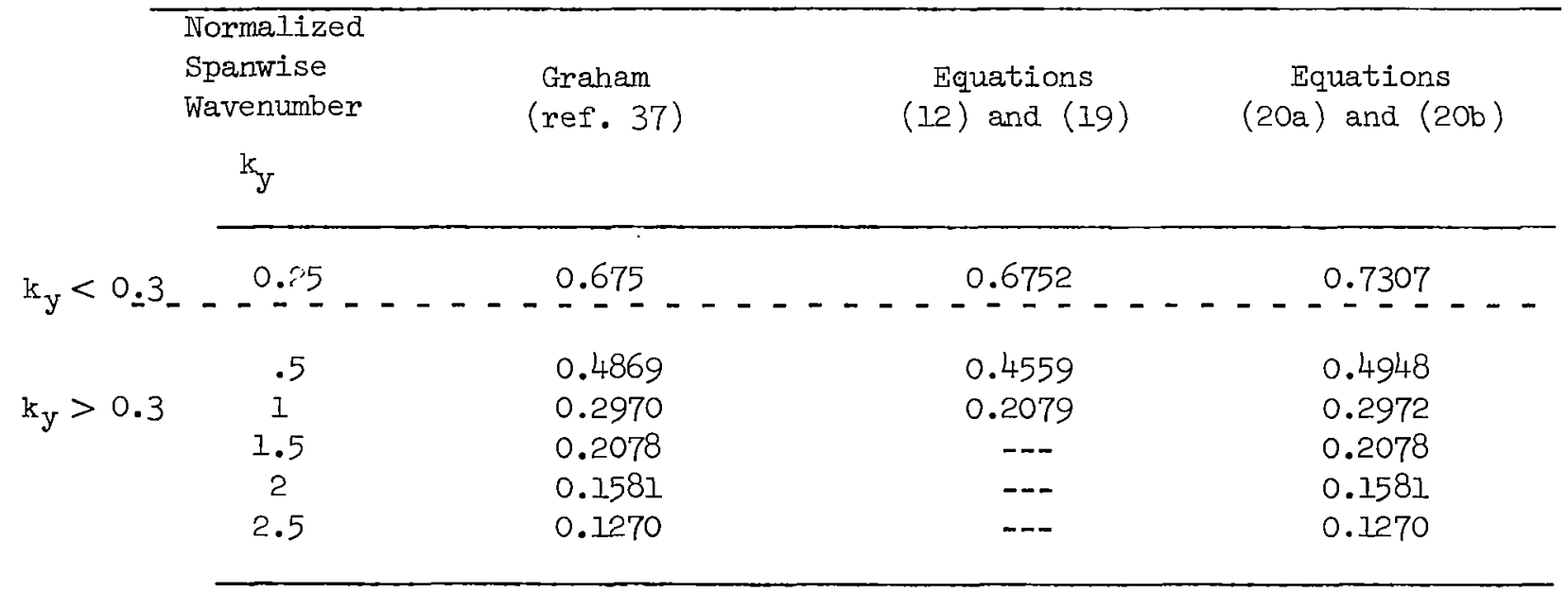

(a) Lift for approximate theories found by setting $x=M \sigma$ in expression for $\mathcal{L}$

(b) Lift for approximate theories found by integration of expressions for pressure distribution; or see references 31 and 32 . 
TABIE II

ESTIMATED 90 DEGREE NOISE BASED ON SURFACE PRESSURE MEASUREMENTS

\begin{tabular}{|cccc|}
\hline $\begin{array}{c}\text { Velocity, } \\
\text { m/sec }\end{array}$ & $\begin{array}{c}\text { Frequency, } \\
\mathrm{Hz}\end{array}$ & $\begin{array}{c}\text { Measured } \\
\text { Spp, dB }\end{array}$ & $\begin{array}{c}\text { Estimated } \\
\text { Spp, dB }\end{array}$ \\
\hline \hline 40 & 400 & 52.5 & 52 \\
& 800 & 51.5 & 55.5 \\
& 1200 & 41.5 & 52 \\
\hline 60 & 200 & 60 & 60.5 \\
& 400 & 63 & 61 \\
& 800 & 56 & 61 \\
& 1200 & 47 & 60 \\
\hline 90 & 400 & 71 & 68.5 \\
& 800 & 67 & 68.5 \\
& 1200 & 60 & 67.5 \\
\hline 120 & 400 & 76 & 76.5 \\
& 800 & 74 & 75 \\
& 1200 & 68 & 74.5 \\
\hline 165 & 800 & 80 & 84 \\
& 1200 & 76.5 & 84 \\
\hline
\end{tabular}



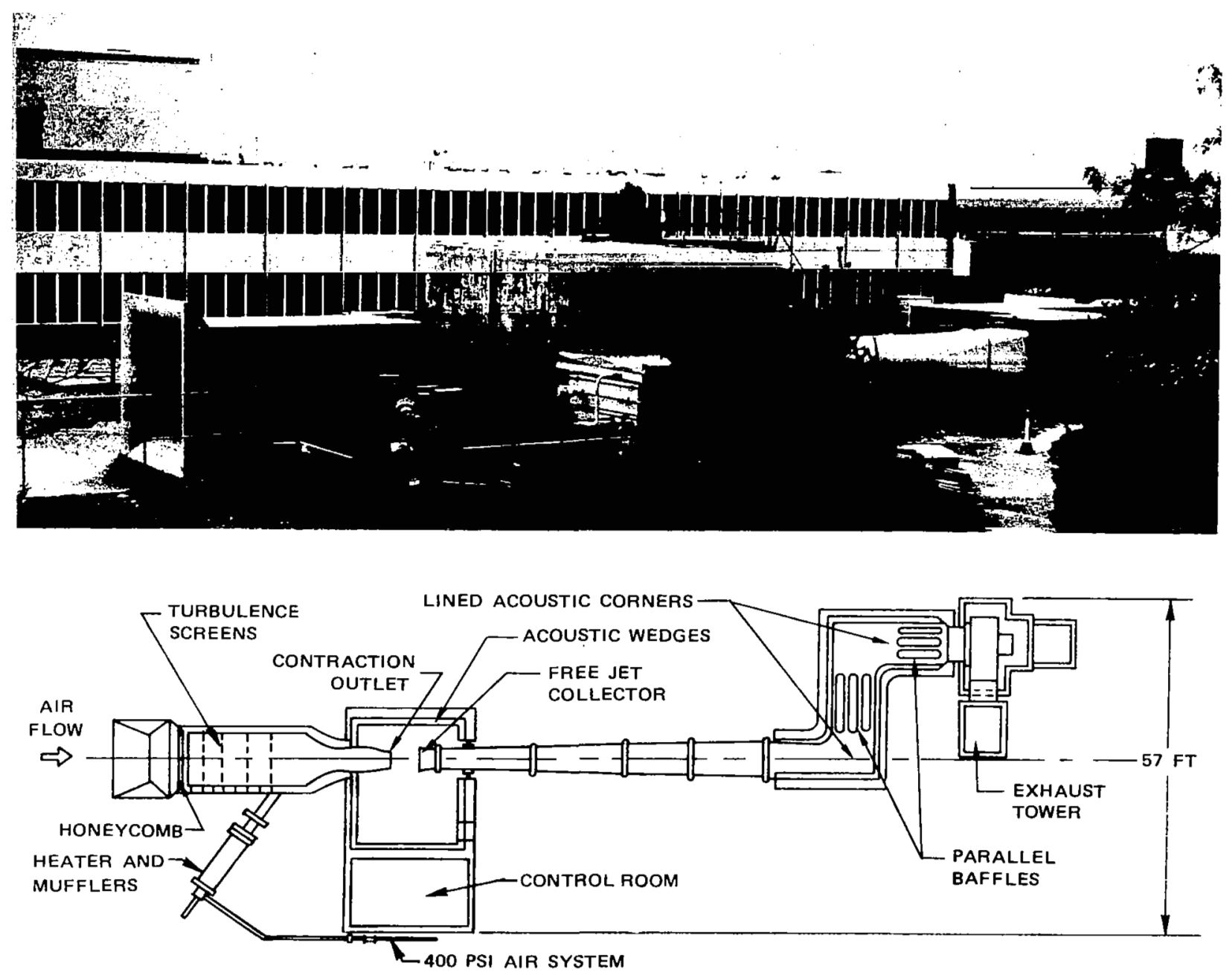

TOP VIEW

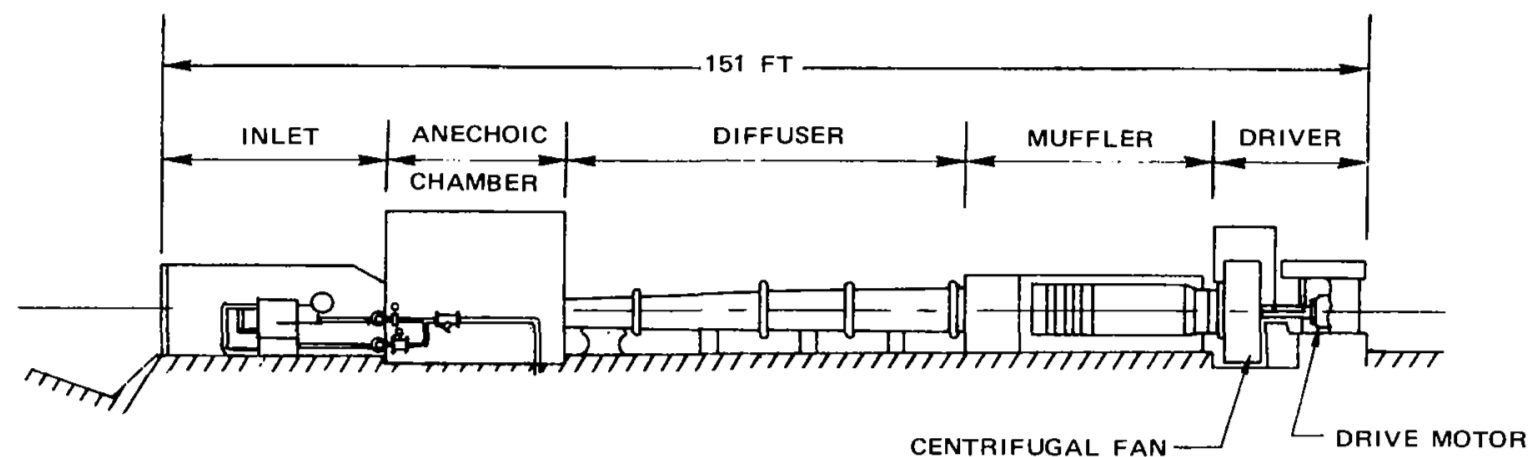

SIDE VIEW

FIG. 1 - UTRC ACOUSTIC RESEARCH TUNNEL 


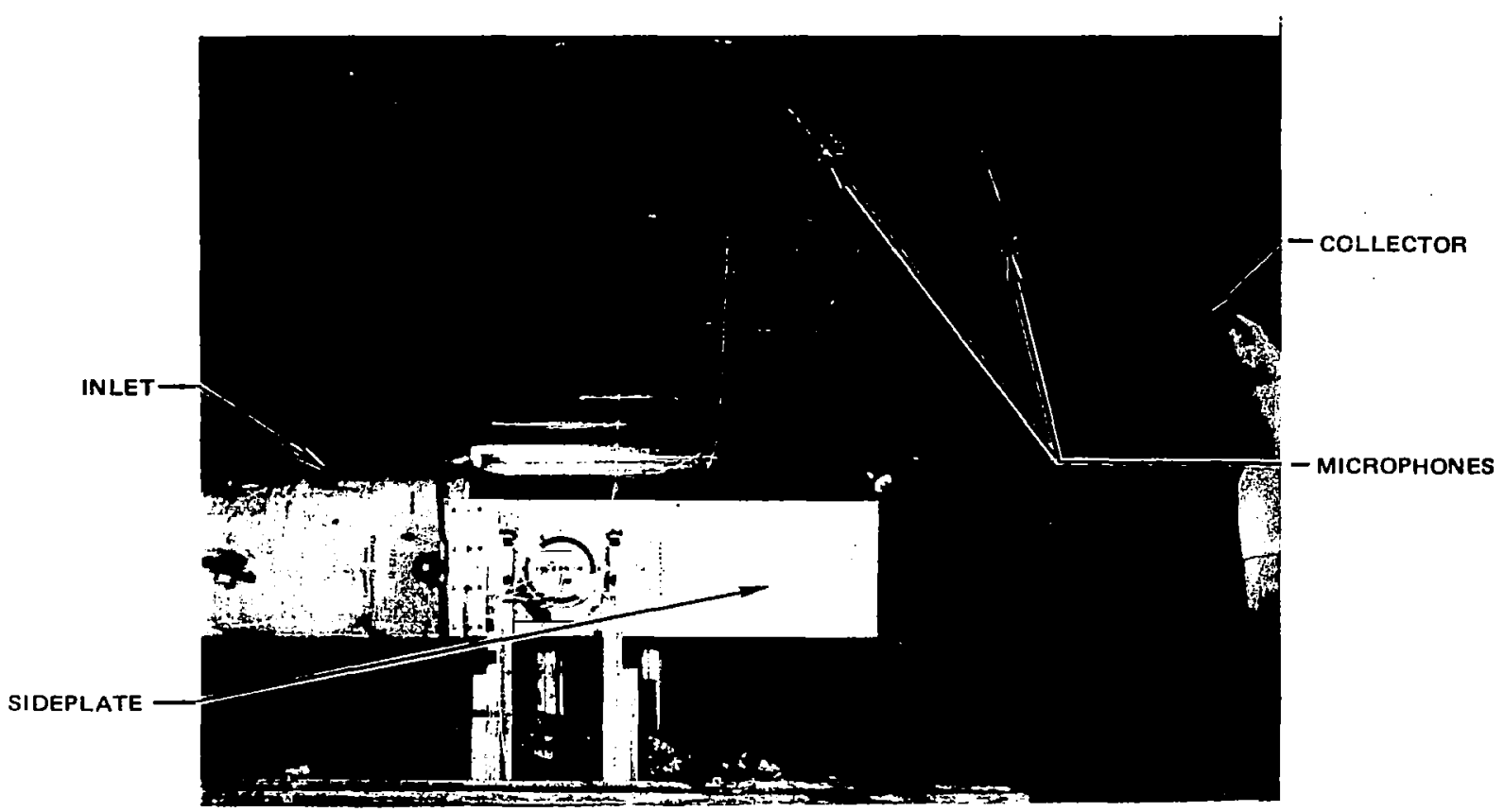

(a) SIDE ELEVATION

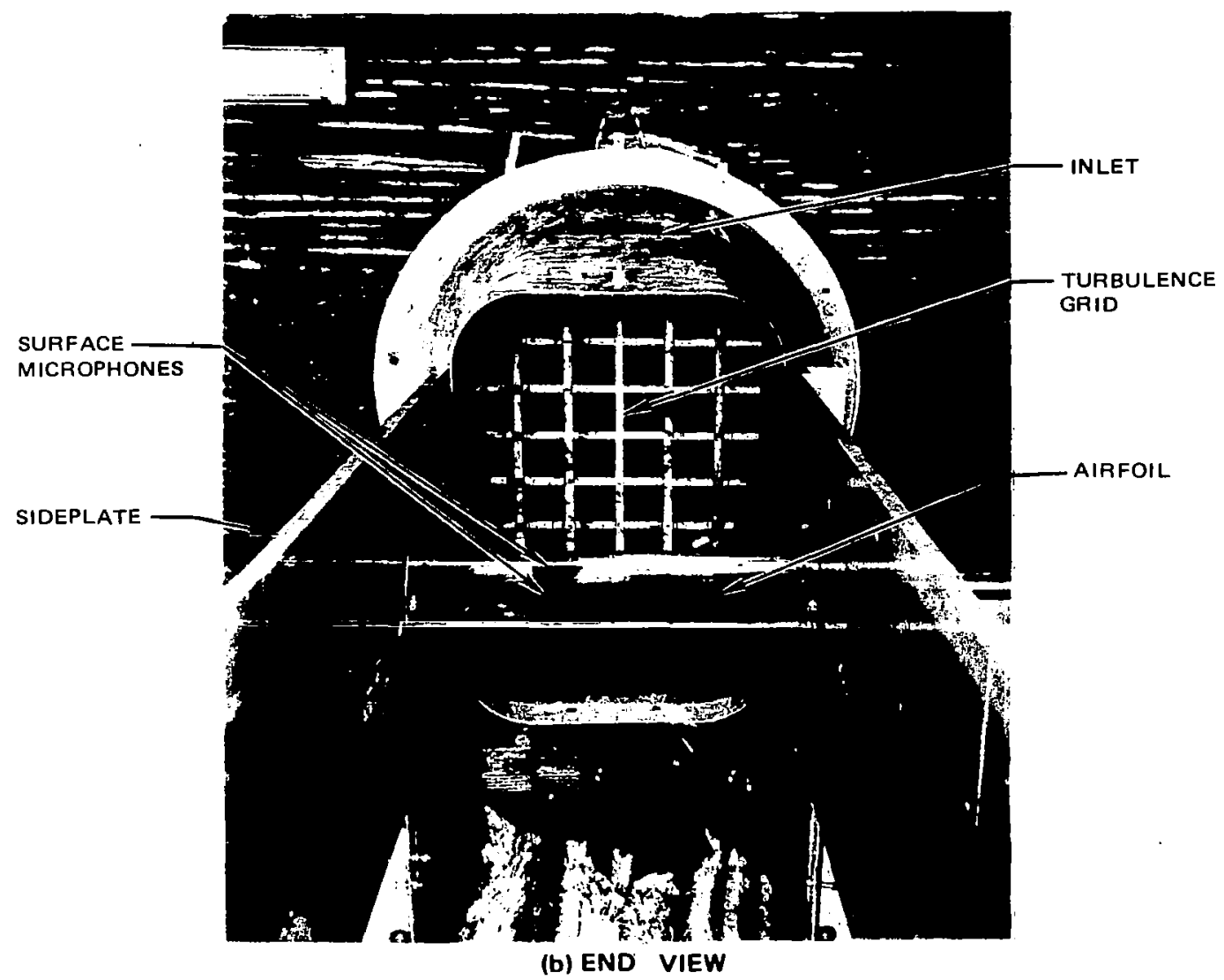

FIG. 2 - TEST SECTION ARRANGEMENT 
(8) $40 \mathrm{~m} / \mathrm{sec}$

(b) $60 \mathrm{~m} / \mathrm{sec}$

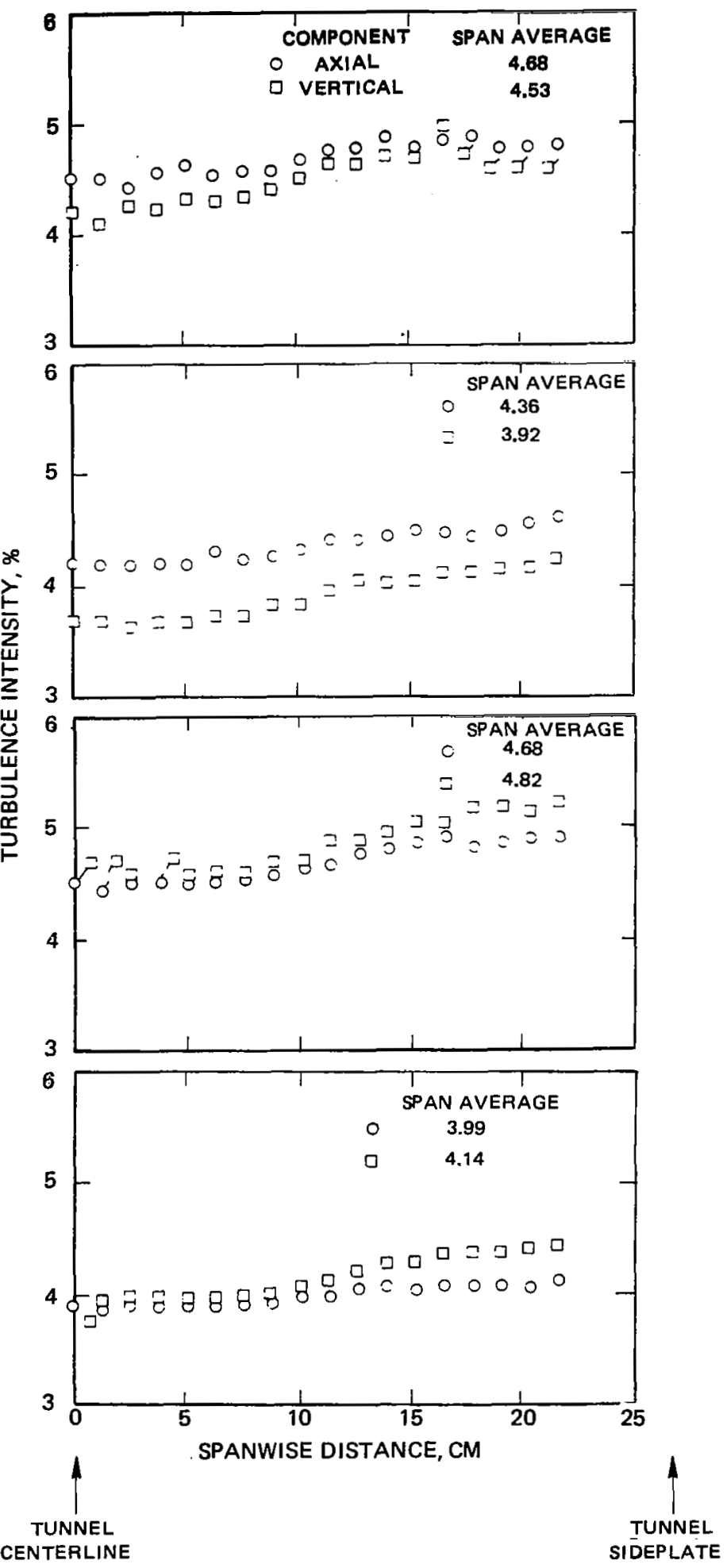

FIGURE 3 - SPANWISE DISTRIBUTION OF TURBULENCE COMPONENT INTENSITIES 
(a) AXIAL COMPONENT

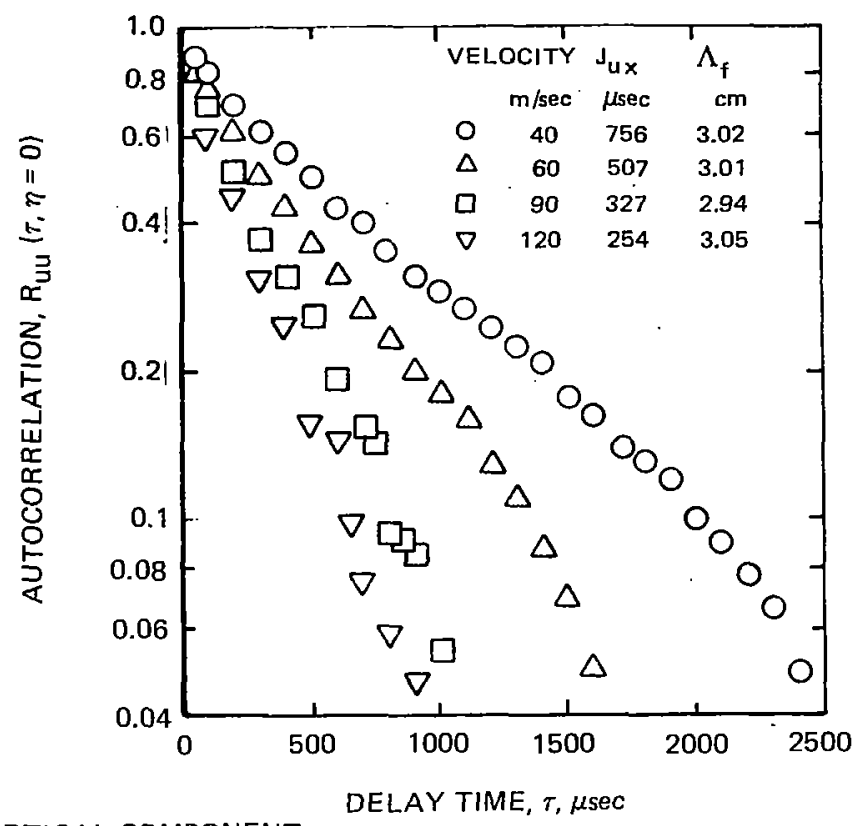

(b) VERTICAL COMPONENT

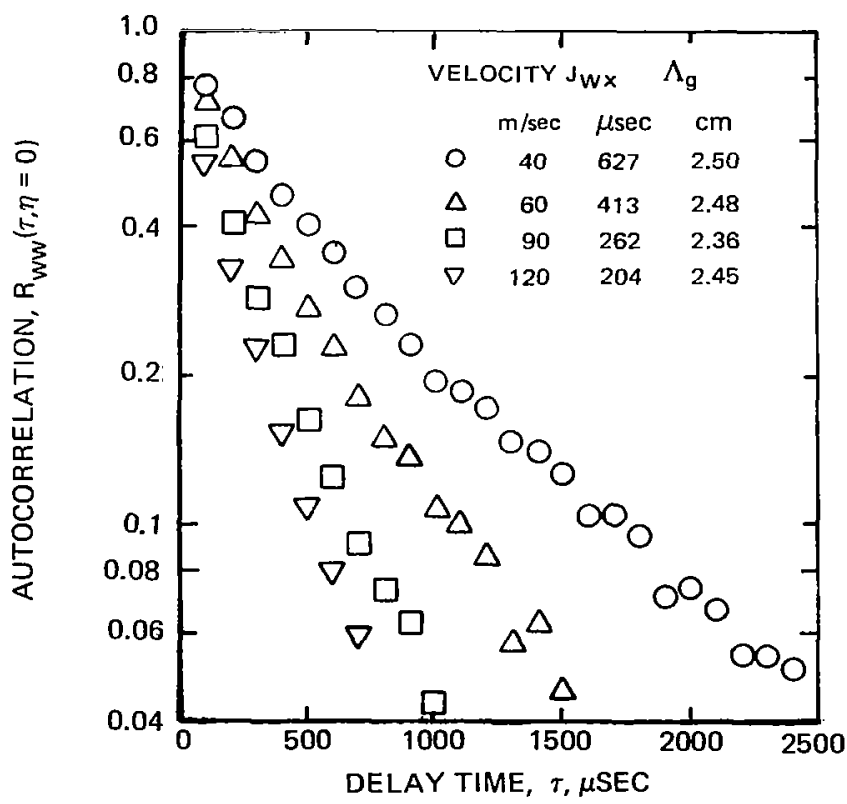

FIGURE 4. - TURBULENCE COMPONENT AUTOCORRELATIONS 

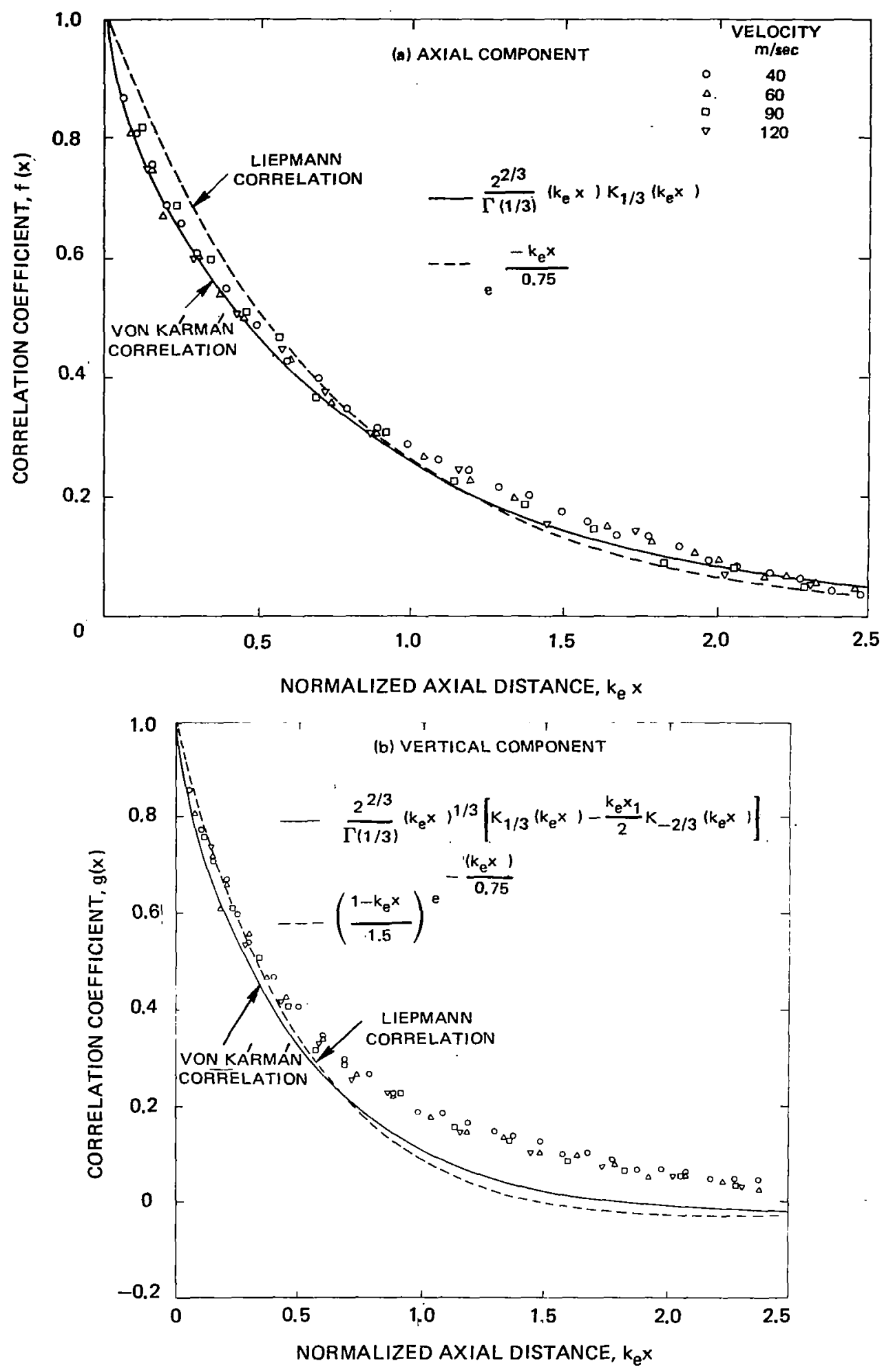

FIGURE 5. - AXIAL CORRELATION OF AXIAL AND VERTICAL TURBULENCE COMPONENTS 

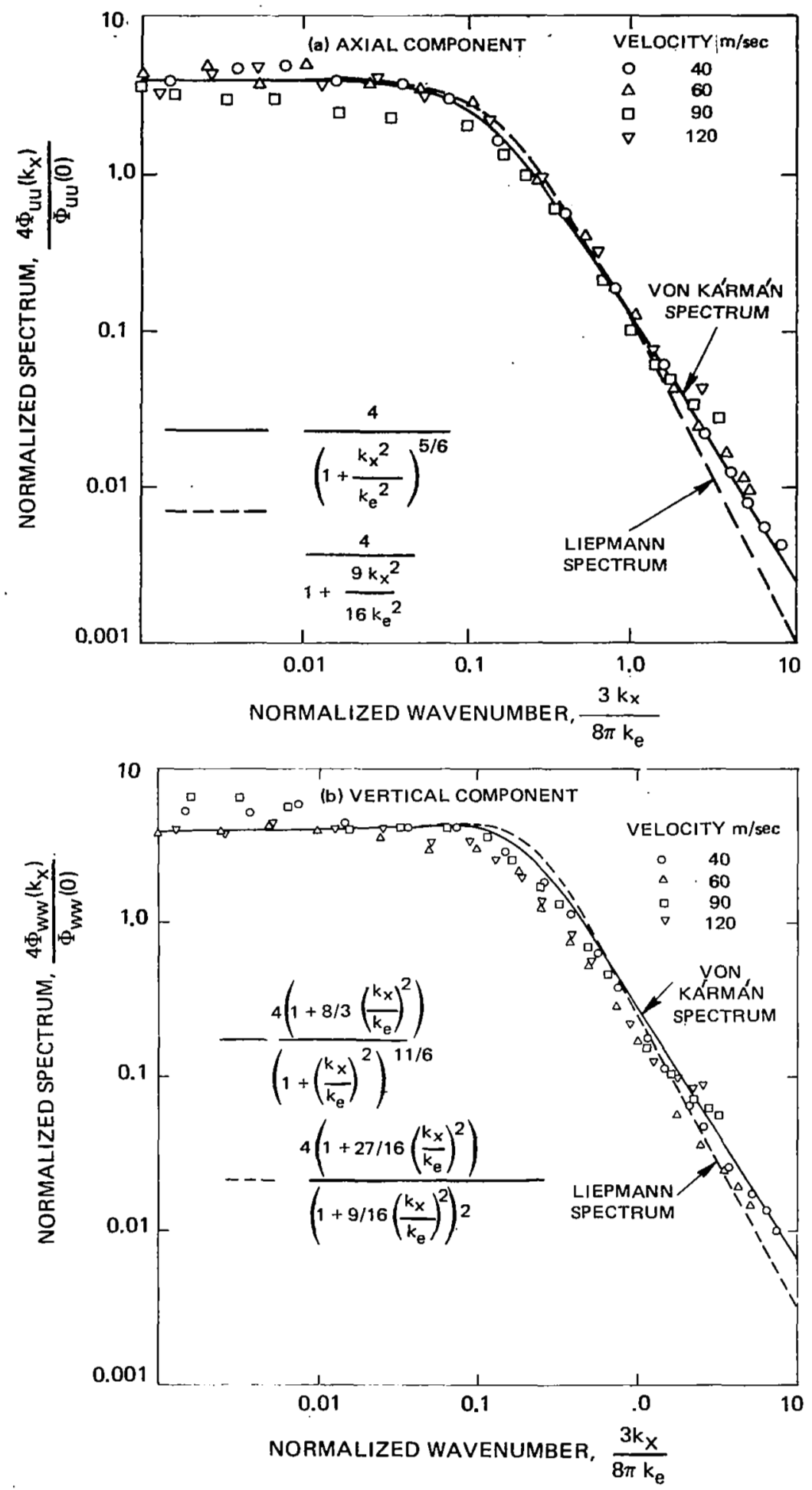

FIGURE 6. - ONE-DIMENSIONAL SPECTRUM OF AXIAL AND VERTICAL TURBULENCE COMPONENTS 

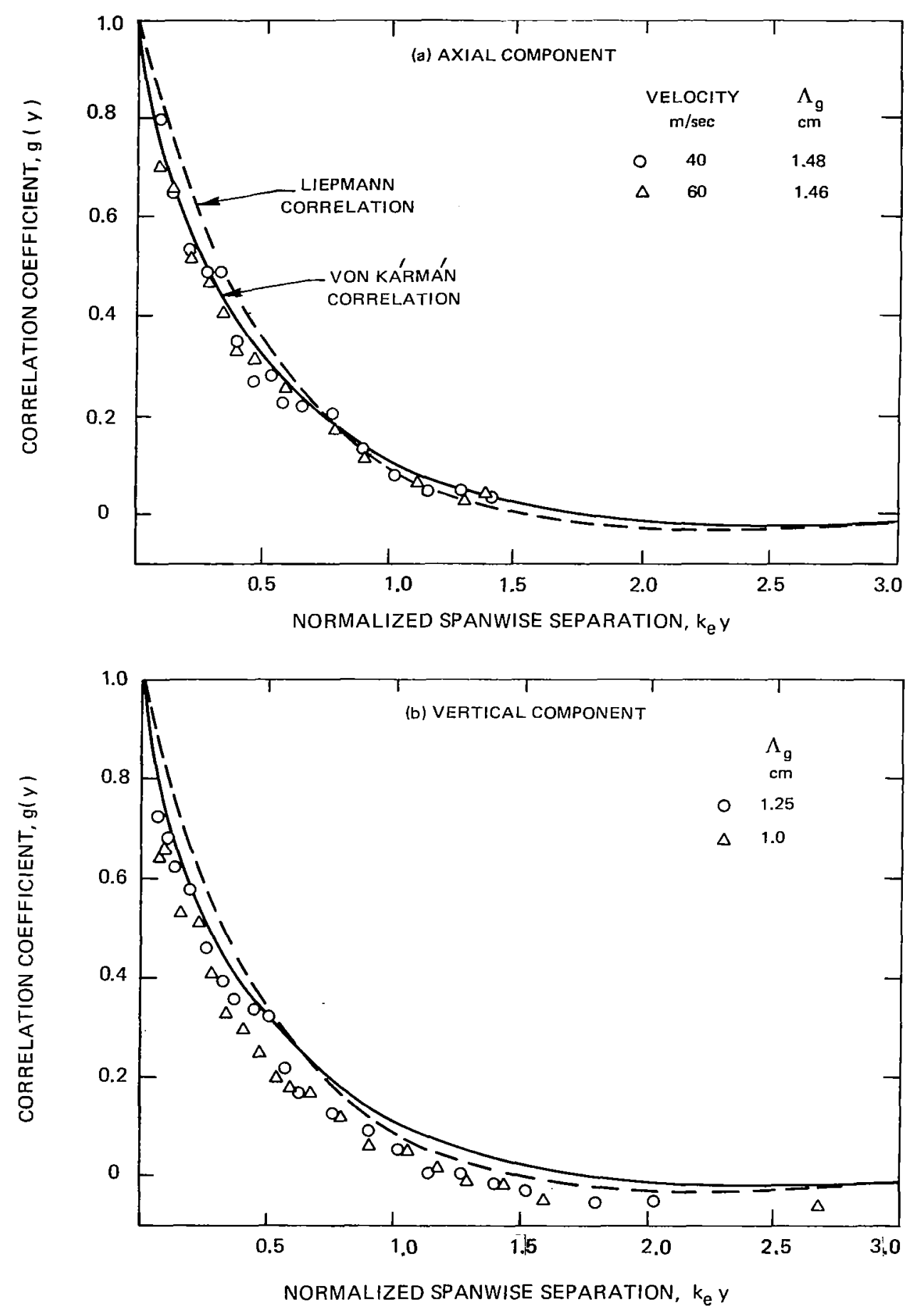

FIGURE 7.- SPANWISE CROSS-CORRELATION OF AXIAL AND VERTICAL TURBULENCE COMPONENTS 


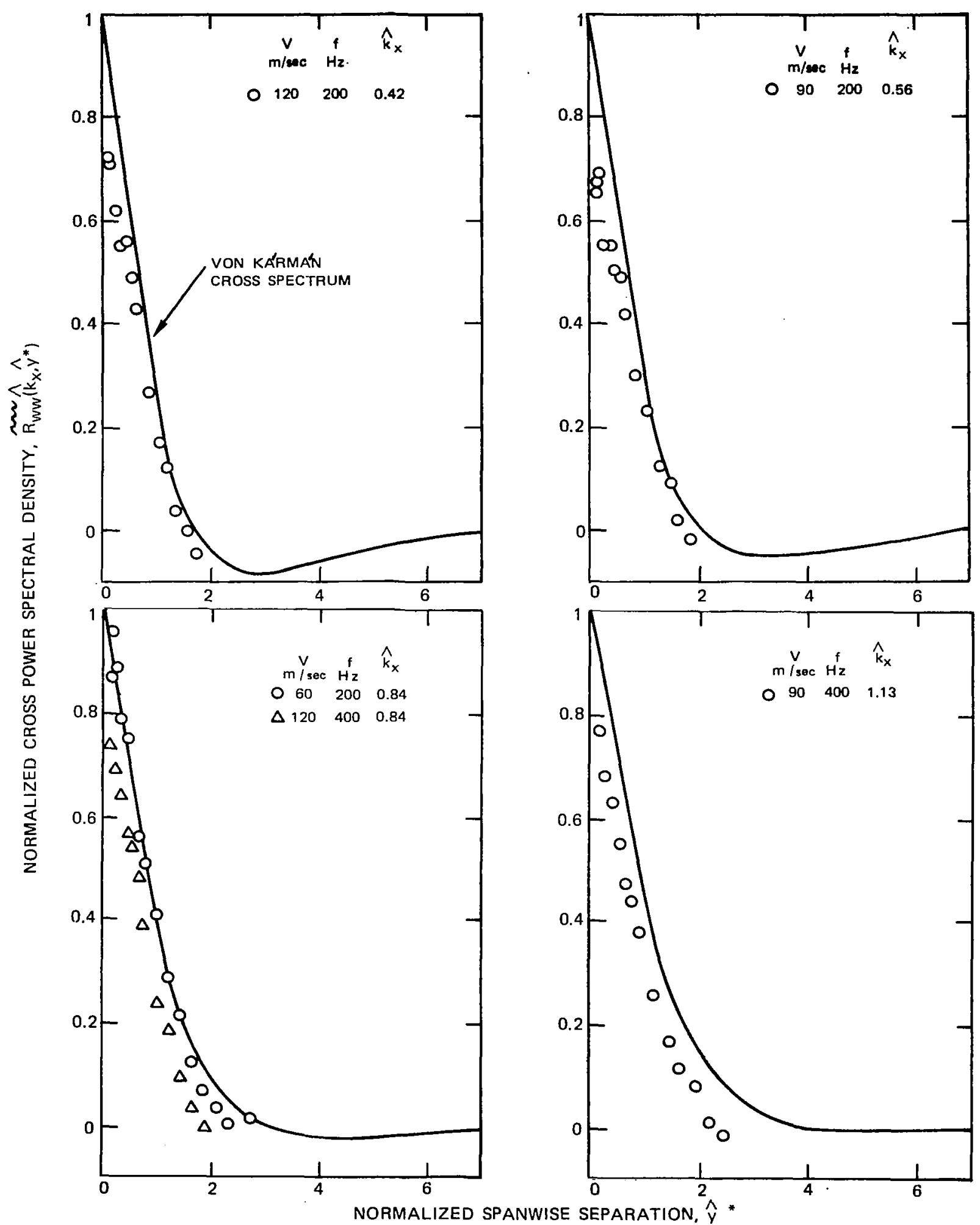

FIGURE 8 - SPANWISE CROSS-SPECTRUM OF VERTICAL TURBULENT VELOCITY COMPONENT 

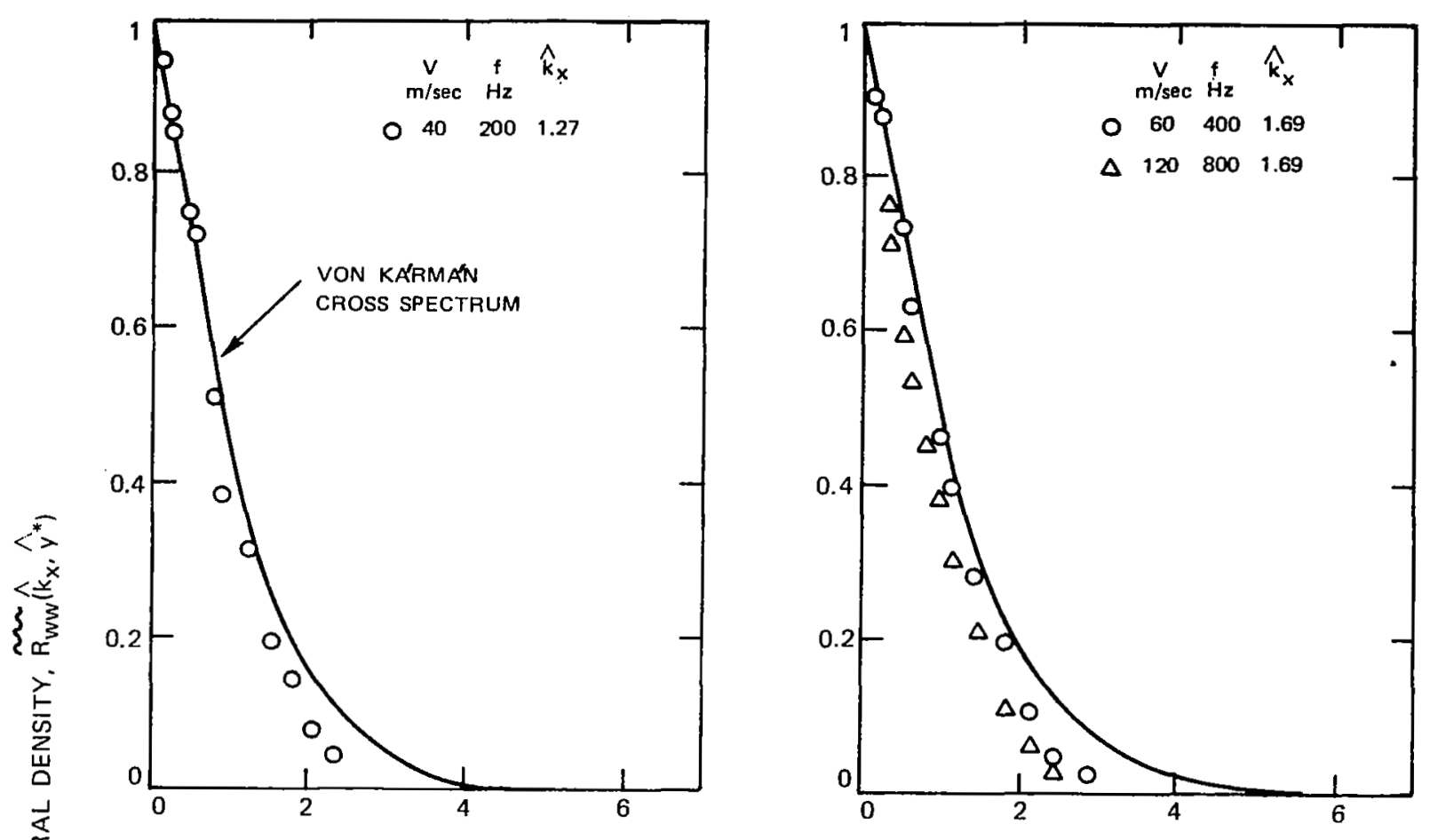

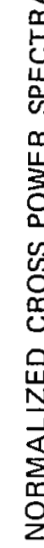

证
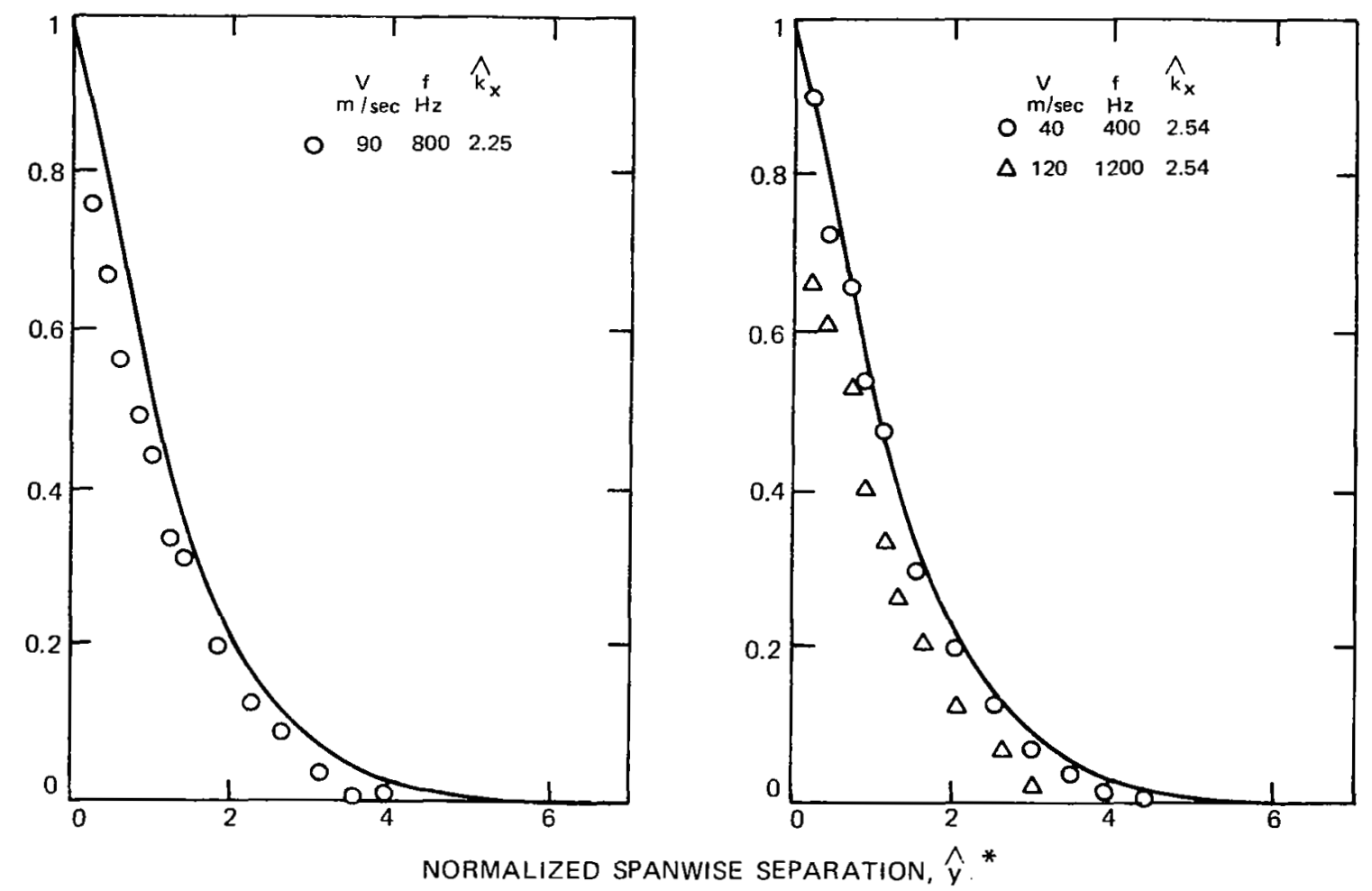

FIGURE 8 - CONTINUED 

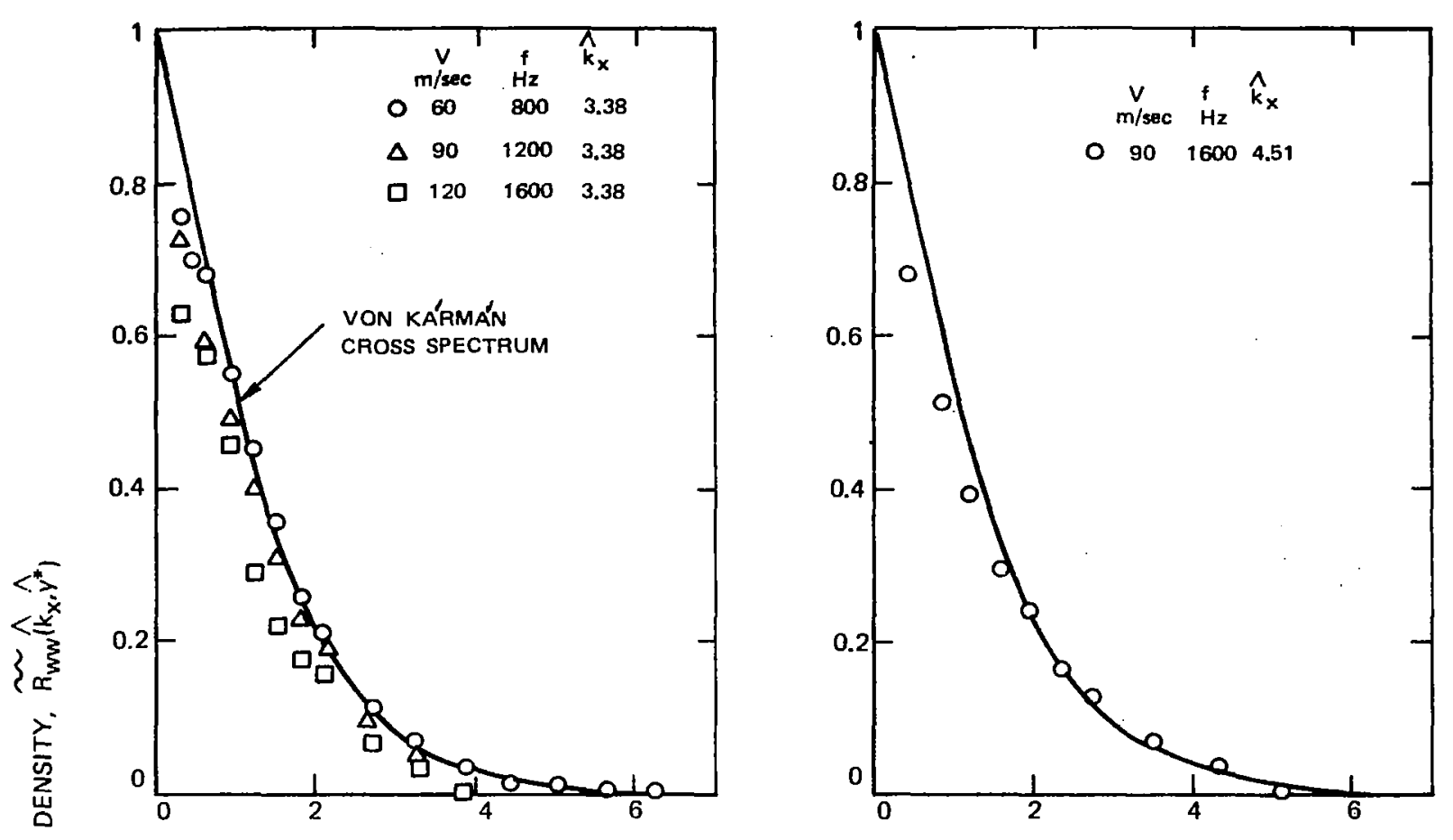

年
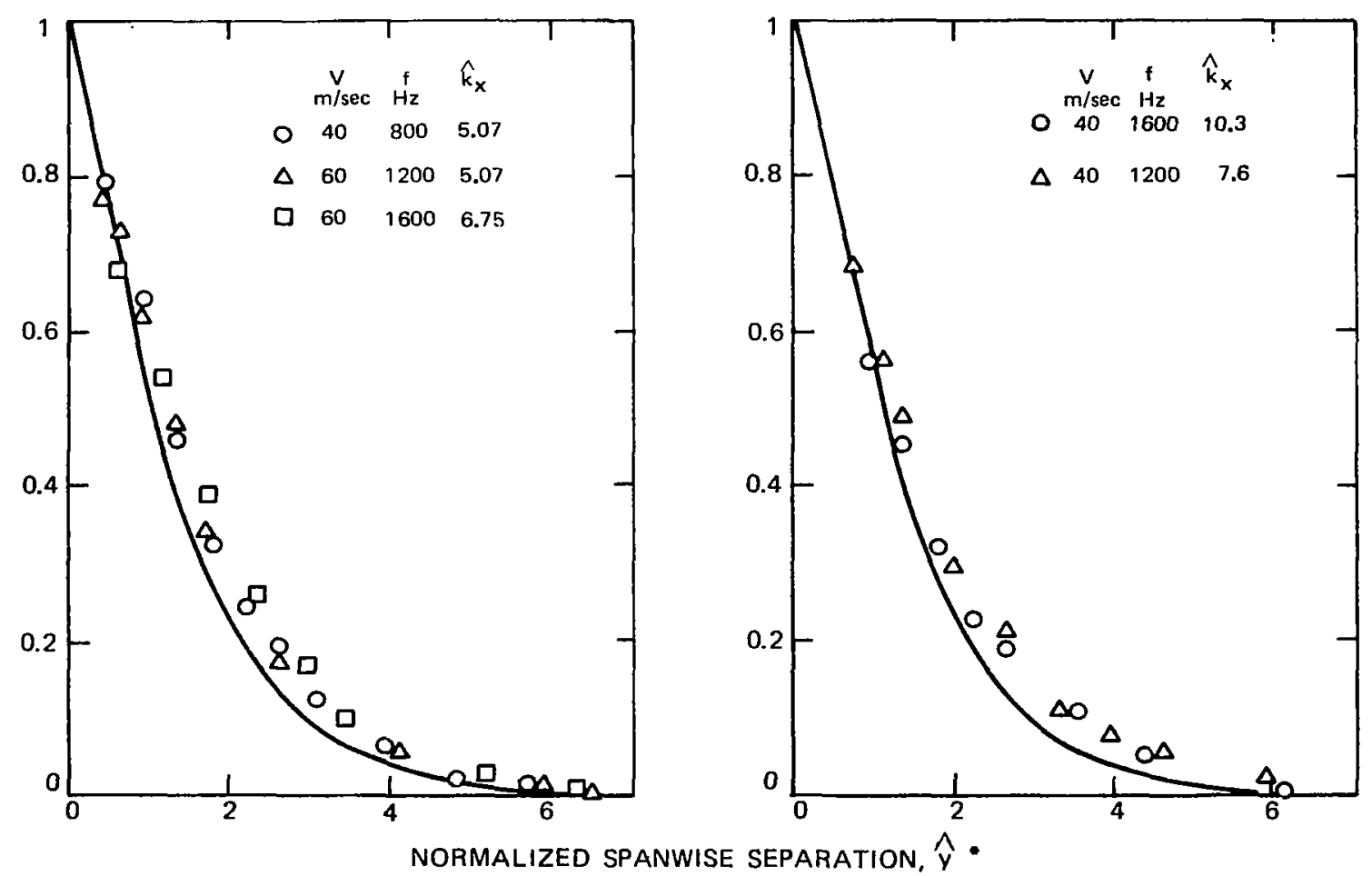

FIGURE 8 - CONCLUDED 


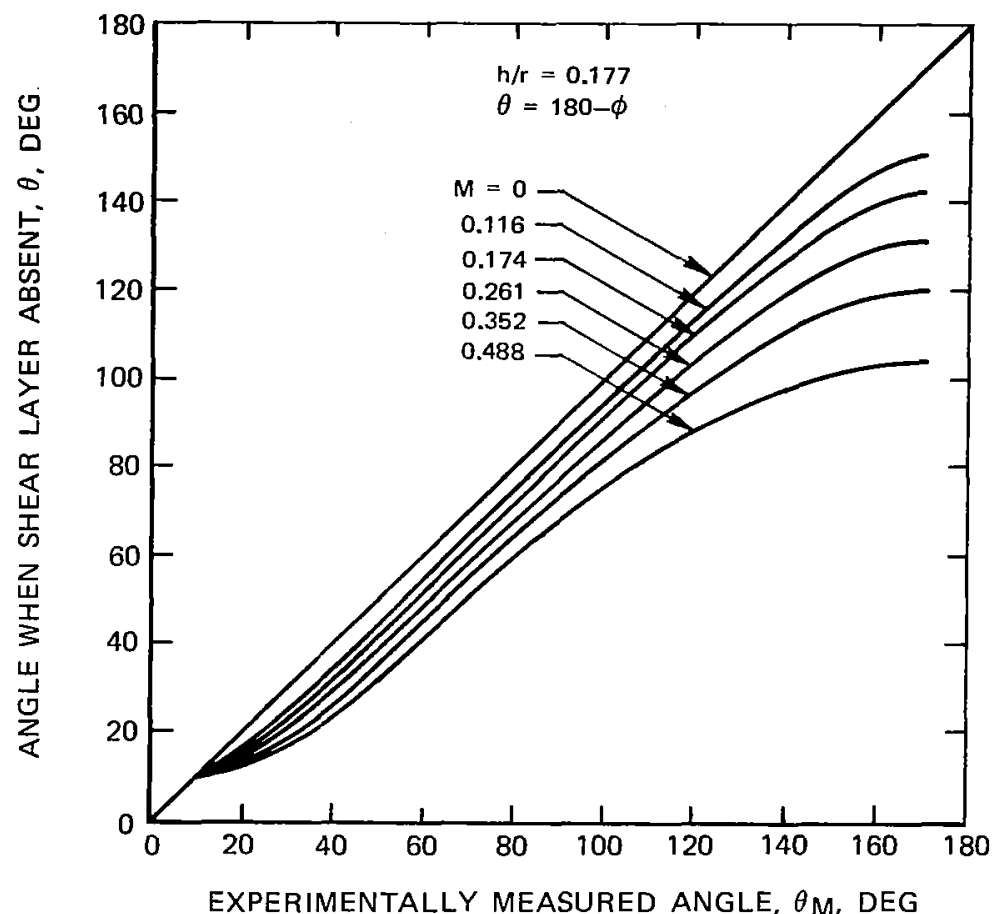

(a) ANGLE CORRECTION

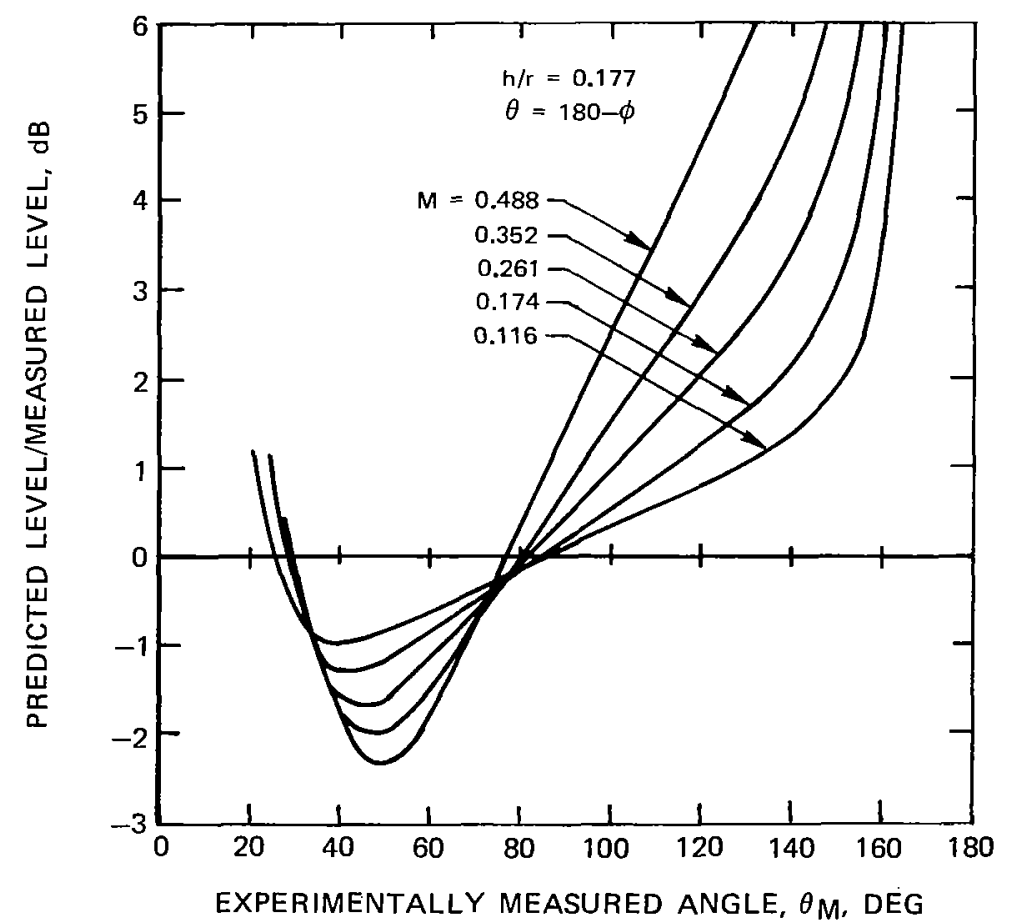

(b) AMPLITUDE CORRECTION

FIGURE 9. - SHEAR LAYER ANGLE AND AMPLITUDE CORRECTIONS 


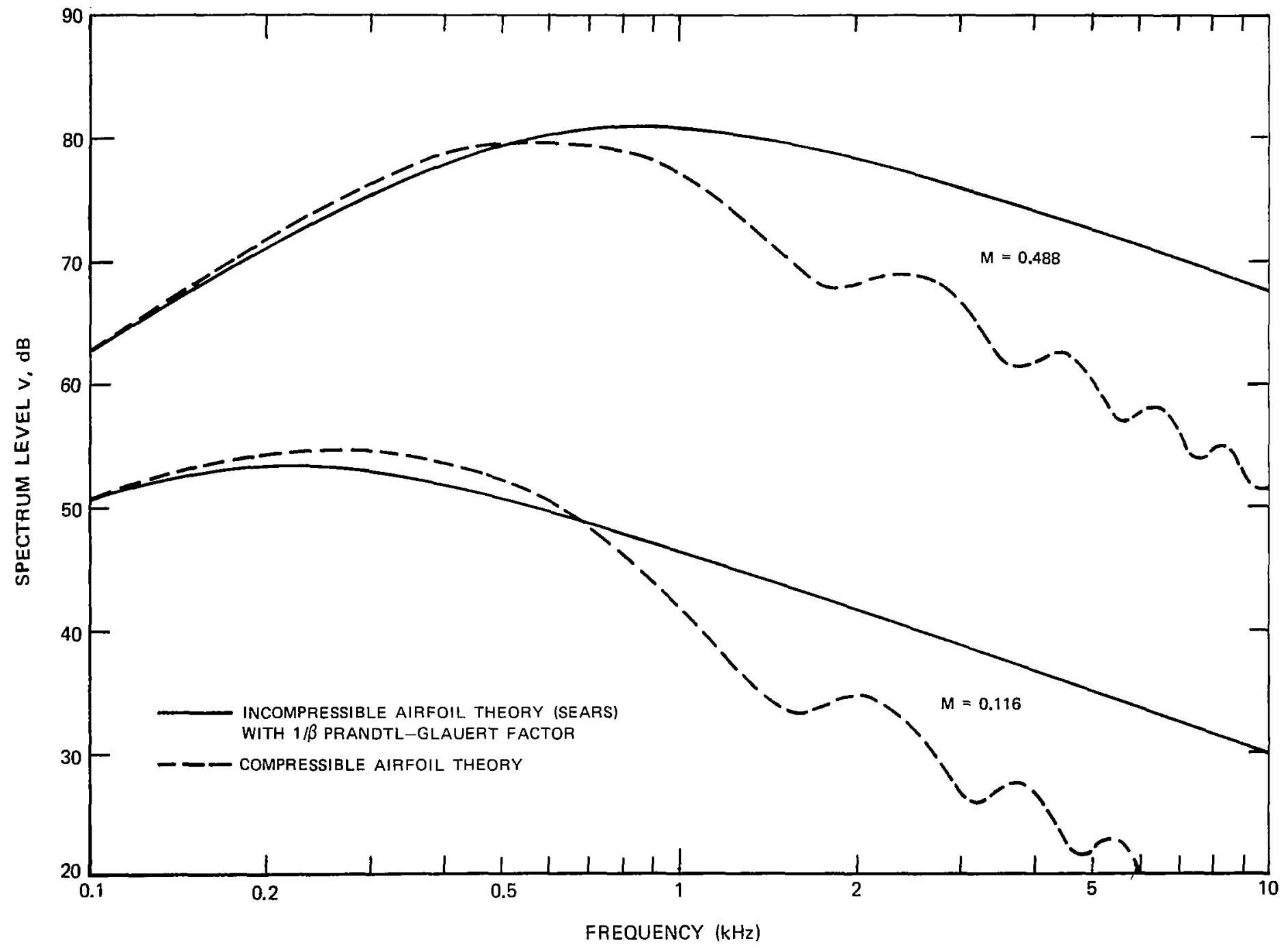

FIGURE 10. - FAR-FIELD NOISE CALCULATED USING COMPRESSIBLE AND INCOMPRESSIBLE AIRFOIL RESPONSE FUNCTIONS 


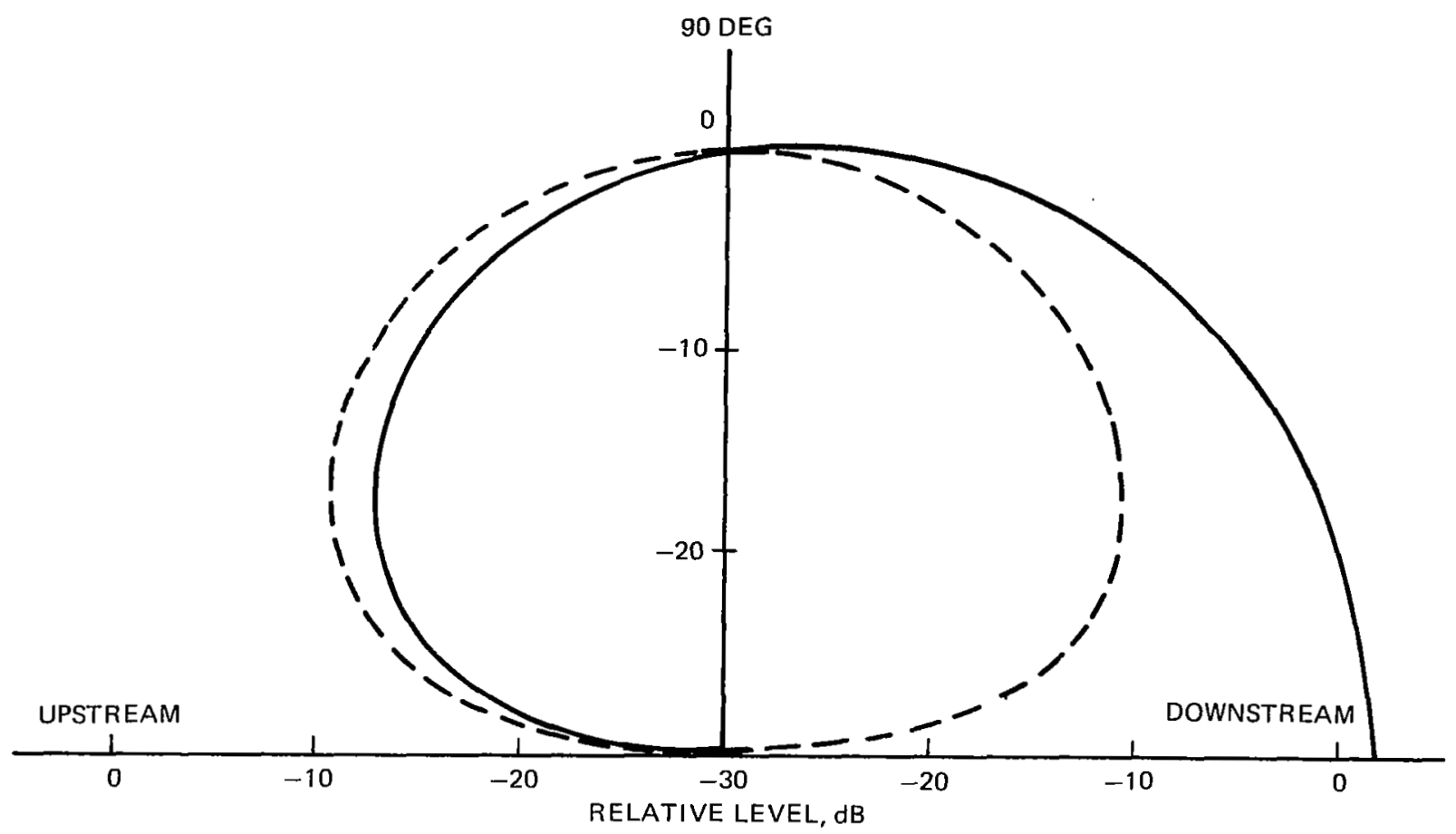

- EDGE NOISE; HIGH FREQUENCY LIMIT

- - COMPACT DIPOLE

FIGURE 11. - ZDGE NOISE DIRECTIVITY COMPARED WITH THAT OF COMPACT DIPOLE IN A UNIFORM STREAM OF MACH NO. $M=0.488$. 


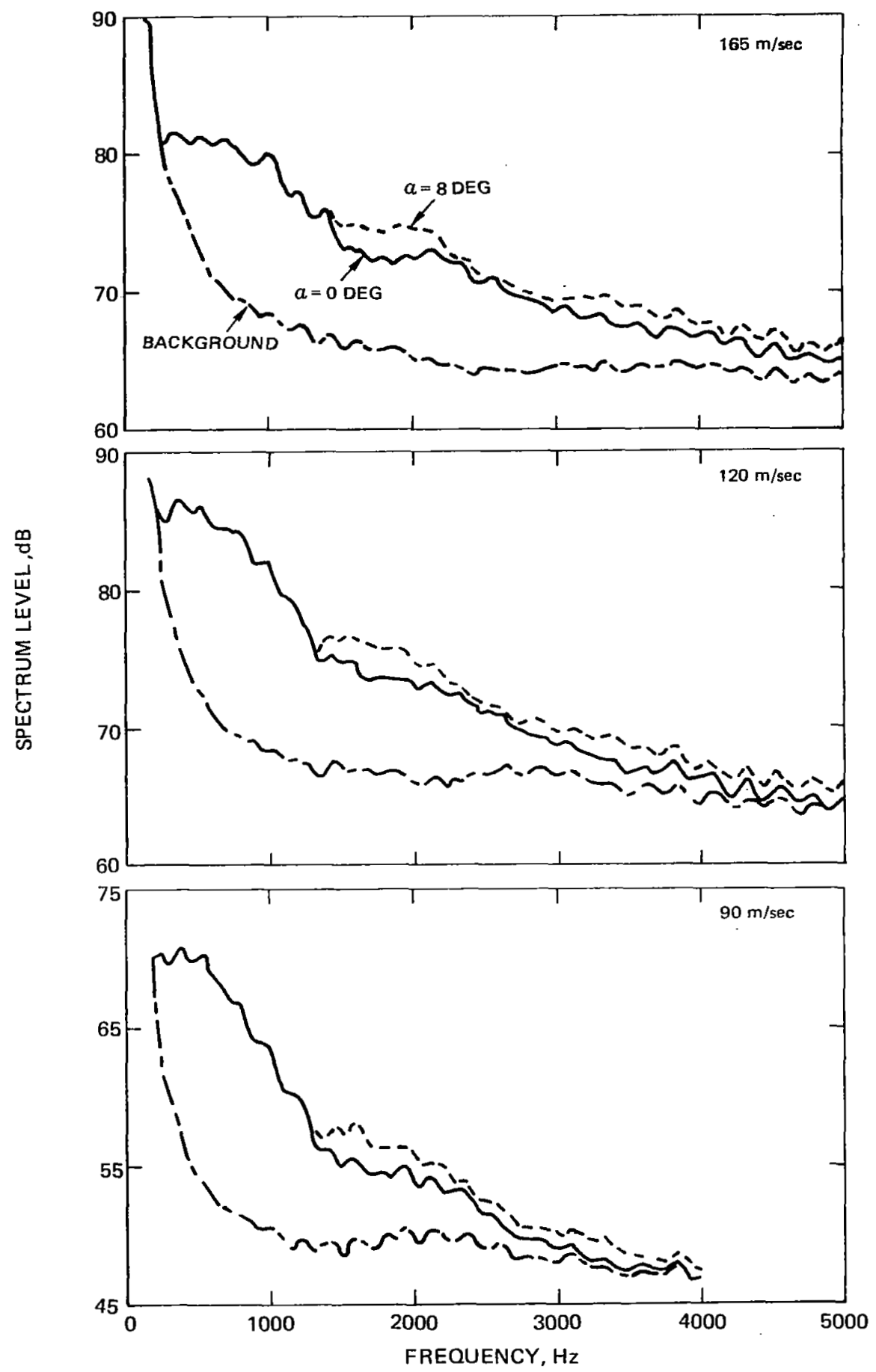

FIGURE 12. - FAR-FIELD NOISE SPECTRA AT $\phi=90$ DEG 


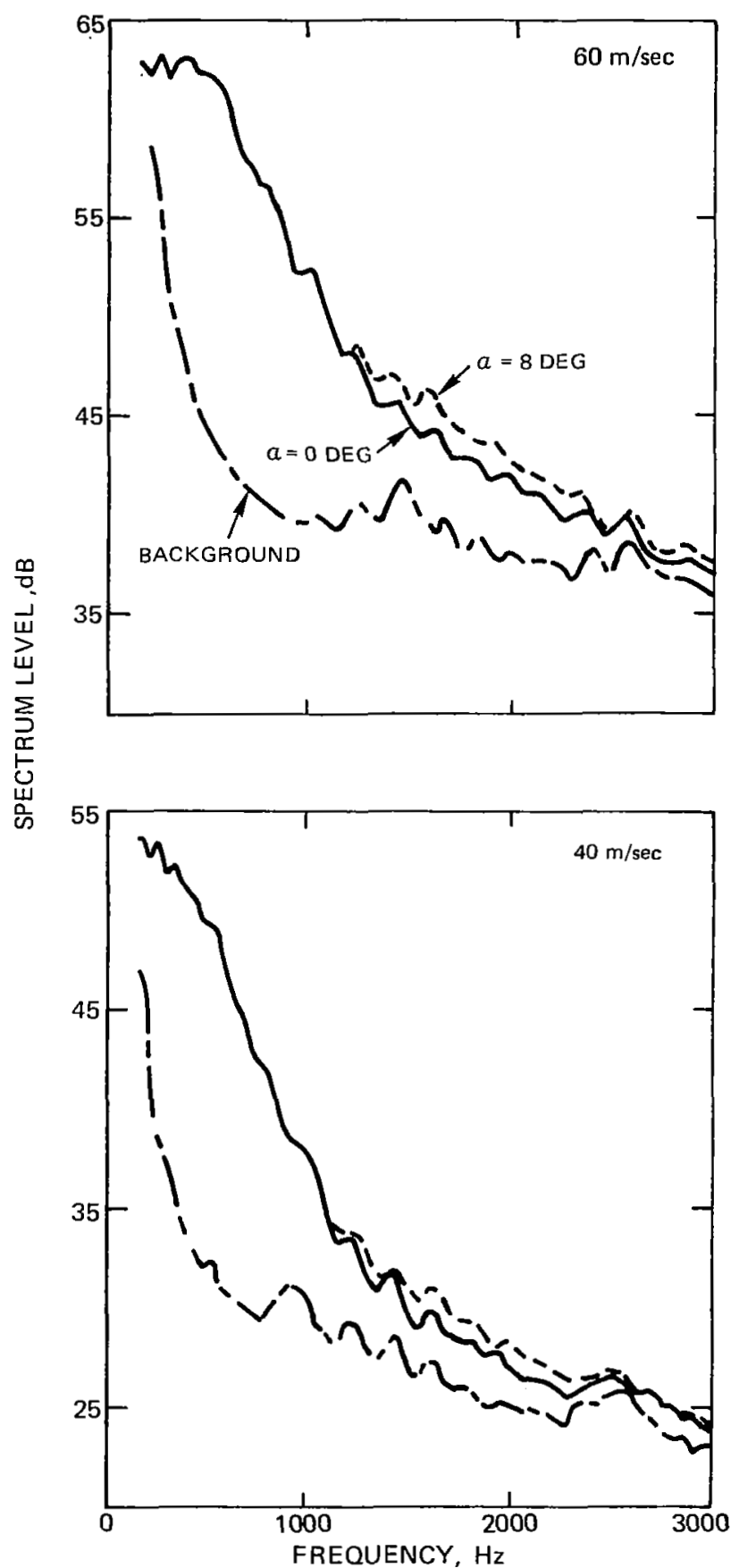

FIGURE 12. - CONCLUDED 
|

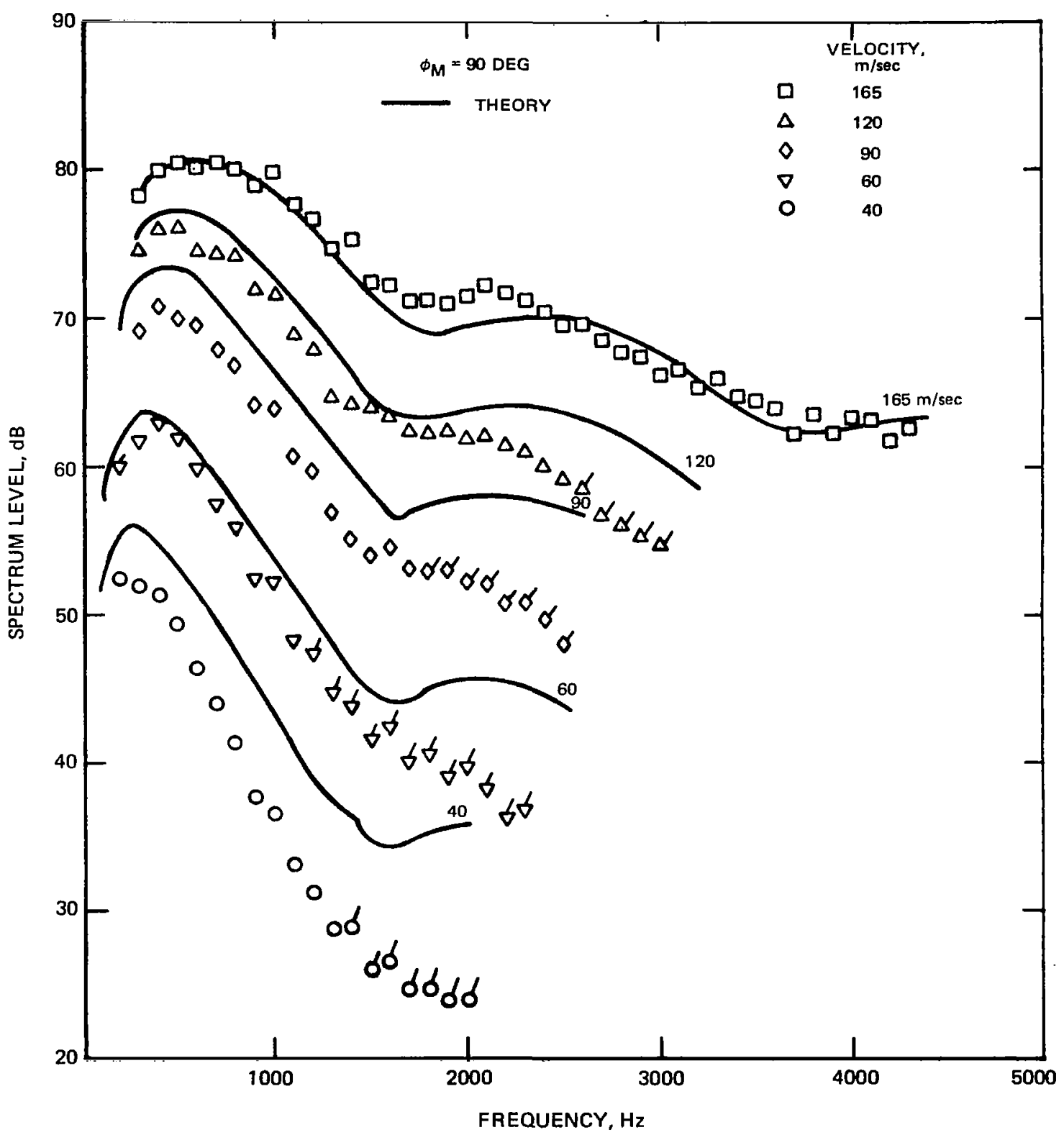

FIGURE 13.- COMPARISON OF MEASURED AND THEORETICALLY PREDICTED FAR-FIELD NOISE SPECTRA.

81 


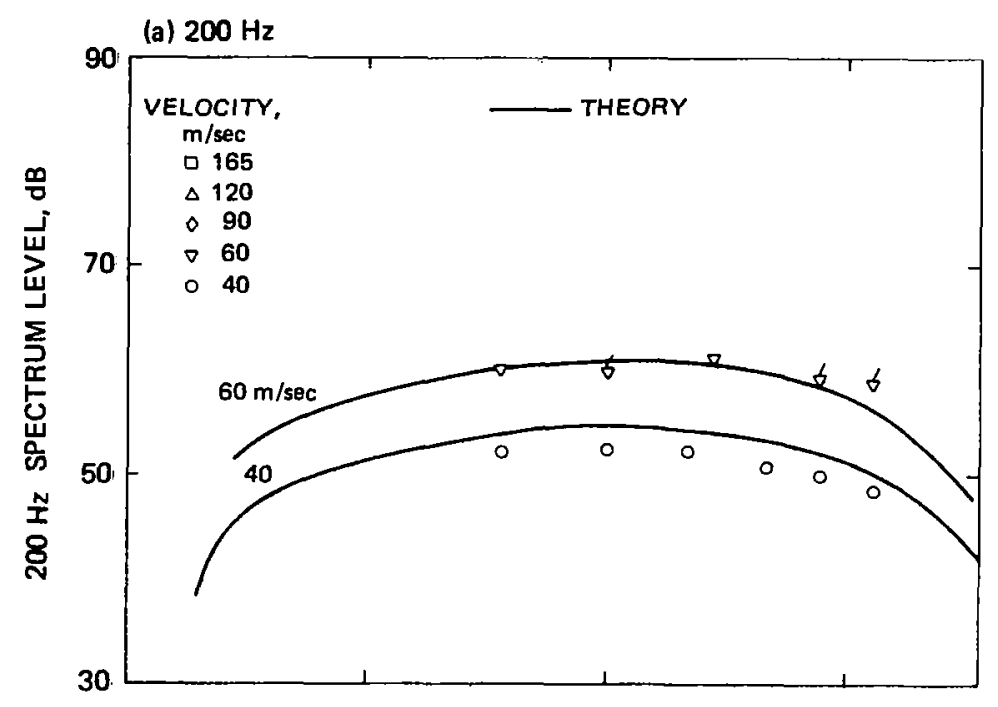

(b) $400 \mathrm{~Hz}$

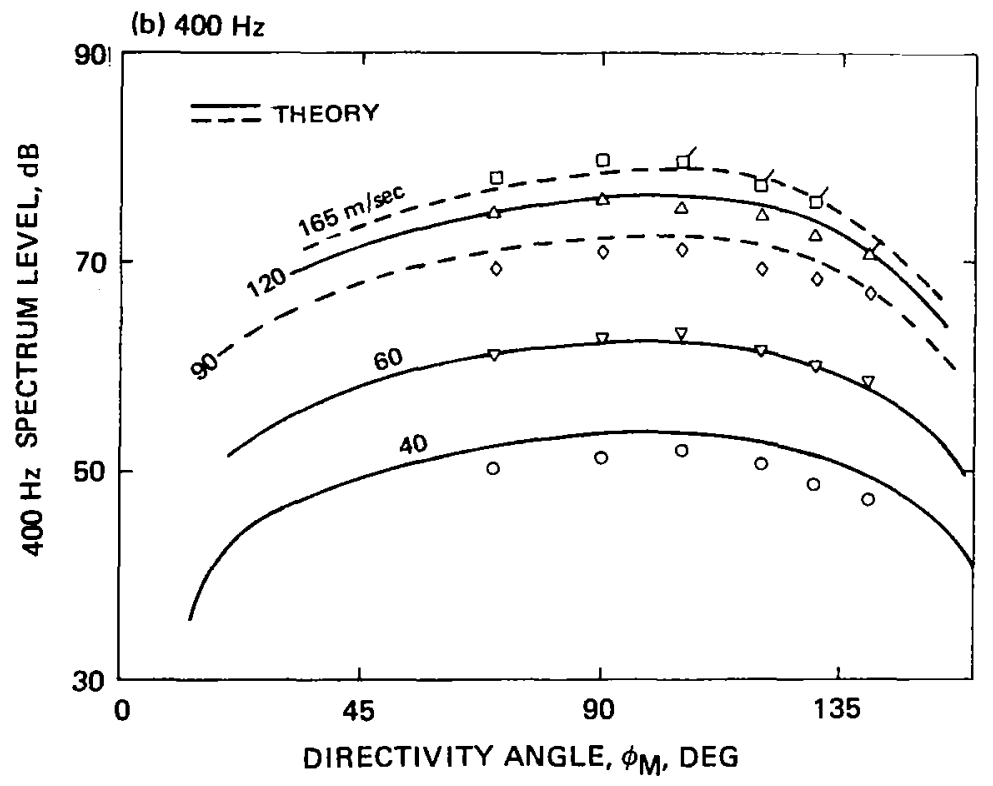

FIGURE 14.- COMPARISON OF MEASURED AND THEORETICALLY PREDICTED FAR-FIELD NOISE DIRECTIVITY. 


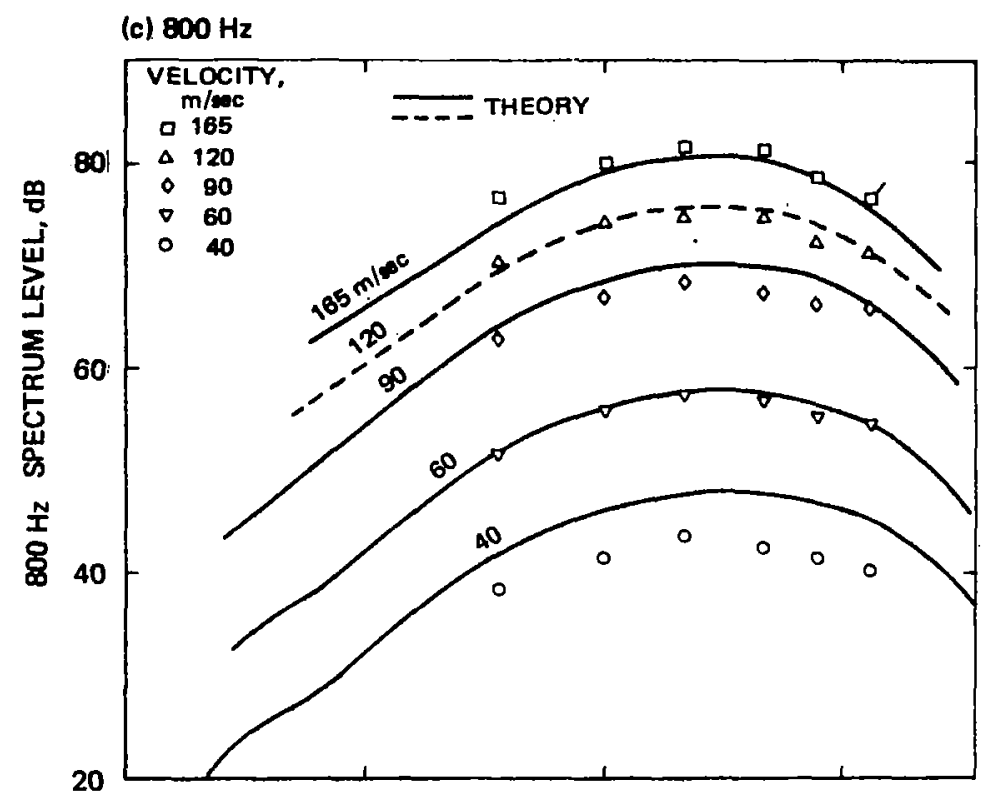

(d) $1200 \mathrm{~Hz}$

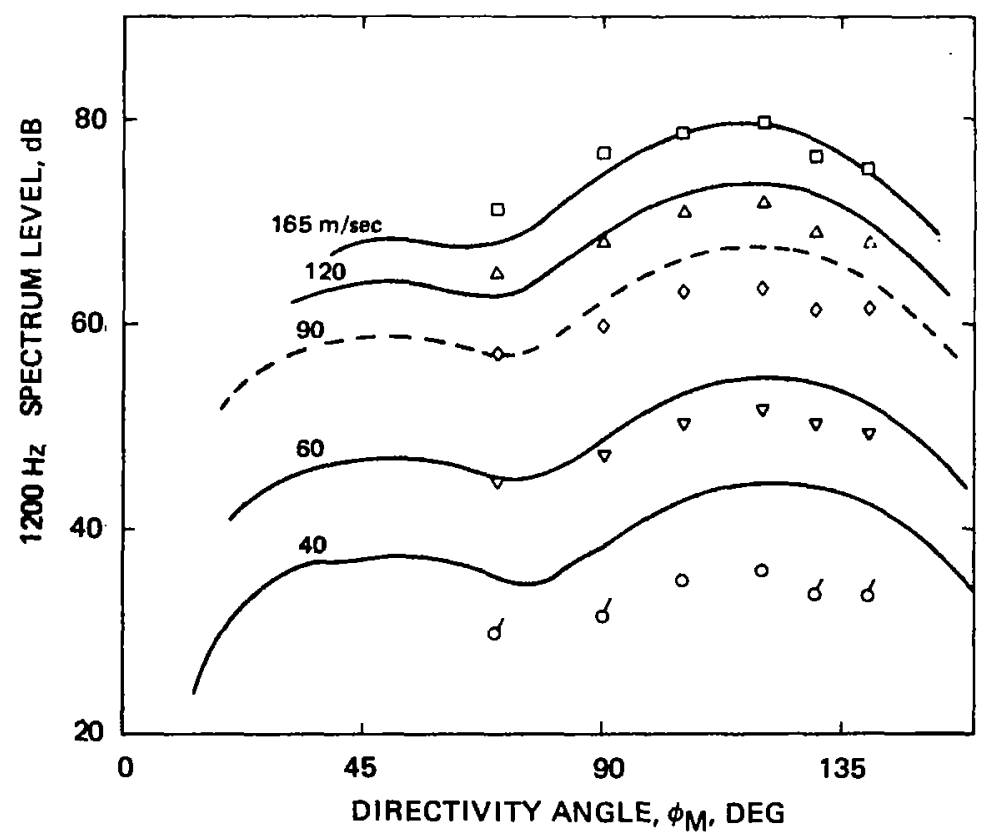

FIGURE14.- CONTINUED 

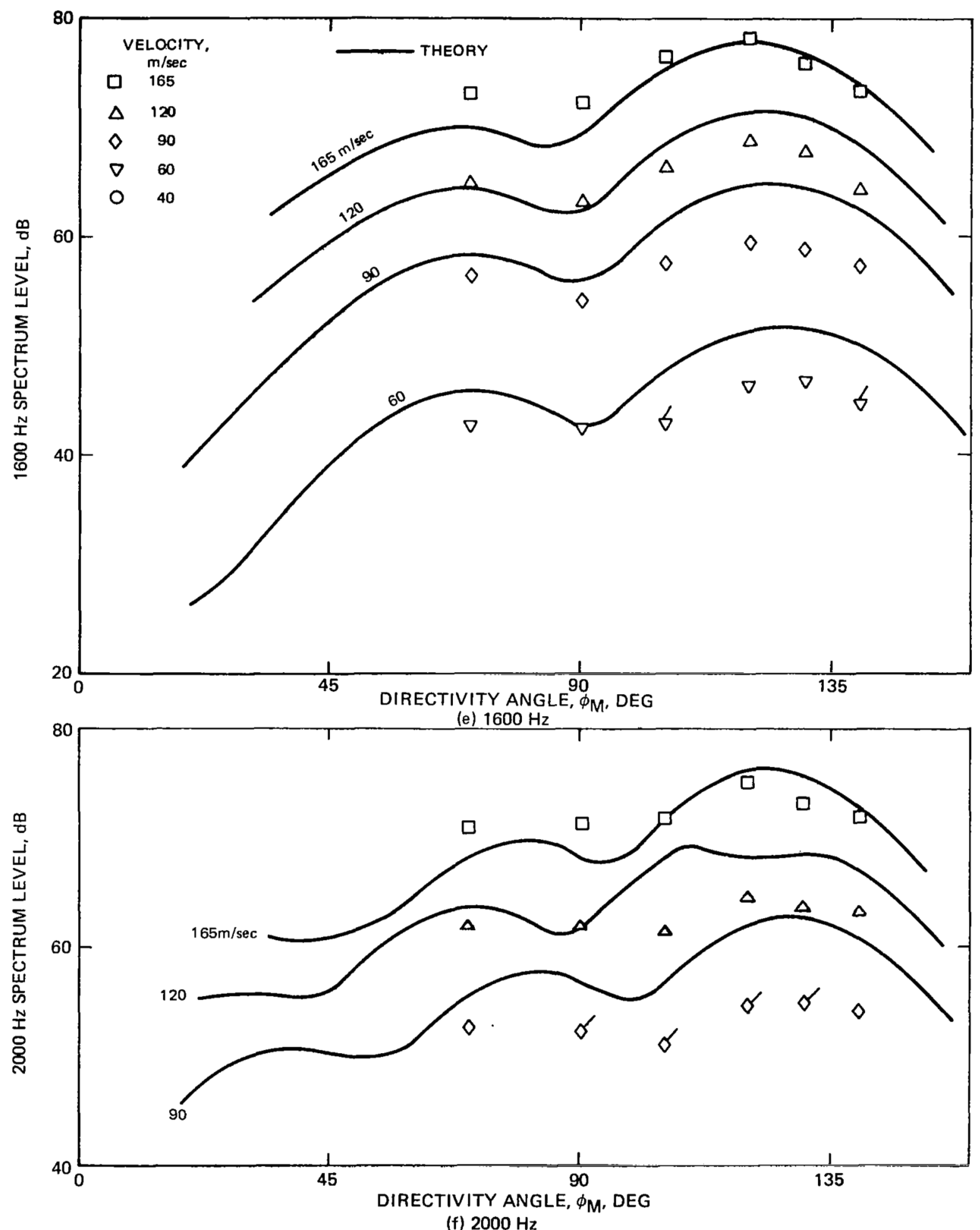

FIGURE 14.- CONTINUED 


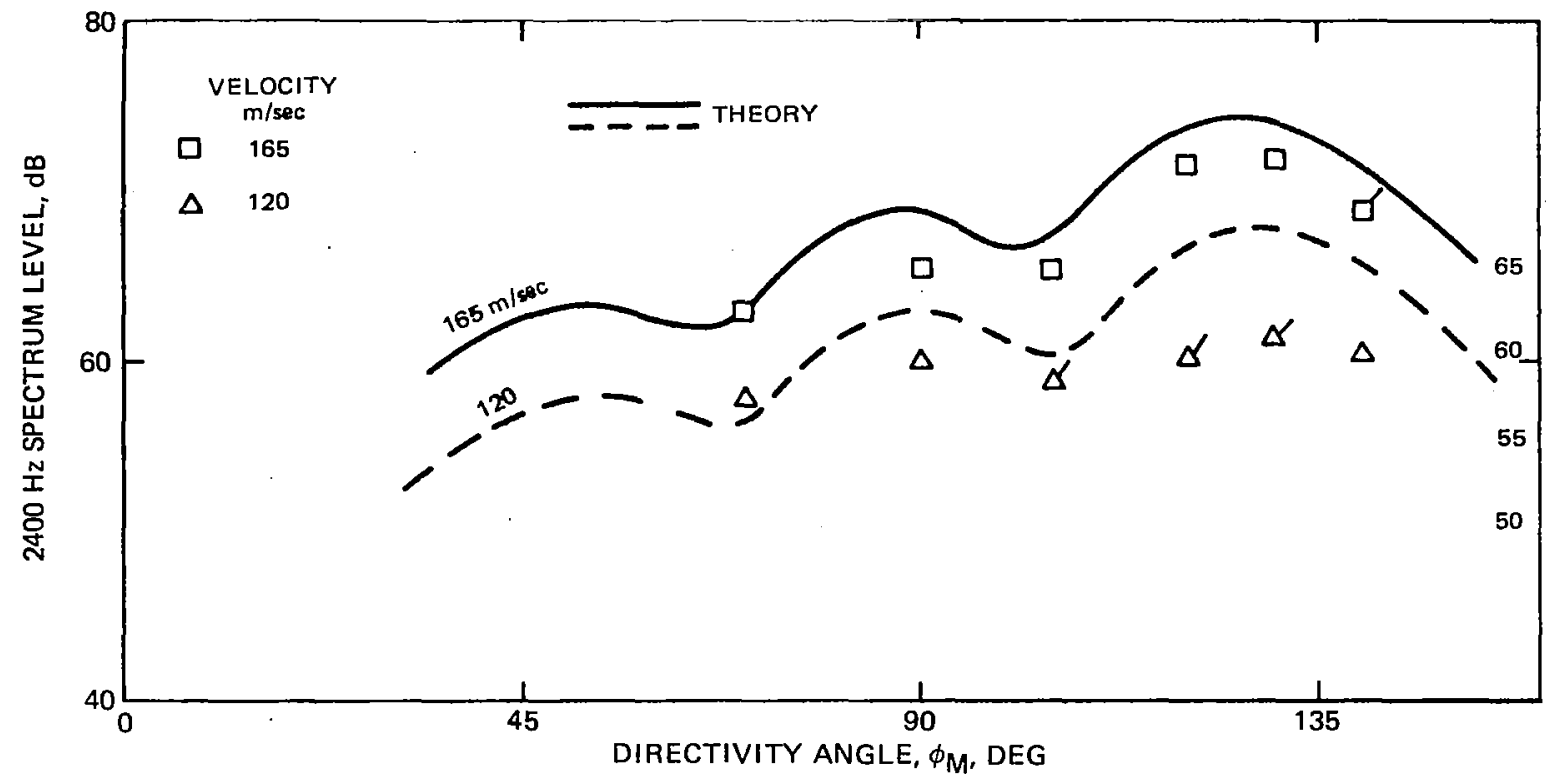

(g) $2400 \mathrm{~Hz}$

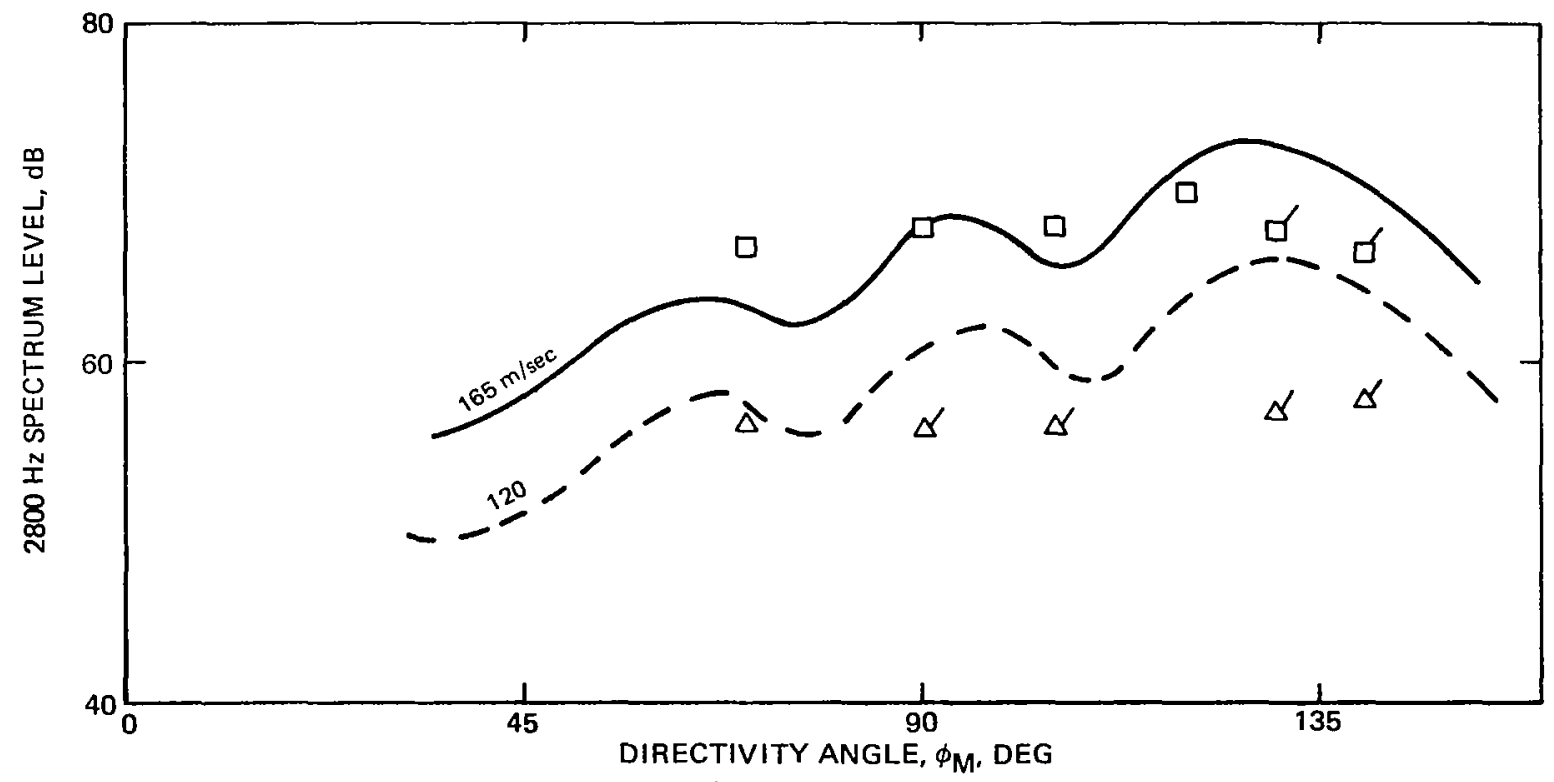

(h) $2800 \mathrm{~Hz}$

FIGURE14.- CONTINUED 

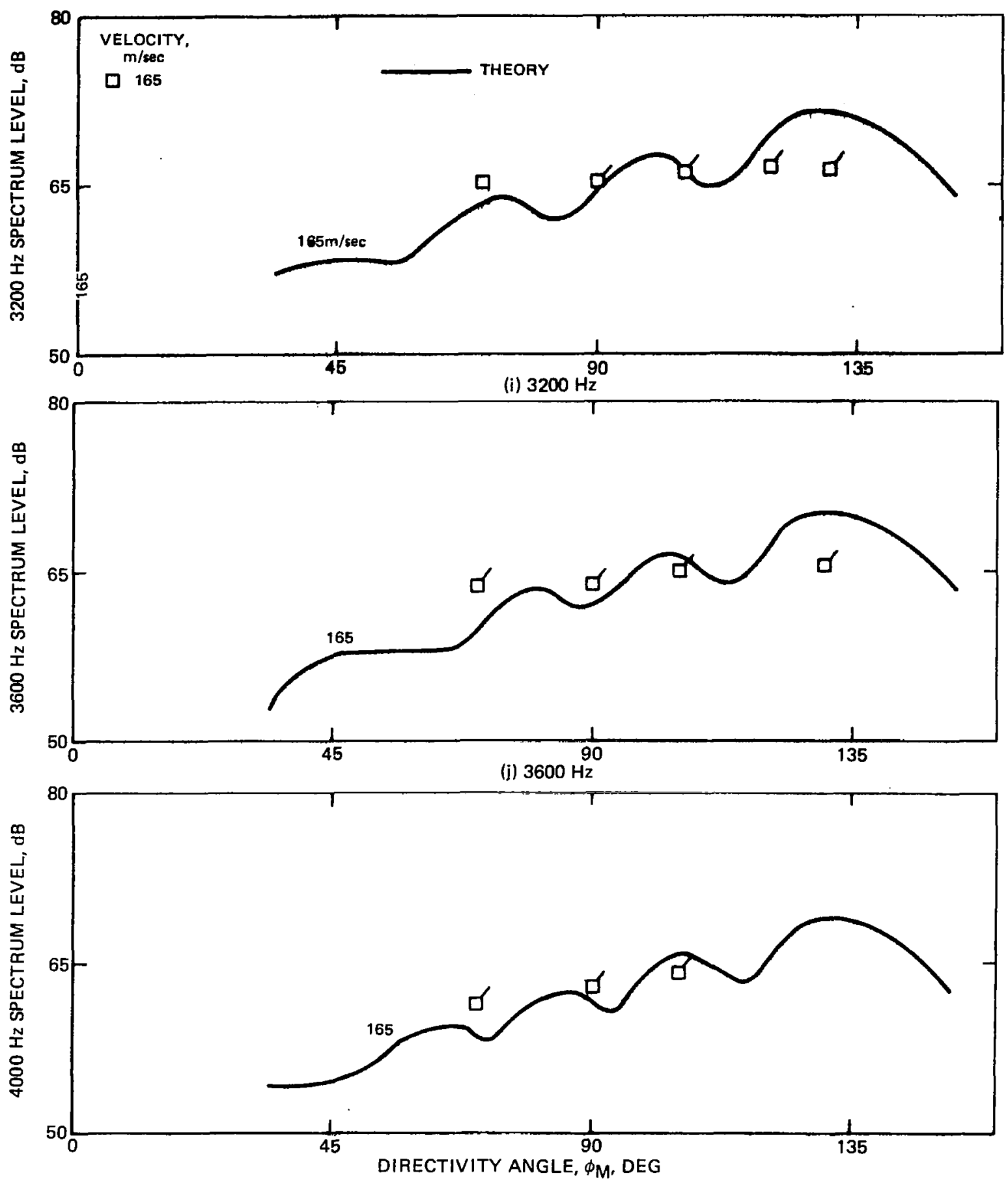

(k) $4000 \mathrm{~Hz}$

FIGURE 14.- CONCLUDED 


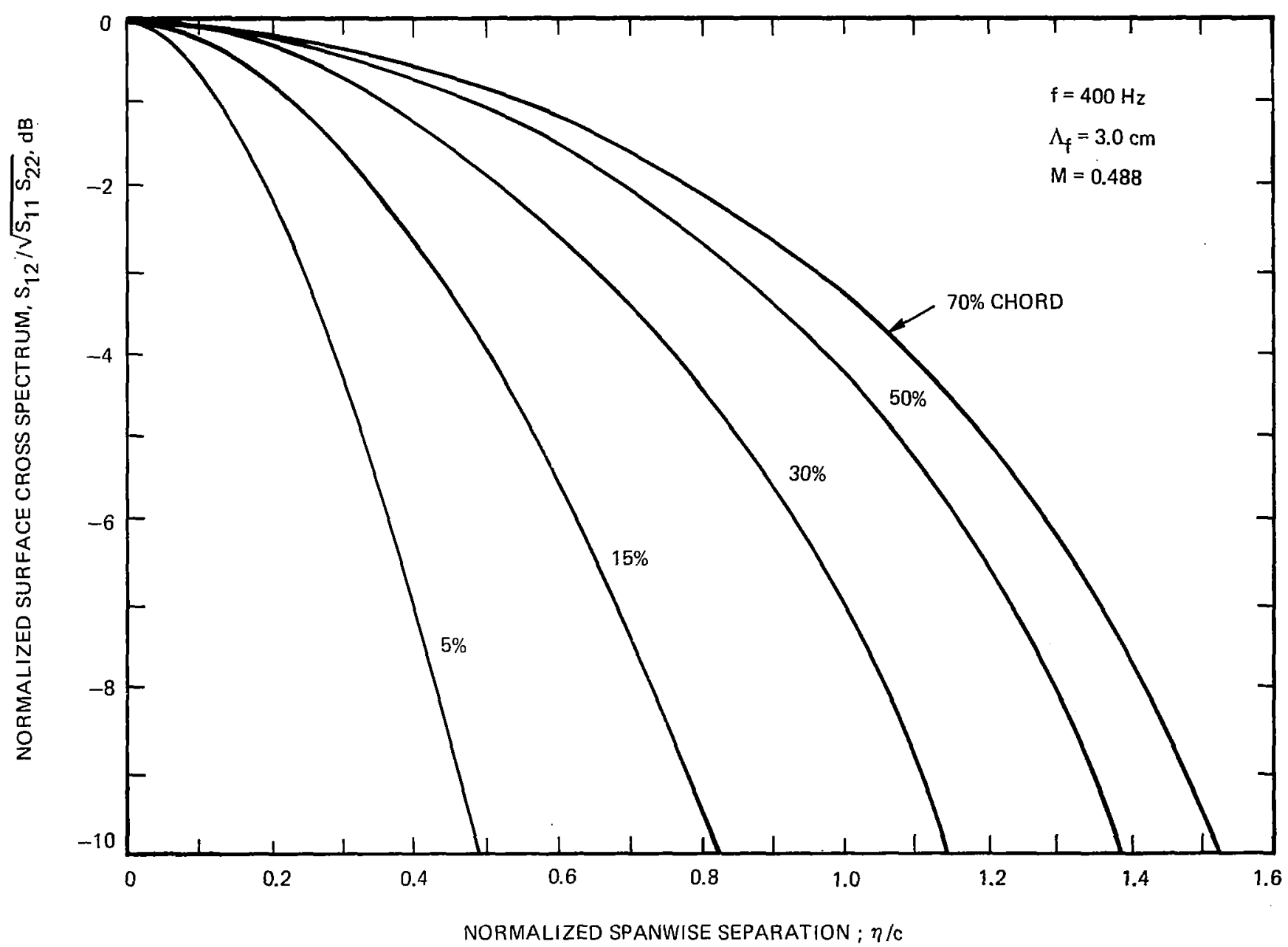

FIGURE 15. - MAGNITUDE OF CALCULATED NORMALIZED SPANWISE CROSS - SPECTRUM 


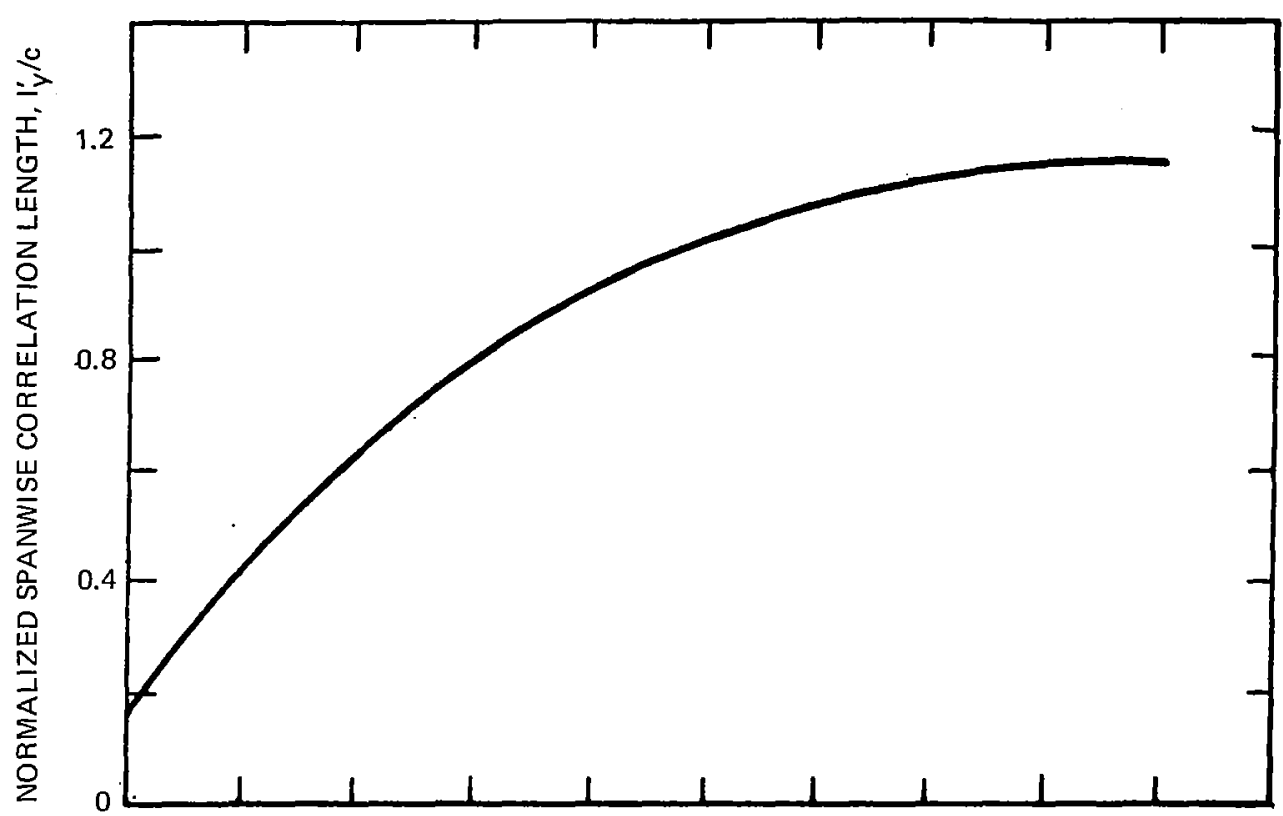

(a)

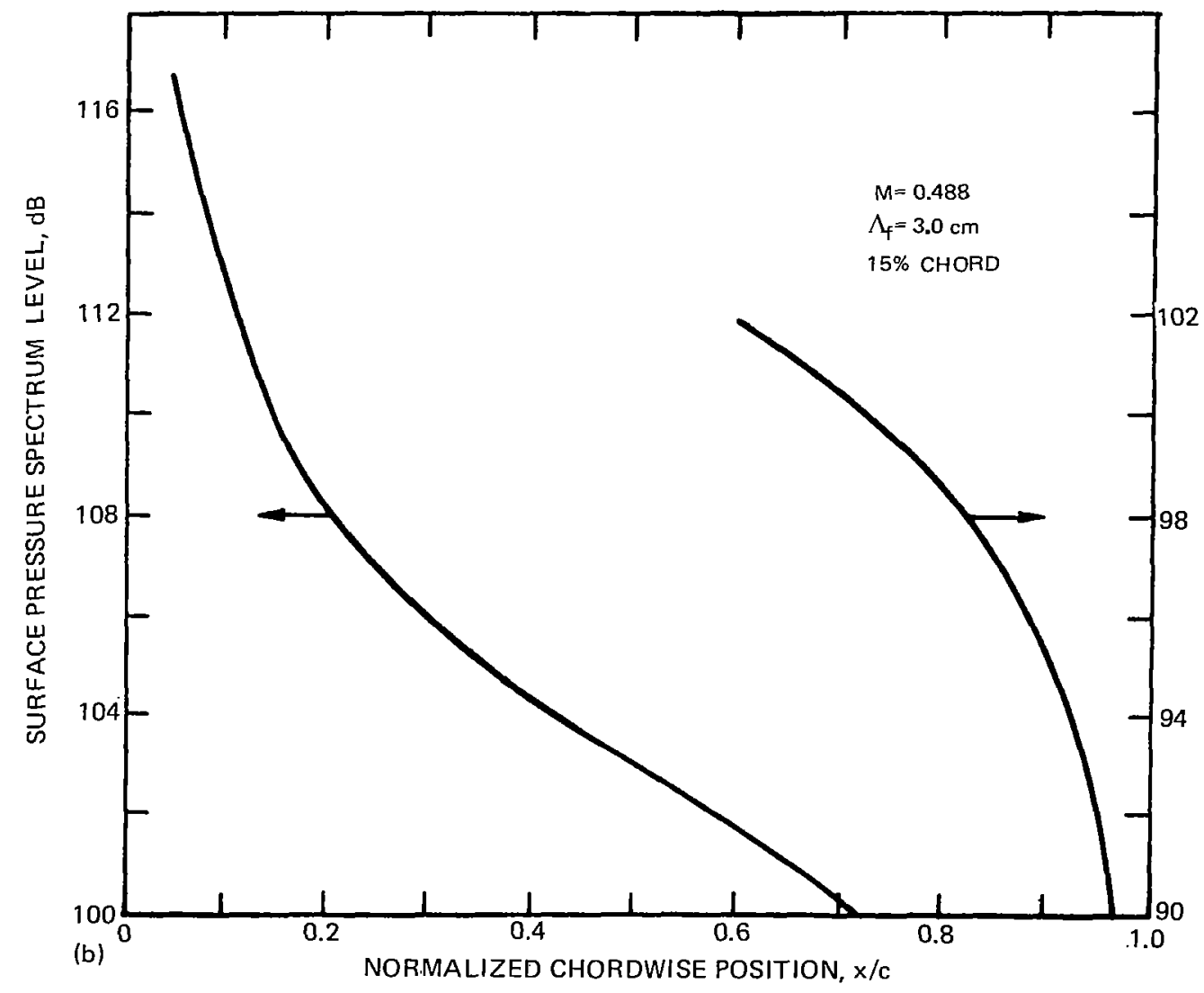

FIGURE 16. - CALCULATED SPANWISE CORRELATION LENGTH AND SPECTRUM LEVEL OF SURFACE PRESSURE 


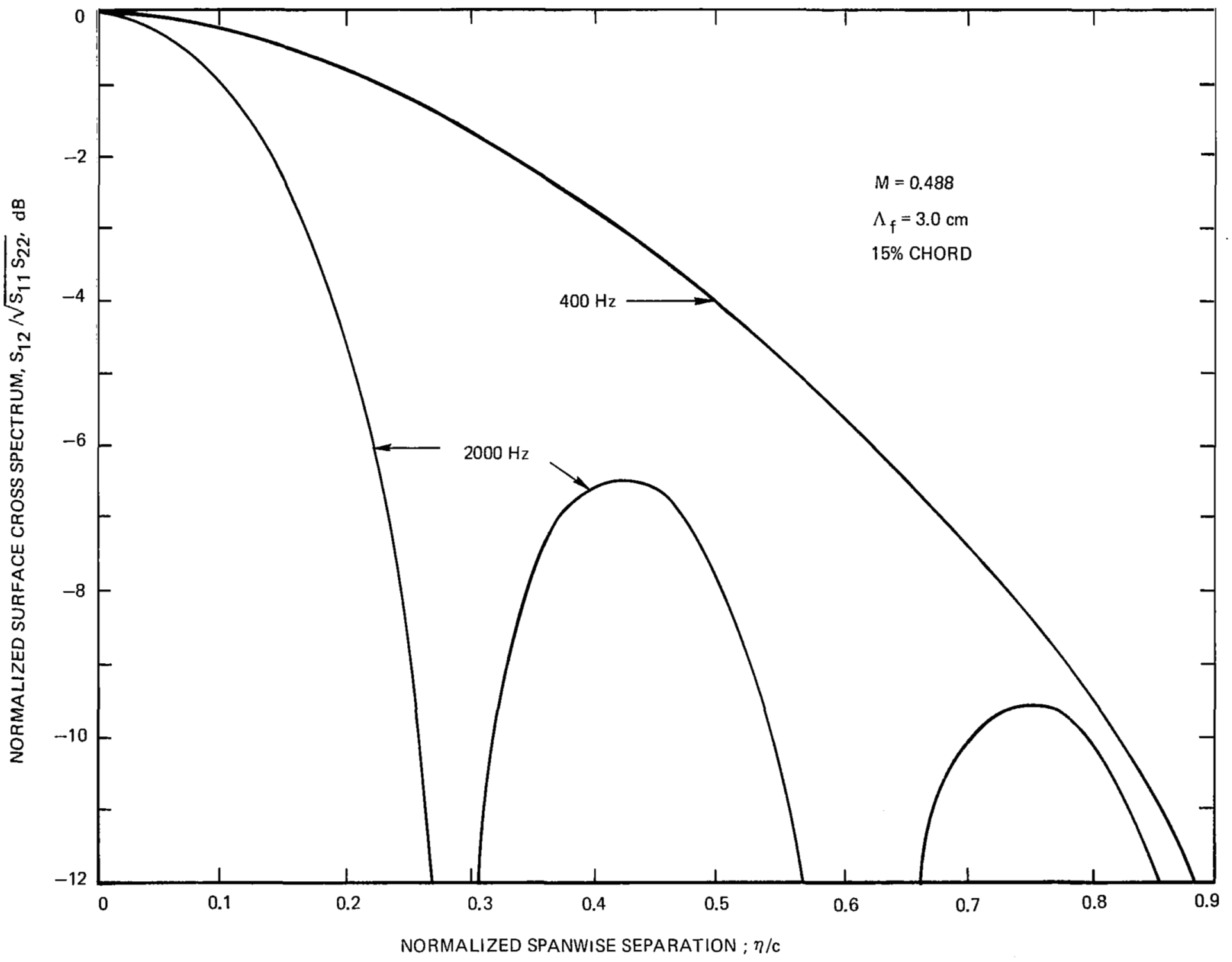

FIGURE 17. - MAGNITUDE OF CALCULATED NORMALIZED SPANWISE CROSS - SPECTRUM 


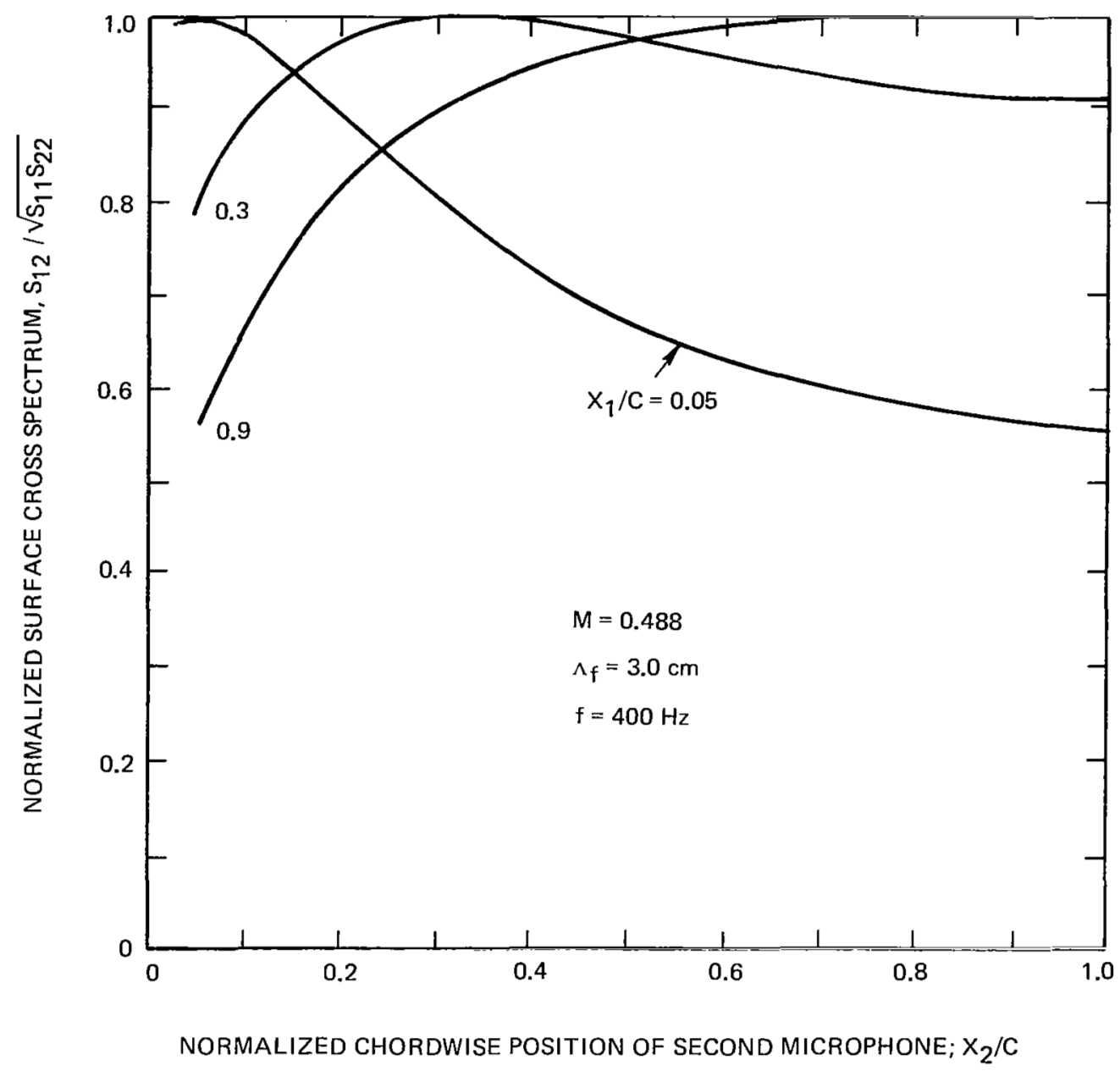

FIGURE 18. - MAGNITUDE OF CALCULATED NORMALIZED CHORDWISE CROSS- SPECTRUM 


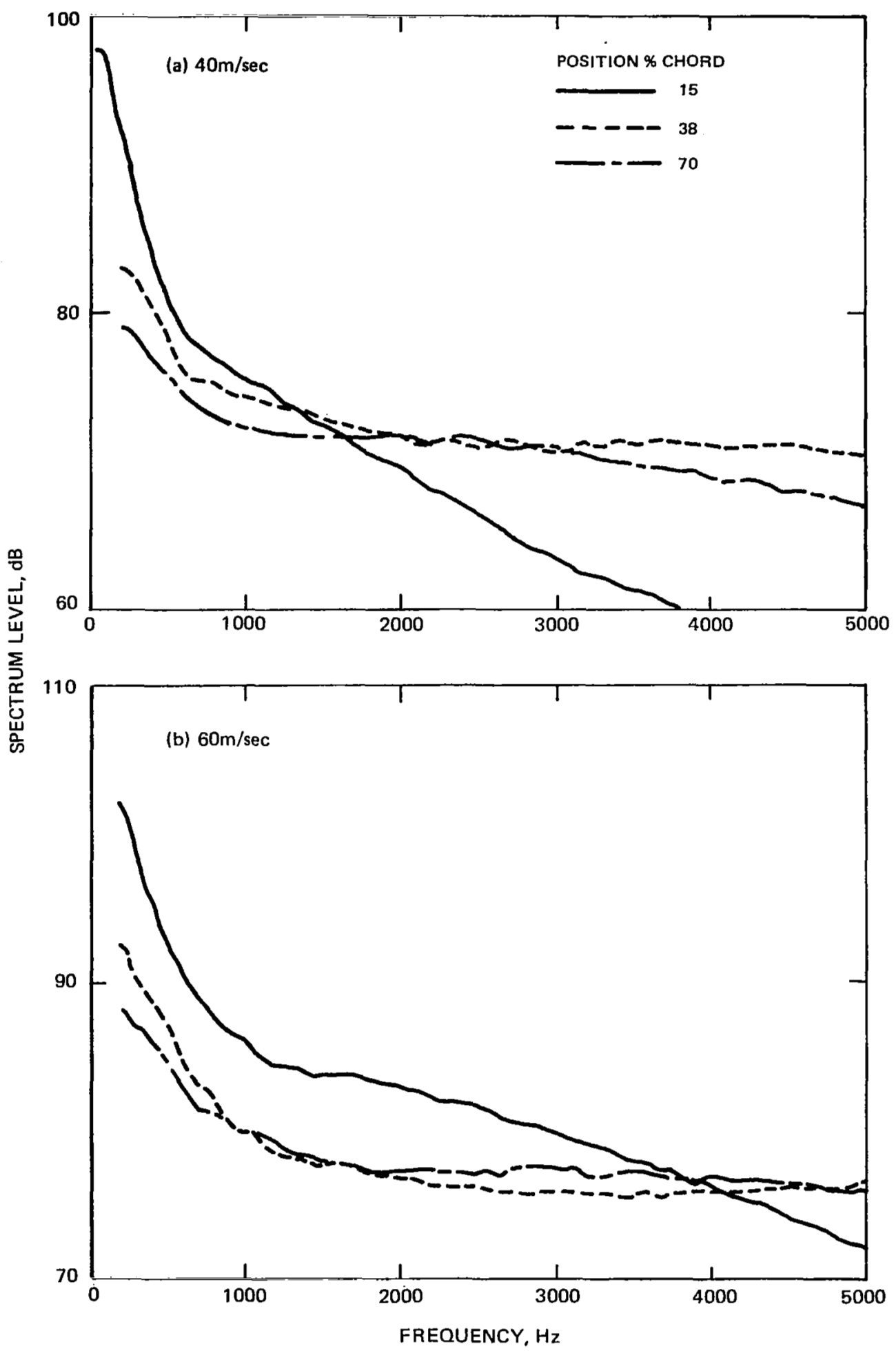

FIGURE19,-SURFACE PRESSURE SPECTRA 


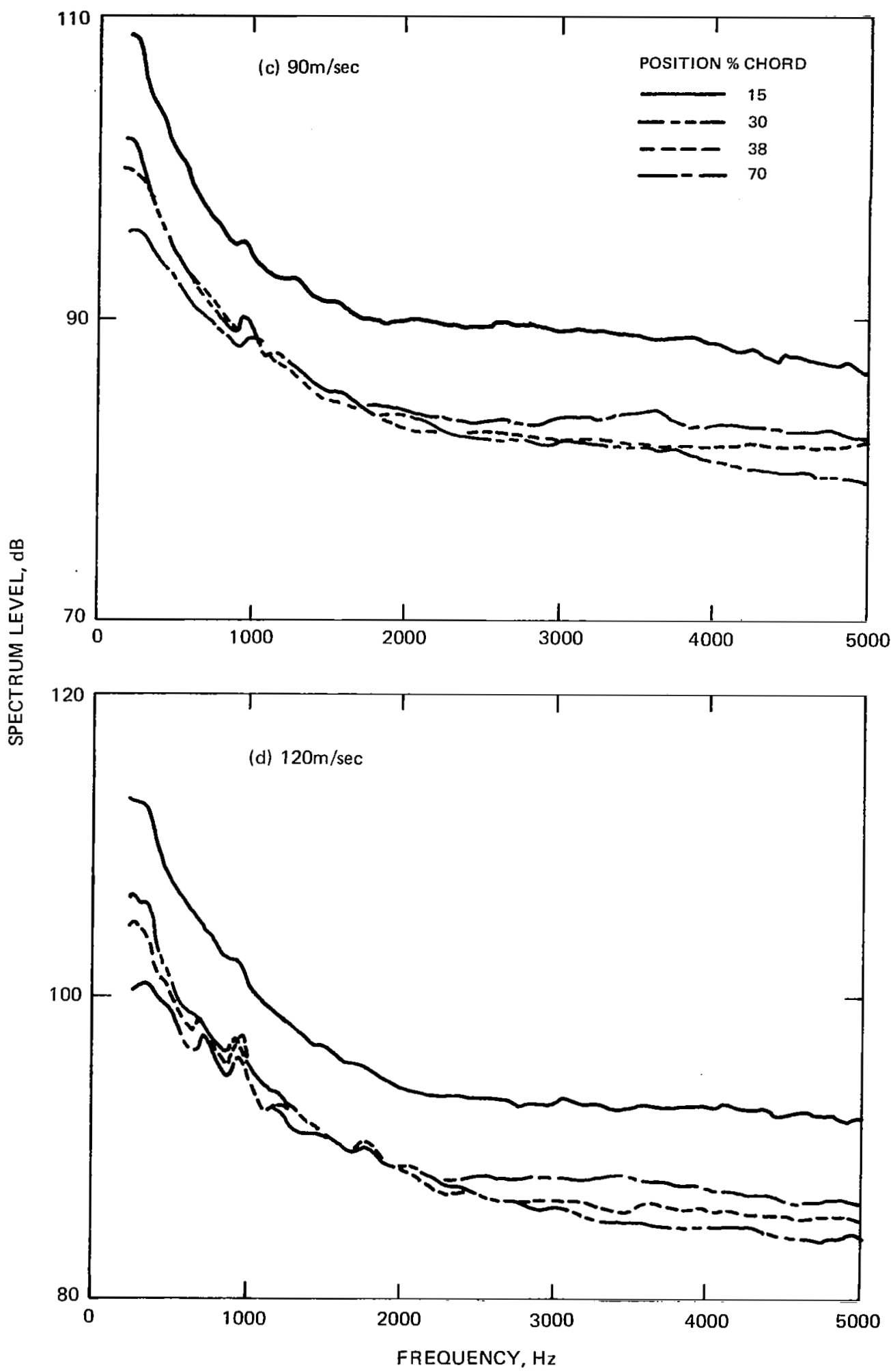

FIGURE 19.- (CONCLUDED) 

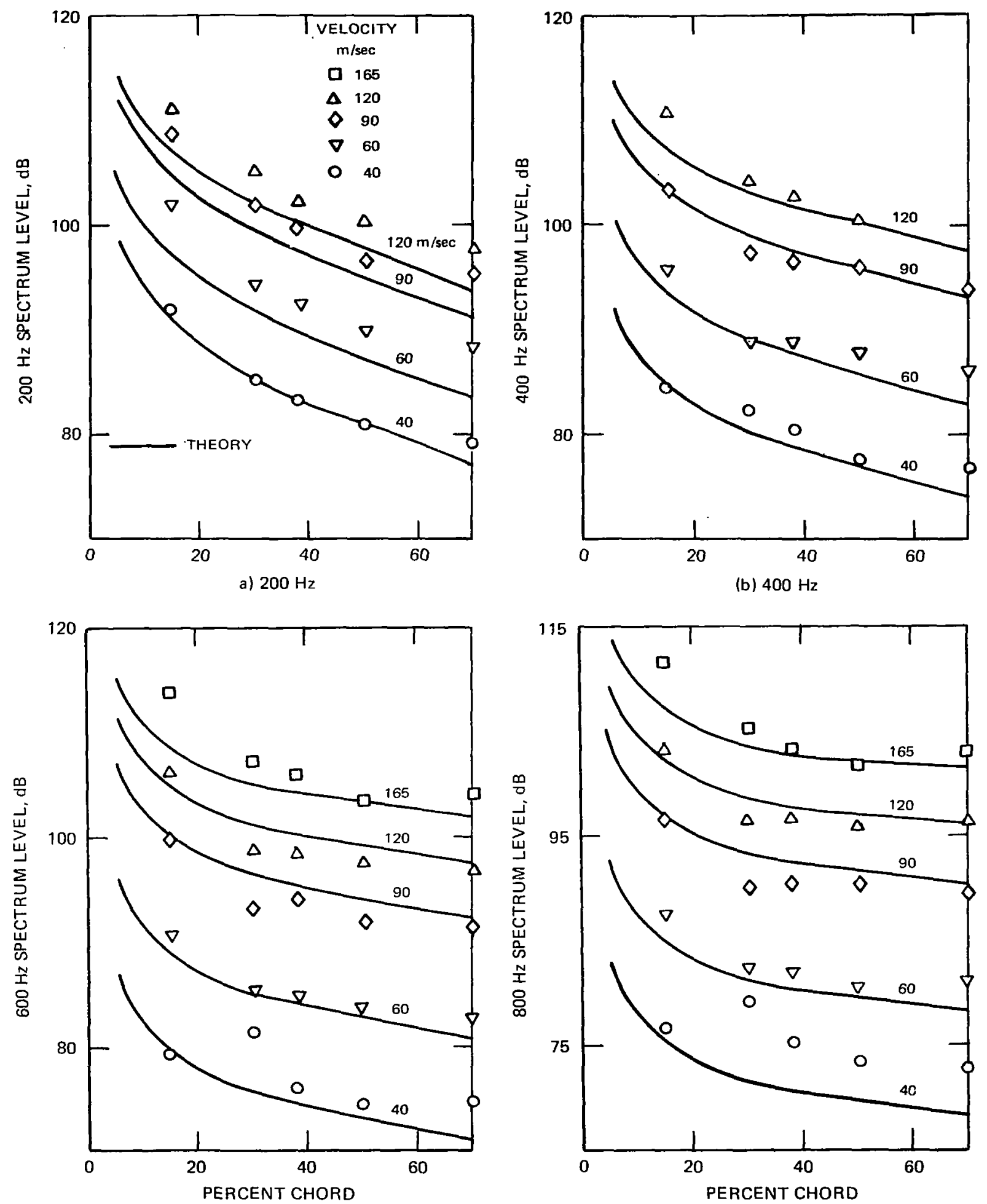

(c) $600 \mathrm{~Hz}$

(d) $800 \mathrm{~Hz}$.

FIGURE 20. - COMPARISON OF MEASURED AND THEORETICALLY PREDICTED SURFACE PRESSURE DISTRIBUTIONS 


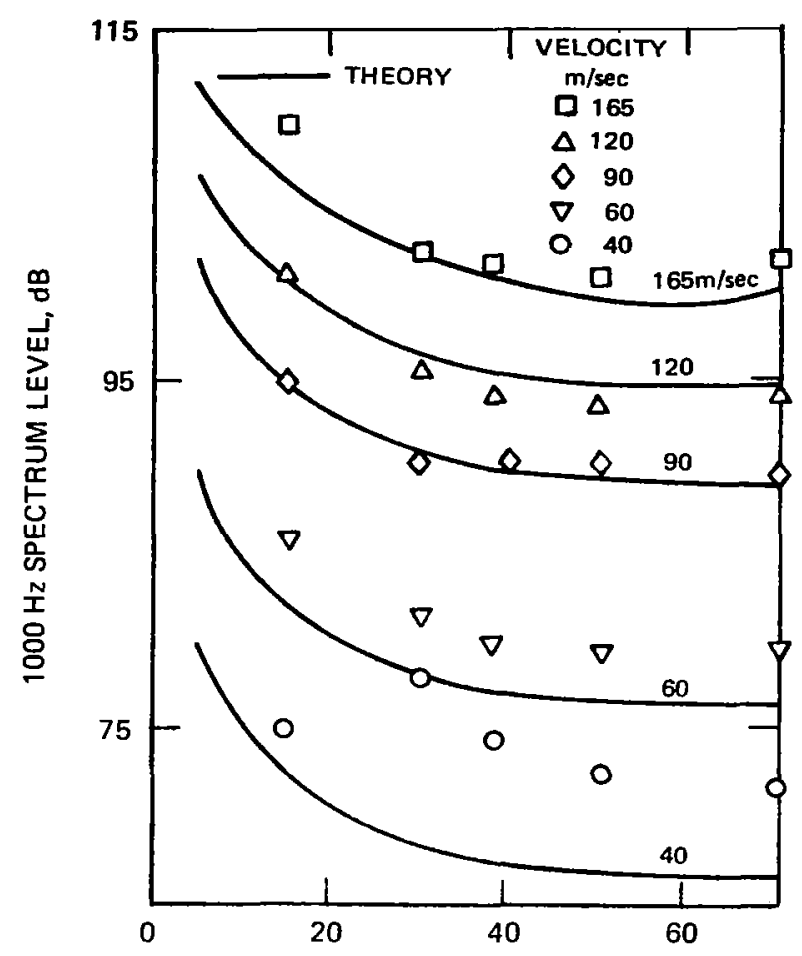

(e) $1000 \mathrm{~Hz}$

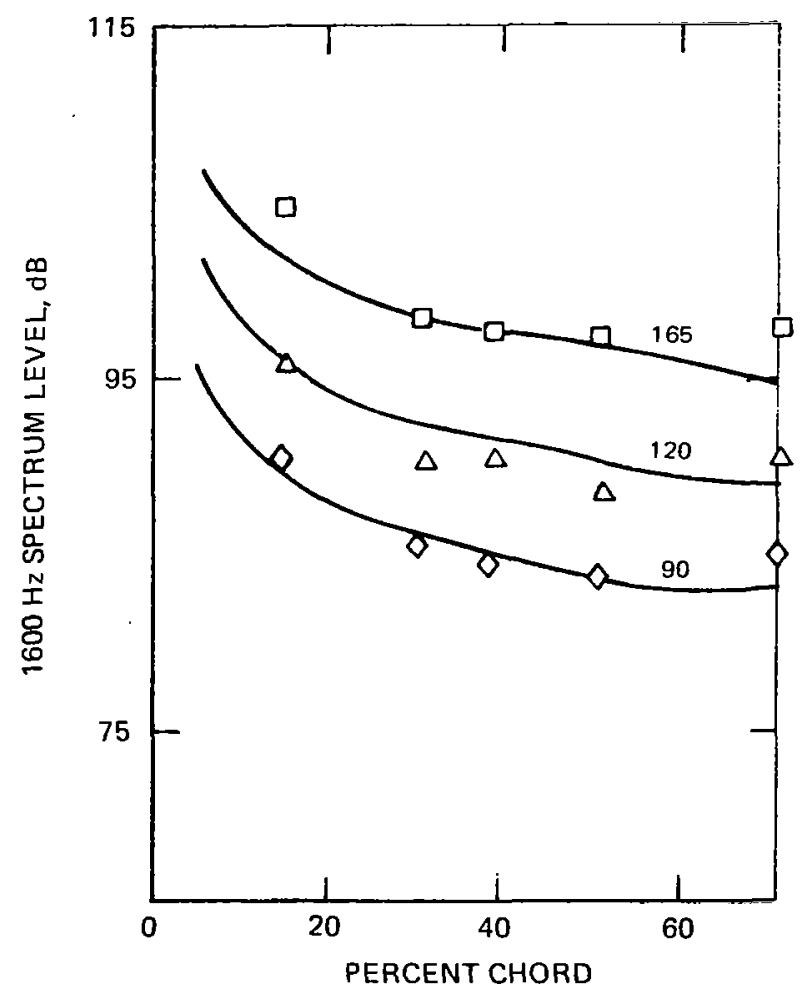

(g) $1600 \mathrm{~Hz}$

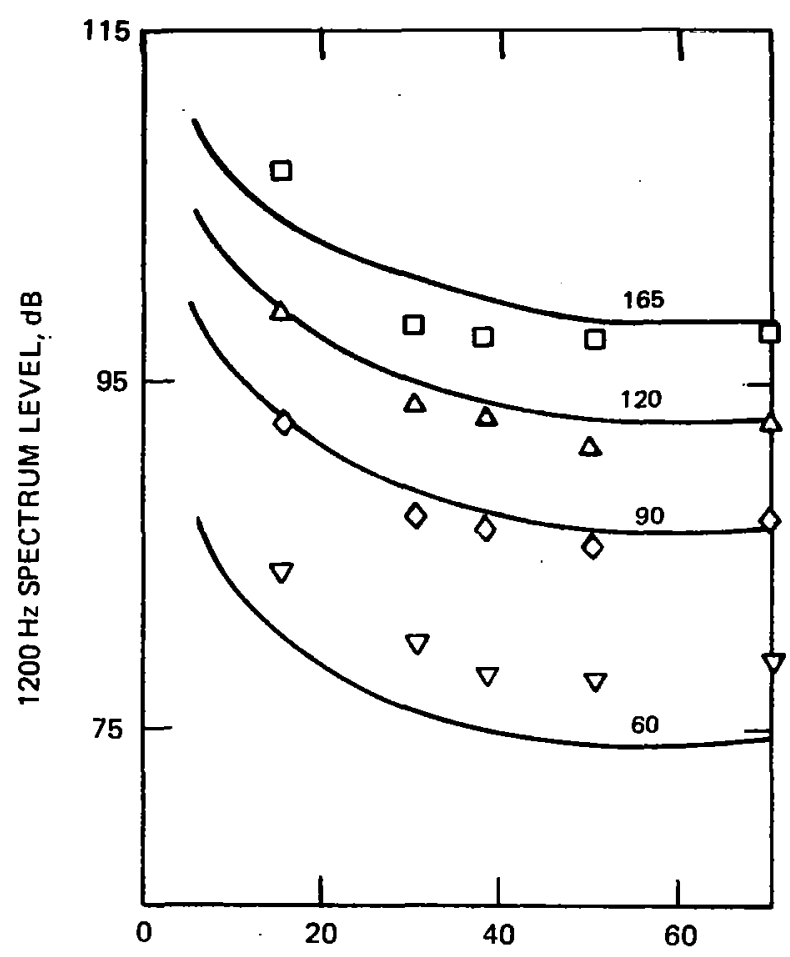

(f) $1200 \mathrm{~Hz}$

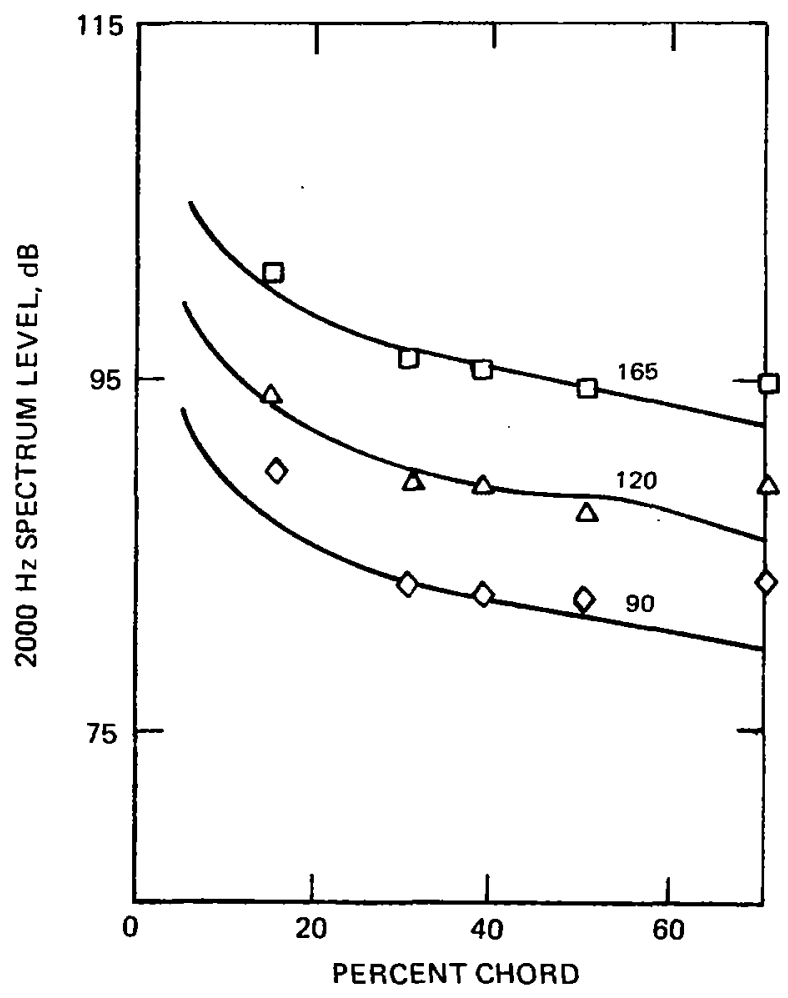

(h) 2000

FIGURE 20. - CONTINUED 

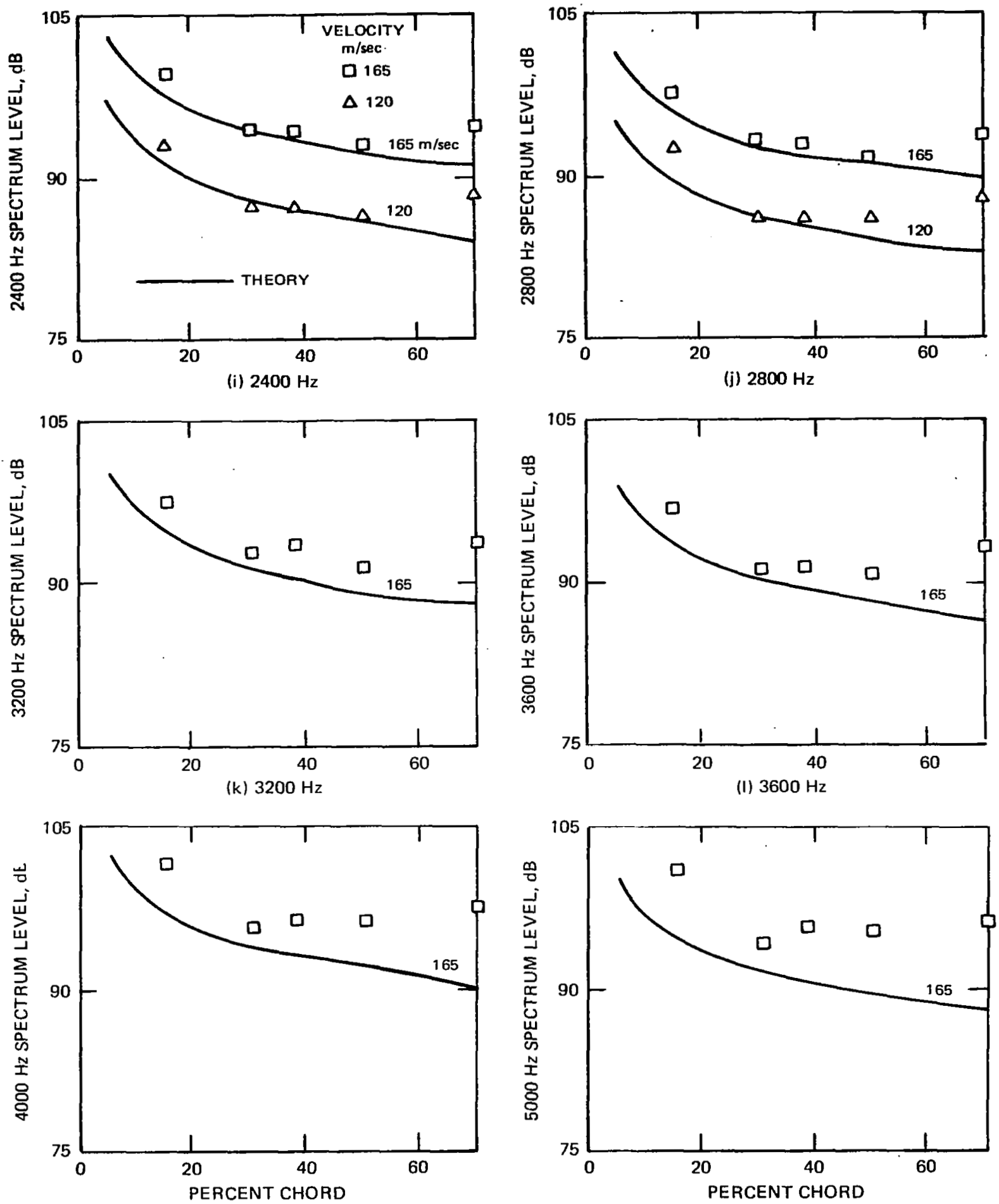

(n) $5000 \mathrm{~Hz}$

FIGURE 20. - CONCLUDED 


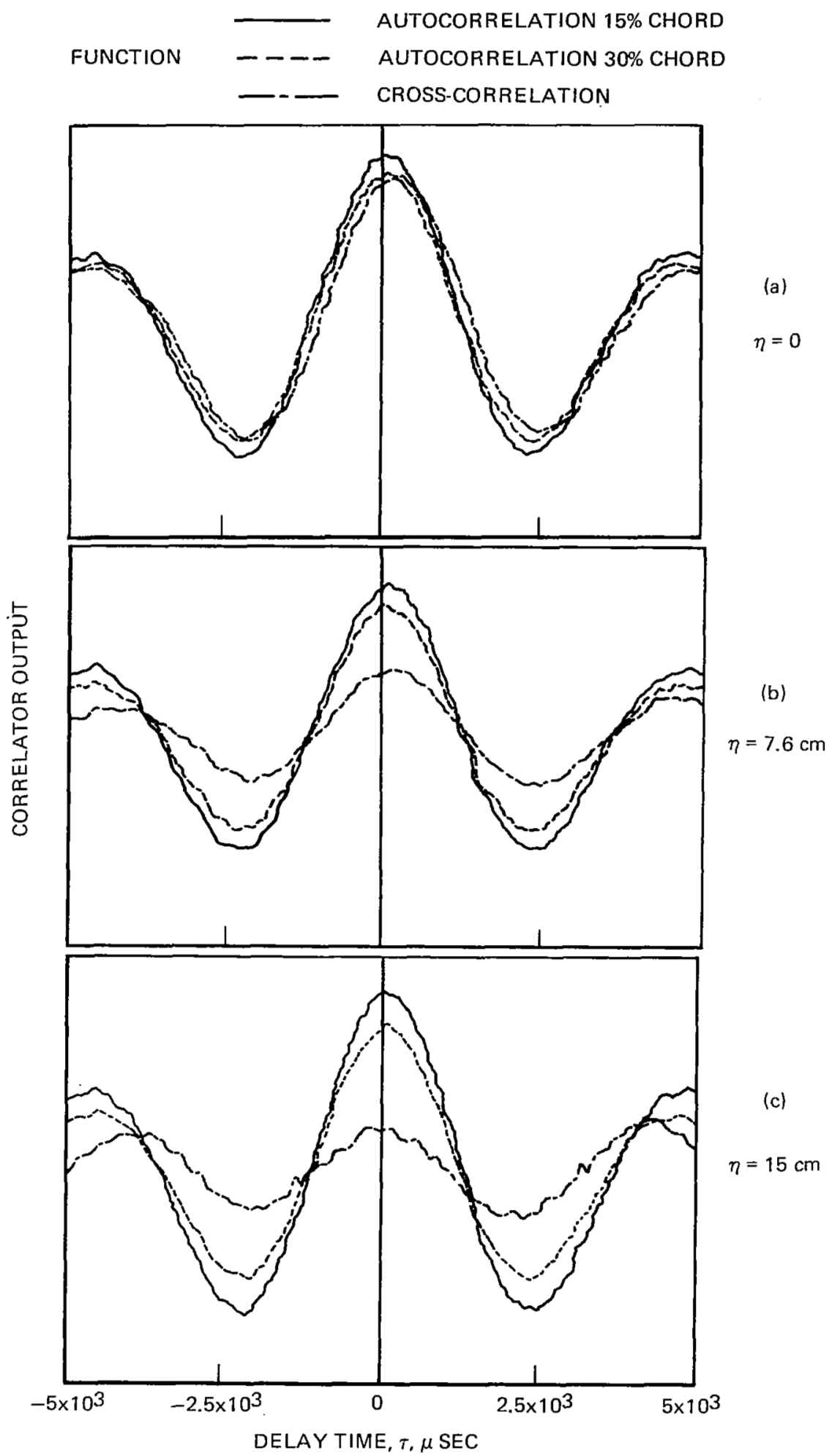

FIGURE 21. - VARIATION OF $200 \mathrm{~Hz}$ PREFILTERED SURFACE PRESSURE CORRELATIONS WITH SPANWISE SEPARATION 

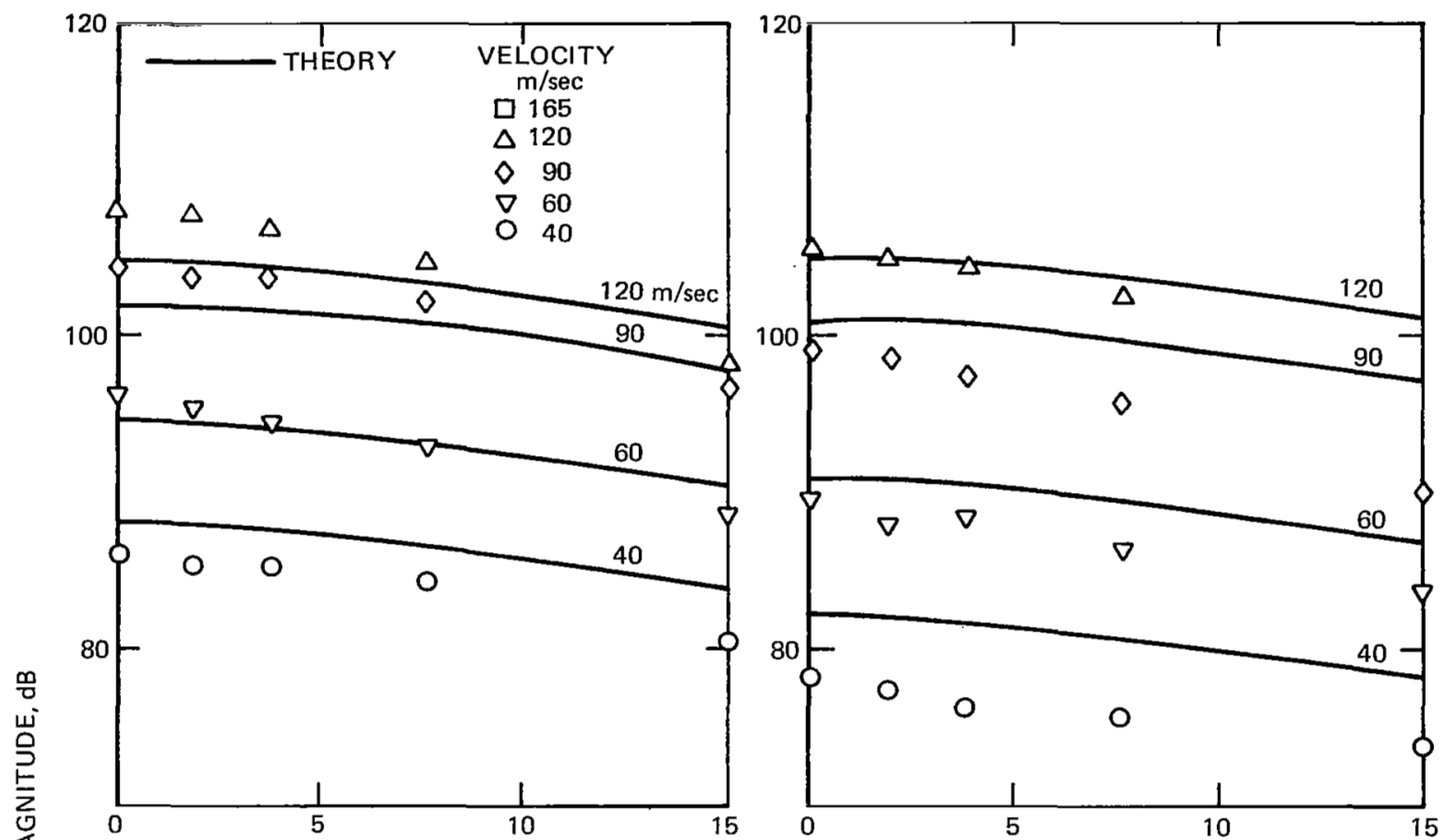

(a) $200 \mathrm{~Hz}$

(b) $400 \mathrm{~Hz}$

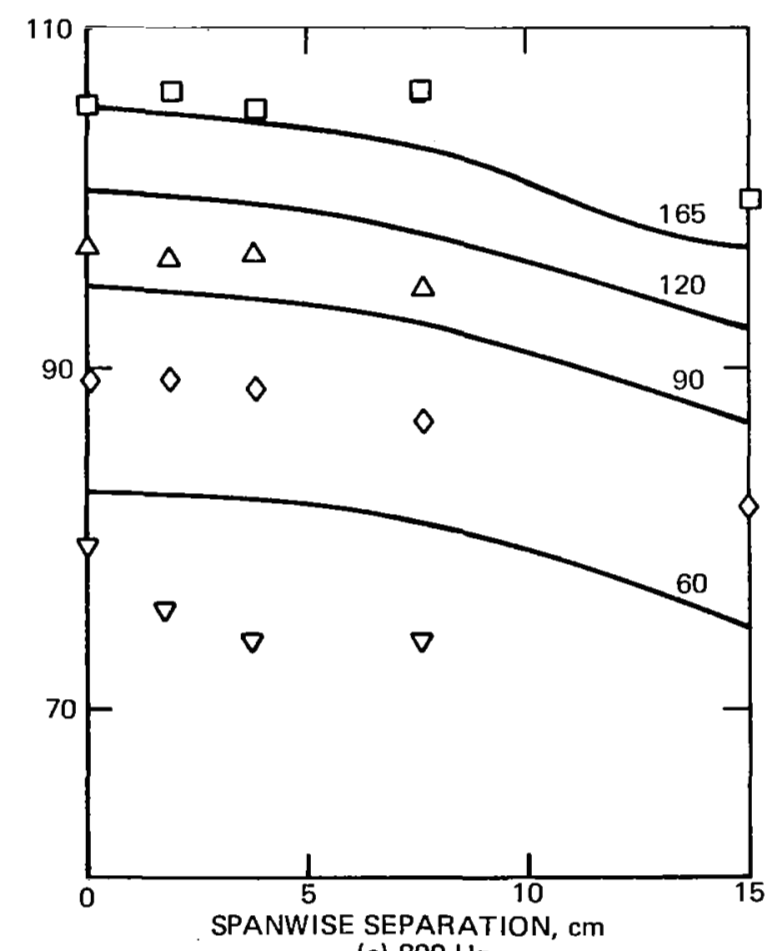

(c) $800 \mathrm{~Hz}$

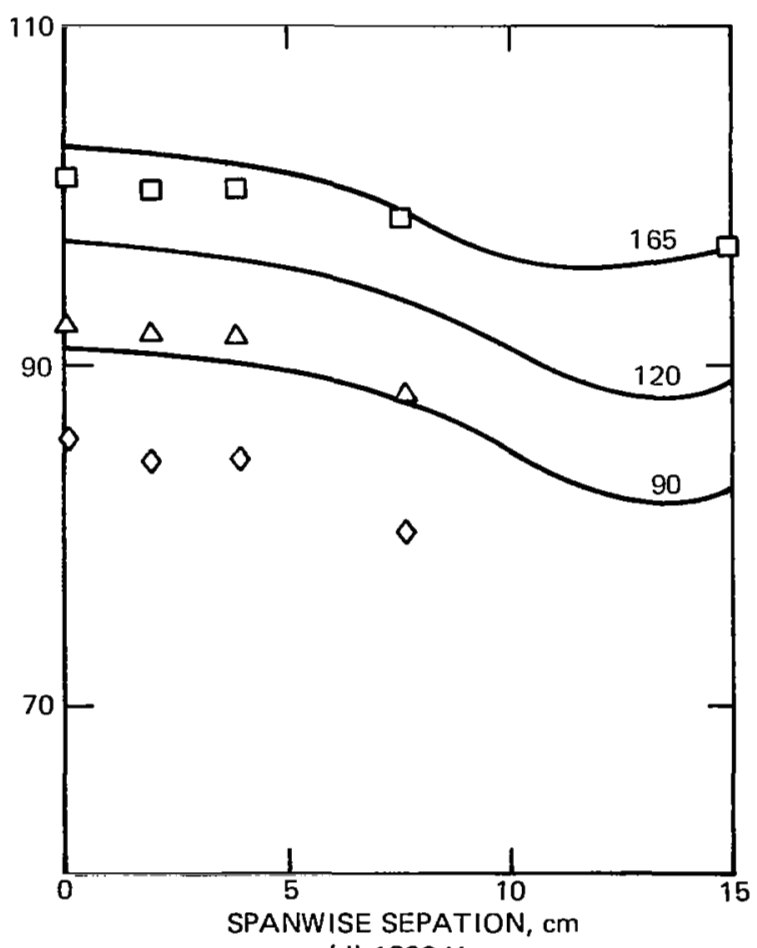

(d) $1200 \mathrm{~Hz}$

FIGURE 22.- COMPARISON OF MEASURED AND THEORETICALLY PREDICTED SURFACE PRESSURE SPANWISE CROSS-SPECTRA 


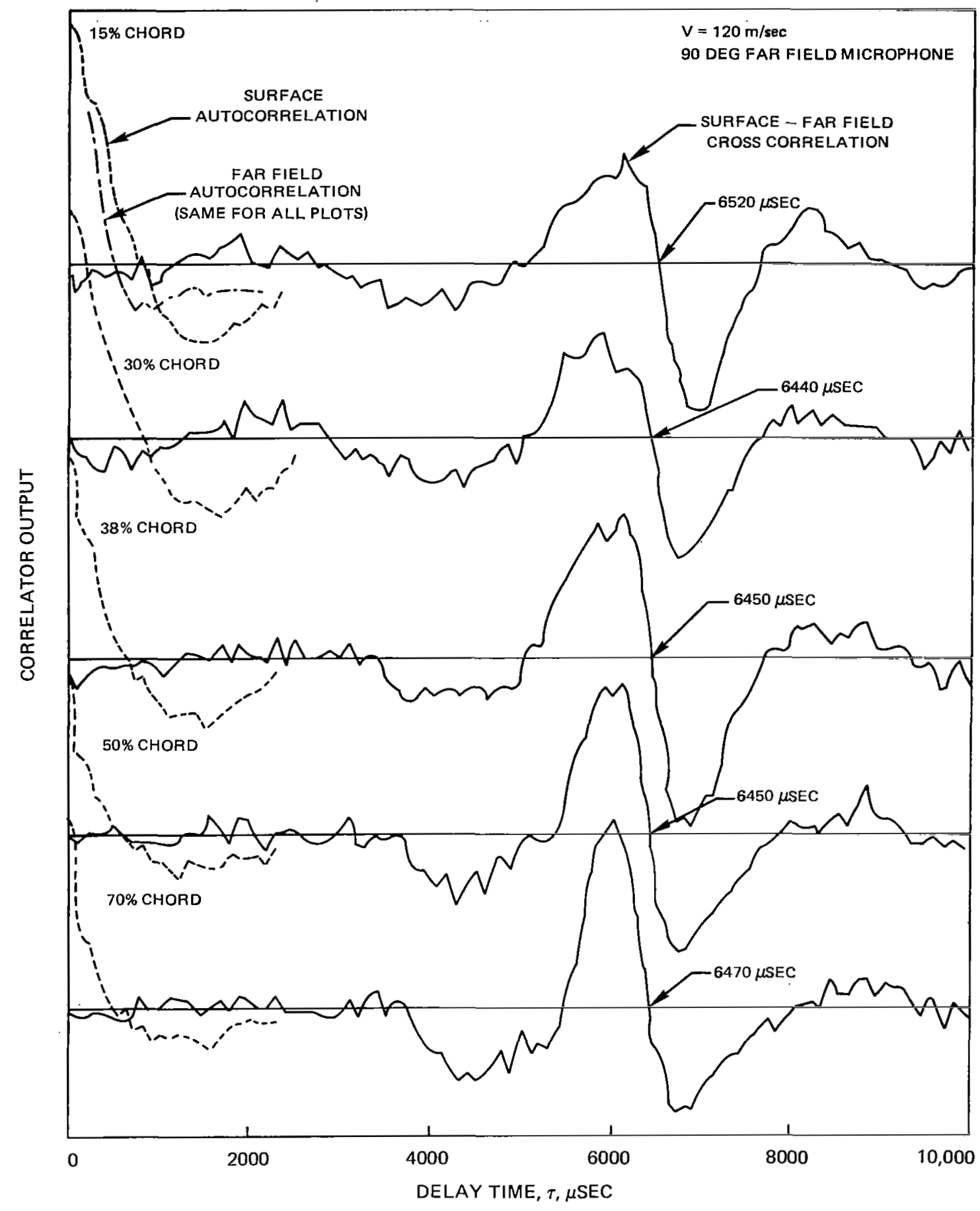

FIGURE 23. - SURFACE PRESSURE AND FAR-FIELD CORRELATIONS 

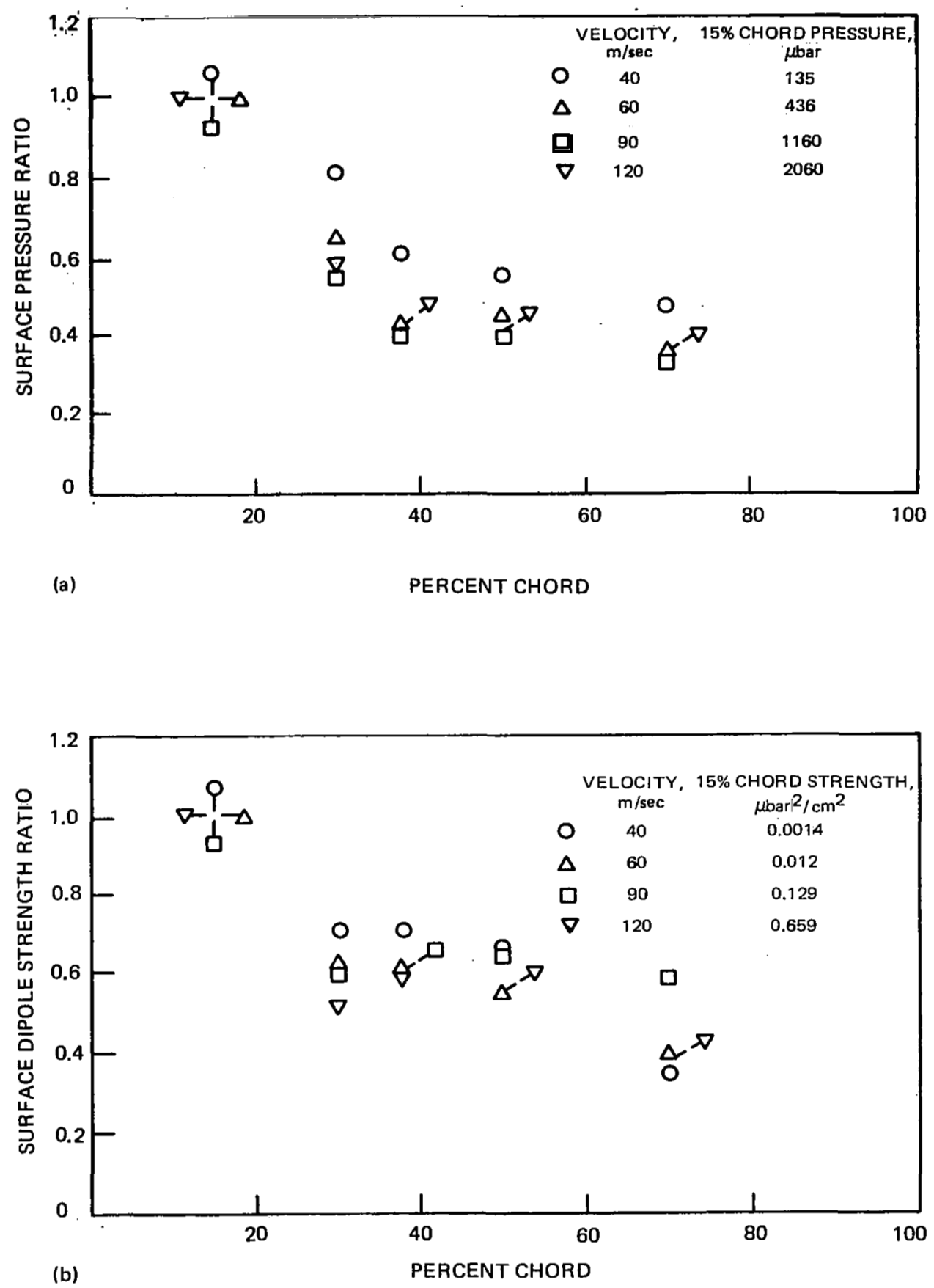

FIGURE 24. - CHORDWISE DISTRIBUTION OF SURFACE PRESSURES AND DIPOLE STRENGTHS 


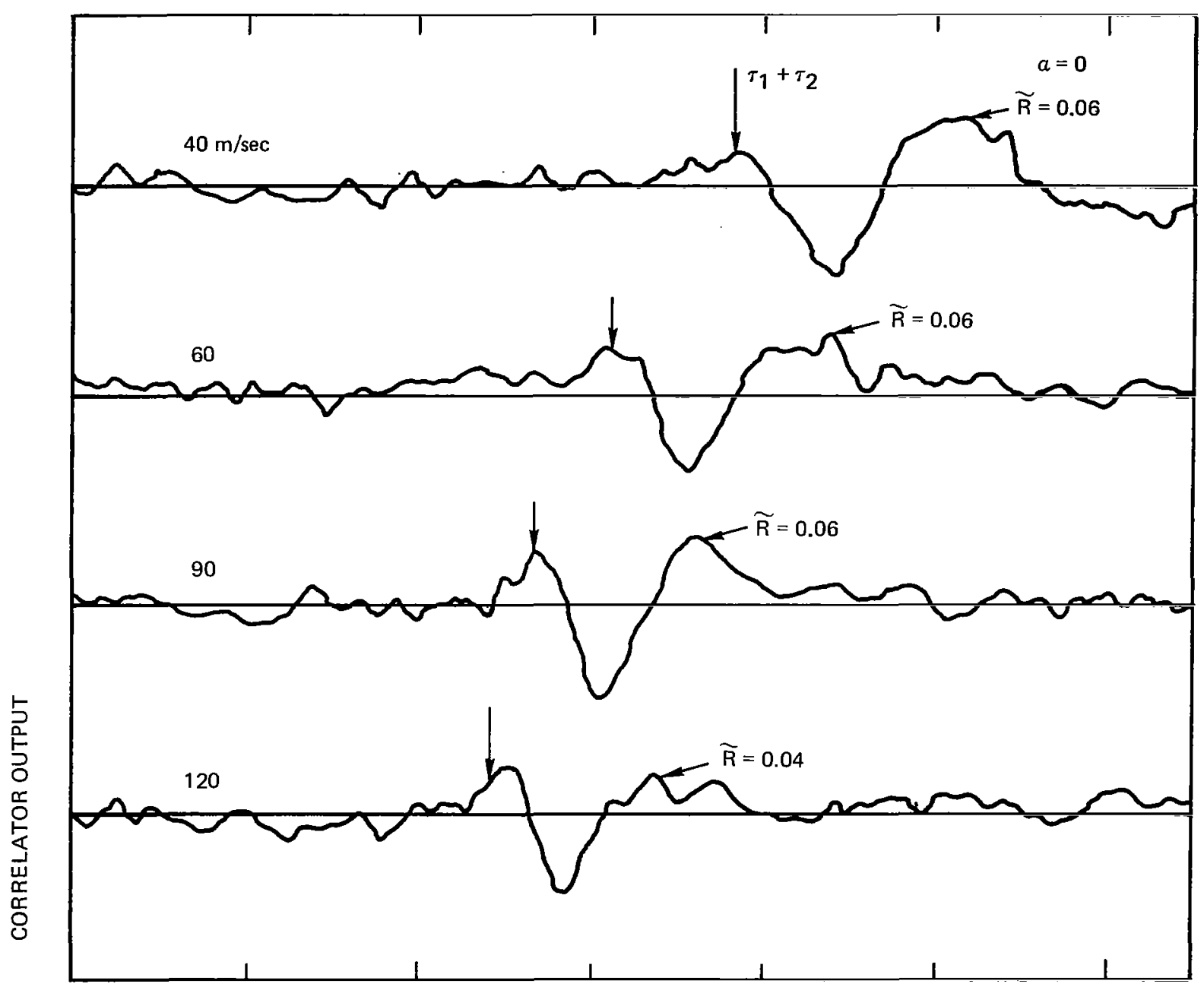

(a) VERTICAL COMPONENT

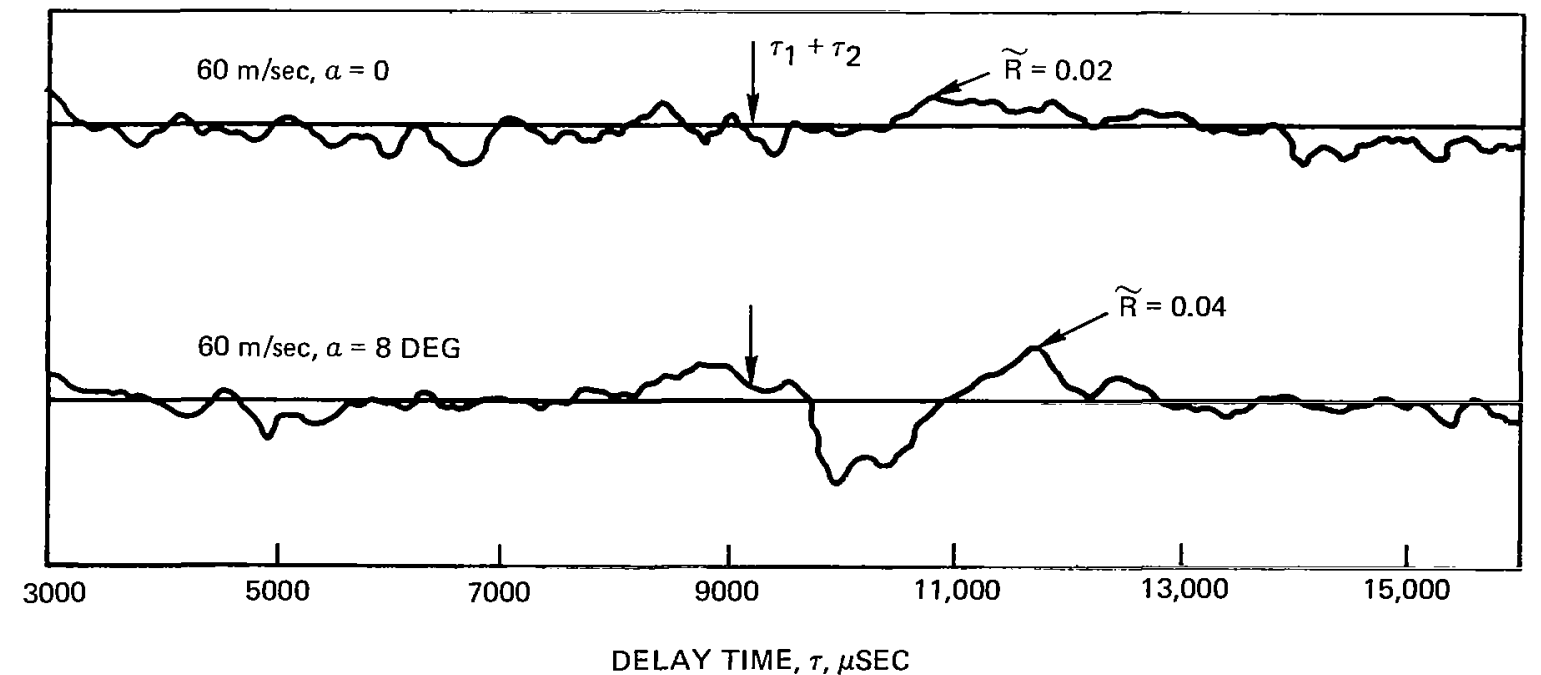

(b) AXIAL COMPONENT

FIGURE 25, - HOT WIRE FAR-FIELD CROSS-CORRELATION FUNCTIONS 


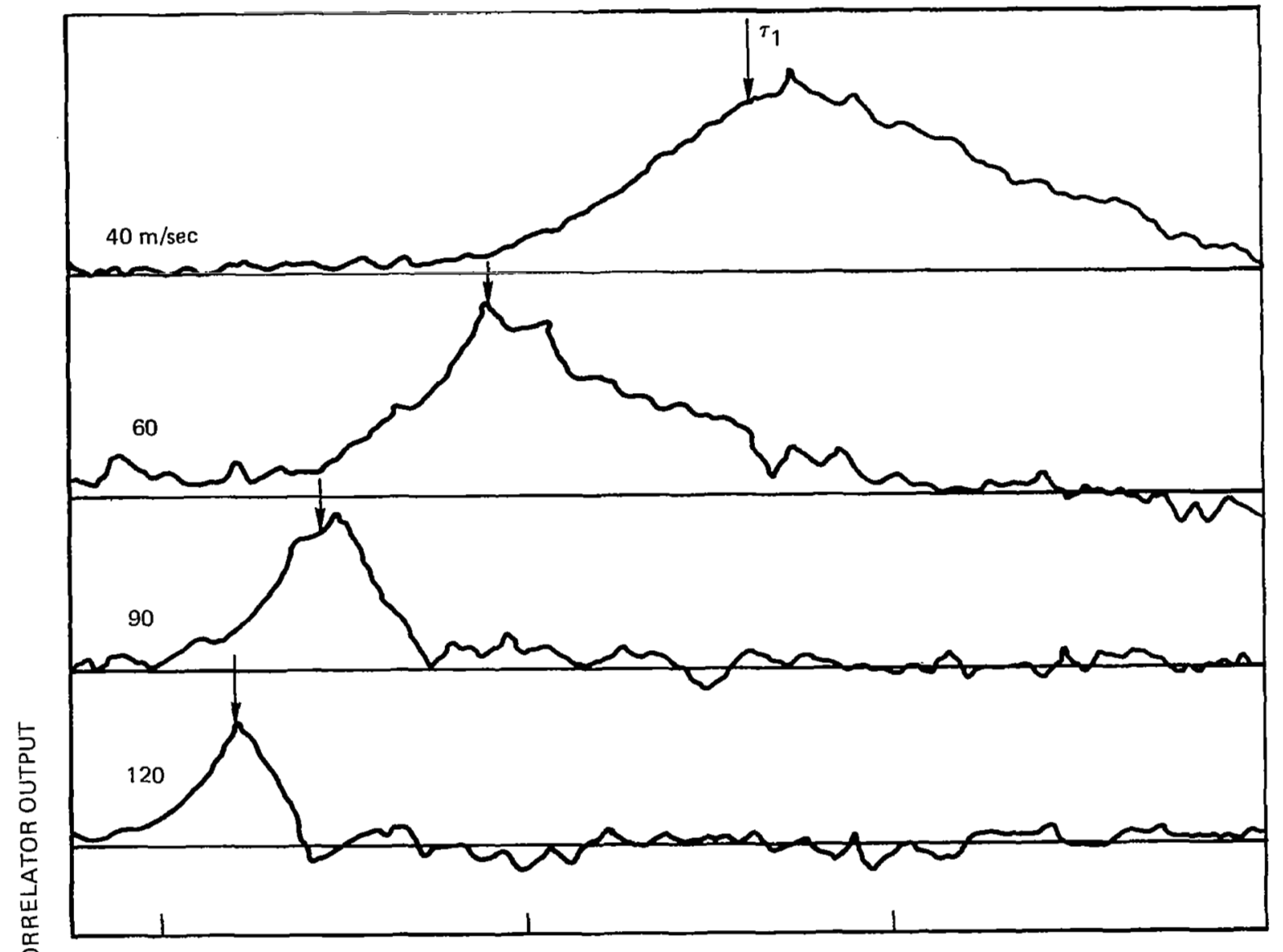

(a) $15 \%$ CHORD

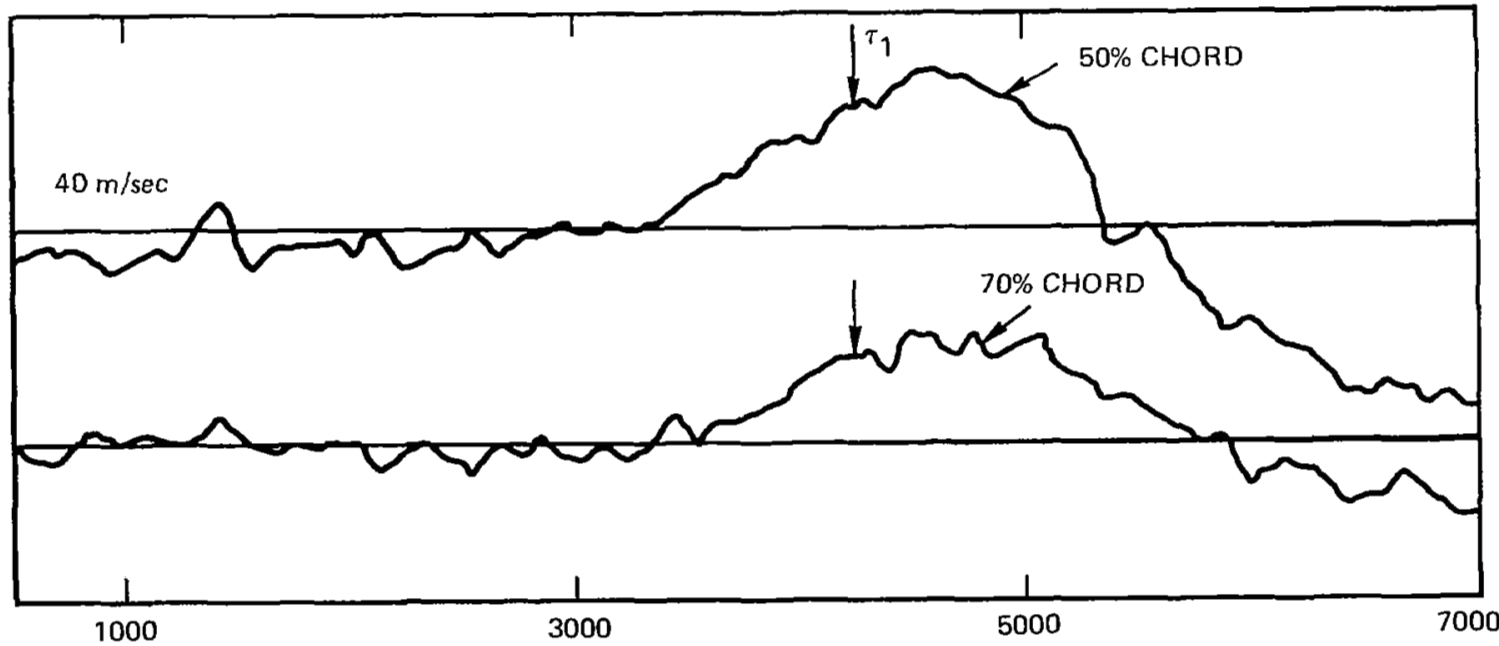

DELAY TIME $, \tau, \mu$ SEC

(b) $50 \%$ and $\mathbf{7 0} \%$ CHORD

FIGURE 26: - HOT WIRE - SURFACE PRESSURE CROSS-CORRELATION FUNCTIONS 

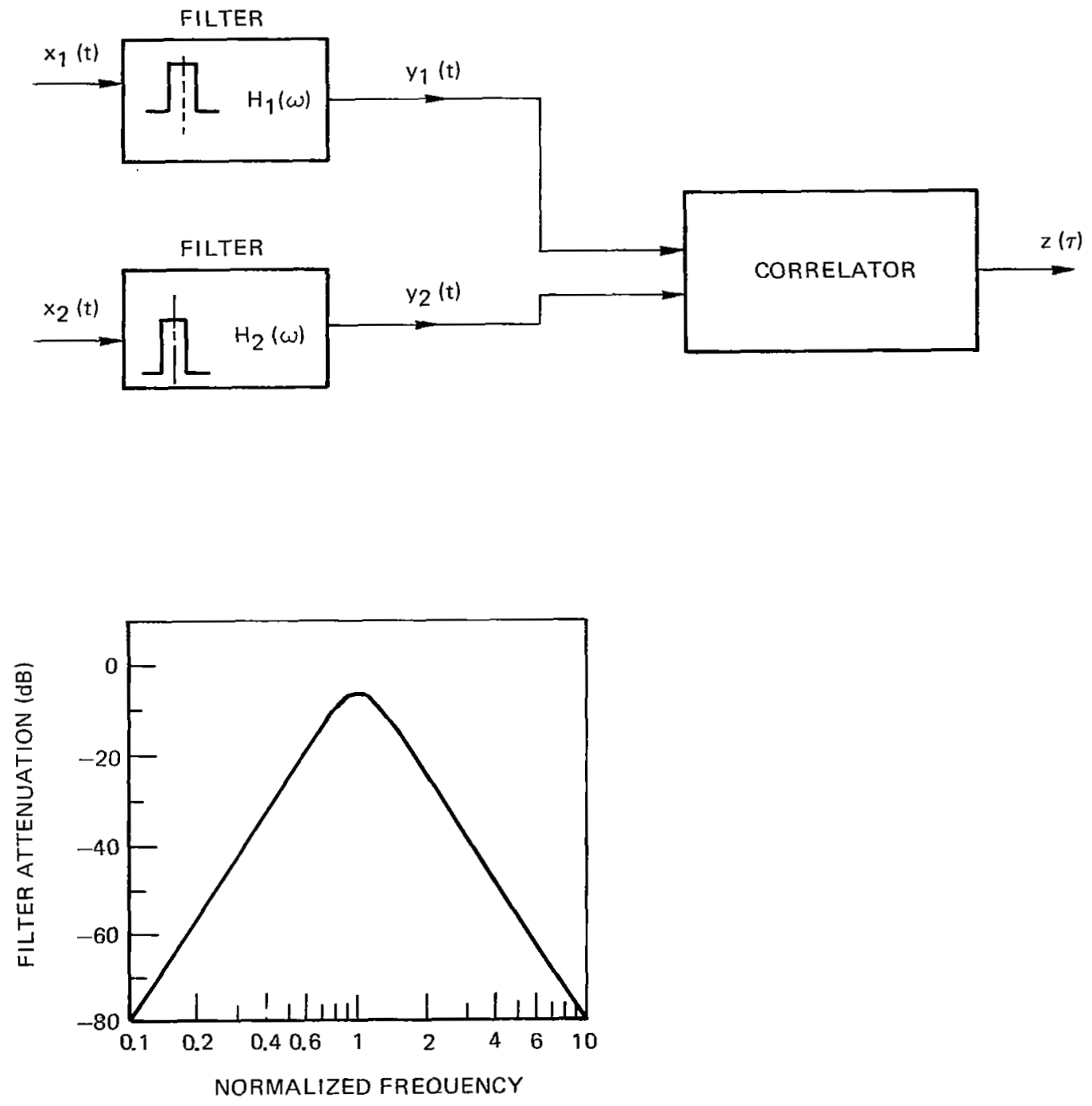

FIGURE 27. - ARRANGEMENT FOR CROSS-PSD DETERMINATION 